\title{
Superfícies compactas de tipo espaço com curvatura média constante no $\mathbb{L}^{3}$
}

Fernanda Ester Camillo Camargo

DISSERTAÇÃO APRESENTADA

AO

INSTITUTO DE MATEMÁTICA E ESTATÍSTICA

DA

UNIVERSIDADE DE SÃO PAULO

PARA

OBTENÇÃO DO GRAU DE MESTRE

EM

MATEMÁTICA PURA

Orientadora: Prof. Dra. Cláudia Cueva Cândido

Durante a elaboração deste trabalho a autora recebeu auxilio financeiro do CNPq.

São Paulo, junho de 2002. 


\section{Agradecimentos}

Obrigada à professora, orientadora e amiga Cláudia Cueva Cândido, pelos conhecimentos, dedicação, interesse, apoio e confiança em mim depositados. Eu não teria conseguido sem você. Espero que possamos trabalhar juntas por muito tempo.

Obrigada à professora Rosa Maria Barreiro Chaves, pela atenção concedida desde os meus tempos de graduação, pela participação em meus seminários e pela amizade.

Obrigada a todos os amigos que foram tão presentes no decorrer do curso.

Obrigada aos professores que, direta ou indiretamente, foram importantes para a concretização desse trabalho.

A todos vocês, todo o meu carinho e a esperança de que ainda possamos fazer muito juntos. 


\section{Resumo}

Neste trabalho, provamos alguns resultados de unicidade para as superfícies compactas de tipo espaço com curvatura média constante no espaço de Lorentz-Minkowski, usando fórmulas integrais e o princípio do máximo de Hopf. Como um resultado, provamos que as únicas tais superfícies limitadas por um círculo são os discos planares (quando $H=0$ ) e as calotas hiperbólicas (quando $H \neq 0$ ). Também obtemos um resultado análogo para o caso de dimensão $n$. 


\section{Abstract}

In this work, we prove some results of uniqueness for compact spacelike surfaces with constant mean curvature in the Lorentz-Minkowski 3-space, using integral formulas and the Hopf maximum principle. As a result, we prove that the only such surfaces bounded by a circle are the planar discs (when $H=0$ ) and the hyperbolic caps (when $H \neq 0$ ). We also obtain a analogue result for the $n$-dimensional case. 


\section{Sumário}

Lista de Figuras

xiv

Introdução

1 Preliminares $\quad 7$

1.1 A geometria dos vetores no espaço de Lorentz-Minkowski . . . . . . . 7

1.2 Geometria Semi-Riemanniana . . . . . . . . . . . . . . . . . 10

1.3 Superfícies compactas de tipo espaço . . . . . . . . . . . . . . 12

1.4 Alguns resultados de Análise . . . . . . . . . . . . . . . . 18

1.5 Geometria Diferencial Complexa . . . . . . . . . . . . . 18

1.6 Algumas notas sobre curvas no $\mathbb{R}^{3} \ldots \ldots \ldots \ldots$. . . . . . . . 22

2 Fórmulas Integrais $\quad 25$

2.1 A orientação induzida de $\partial \Sigma \ldots \ldots \ldots \ldots$

2.2 A primeira fórmula integral . . . . . . . . . . . . . . . 27

2.3 A segunda fórmula integral . . . . . . . . . . . . . . . . . 28

2.4 A terceira fórmula integral . . . . . . . . . . . . . . . 30

2.5 Fórmula do fluxo . . . . . . . . . . . . . . . . . . . . . . . . . . 32

2.6 Conseqüências da fórmula do fluxo para bordo planar . . . . . . . . . 33

2.6 .1 Uma desigualdade integral . . . . . . . . . . . . . 35 
2.7 O primeiro resultado de unicidade . . . . . . . . . . . . . . . 38

3 O Princípio do Máximo 43

3.1 Gráficos de tipo espaço . . . . . . . . . . . . . . . . . 43

3.2 A equação da curvatura média . . . . . . . . . . . . . . . . . 48

3.3 Operadores Quasilineares . . . . . . . . . . . . . . 50

3.4 O Princípio do Máximo de Hopf . . . . . . . . . . . . . . . . 52

3.5 O segundo resultado de unicidade . . . . . . . . . . . . . 53

3.6 O Princípio da Tangência . . . . . . . . . . . . . . . 57

4 Unicidade para bordo não conexo $\quad 61$

4.1 O Método de Reflexão de Alexandrov . . . . . . . . . . . . . . . . 61

4.2 Isometrias . . . . . . . . . . . . . . . . 67

4.3 Superfícies de revolução de tipo espaço . . . . . . . . . . . . . . 69

4.4 O terceiro resultado de unicidade . . . . . . . . . . . . . . 71

5 A generalização do primeiro resultado de unicidade $\quad 73$

5.1 Hipersuperfícies compactas de tipo espaço . . . . . . . . . . . . 73

5.2 Fórmula do Fluxo . . . . . . . . . . . . . . . . . 75

5.3 Conseqüências da fórmula do fluxo para bordo hiperplanar . . . . . . 78

5.4 Uma desigualdade integral . . . . . . . . . . . . . . . . 79

$\begin{array}{lc}\text { Referências Bibliográficas } & 83\end{array}$ 


\section{Lista de Figuras}

1.1 Representação dos vetores no $\mathbb{L}^{3} \ldots \ldots \ldots \ldots$. . . . . . . . 8

1.2 A aplicação de Gauss futura . . . . . . . . . . . . . . . . . . . . . 14

1.3 Curvatura com sinal . . . . . . . . . . . . . . . . . . 23

2.1 Representação da superfície imersa no $\mathbb{L}^{3} \ldots \ldots$. . . . . . . . . 25

2.2 Orientação de $\partial \Sigma \ldots \ldots \ldots$. . . . . . . . . . . . . . . . . . . 27

2.3 Representação de $\partial \Sigma$ planar . . . . . . . . . . . . . . . . . . 33

2.4 Representação das curvaturas normal e geodésica . . . . . . . . . . 37

3.1 Representação de um gráfico . . . . . . . . . . . . . . . . . . . 43

3.2 Gráfico $x \circ F \ldots \ldots \ldots$

3.3 Construção de $F \ldots \ldots \ldots \ldots$

3.4 Domínios $\Omega$ e $\Omega^{\prime} \ldots \ldots \ldots \ldots \ldots$. . . . . . . . . . . . 47

3.5 Representação de $\Sigma_{1}$ e $\Sigma_{2} \ldots \ldots \ldots \ldots \ldots \ldots$

3.6 Caso geral para o teorema $3.5 .2 \ldots \ldots \ldots 6$

3.7 O Princípio da tangência . . . . . . . . . . . . . . . . 57

3.8 Superfície compacta máxima com bordo planar . . . . . . . . . . 59

4.1 Anel determinado por $\Gamma_{1}$ e $\pi\left(\Gamma_{2}\right) \ldots \ldots \ldots 62$

4.2 Superfície com bordo $\Gamma_{1} \cup \Gamma_{2} \ldots \ldots \ldots \ldots$. . . . . . . . . . . 64 
4.3 Método de reflexão de Alexandrov para $\Sigma \ldots \ldots . \ldots . \ldots 65$ 


\section{Introdução}

Neste trabalho fazemos um estudo das superfícies compactas imersas no espaço tridimensional de Lorentz-Minkowski que são de tipo espaço (isto é, a métrica induzida. pela imersão é Riemanniana) e possuem curvatura média constante.

A justificativa para a escolha deste tema é que tais superfícies representam um papel importante tanto do ponto de vista físico quanto do ponto de vista matemático. No primeiro caso, elas aparecem em diferentes problemas na relatividade geral. Por exemplo, as superfícies (ou mais geralmente, as hipersuperfícies) de tipo espaço com curvatura média constante não nula são convenientes para se estudar a propagação das ondas gravitacionais. No segundo caso, seu interesse é motivado pelo fato dessas superfícies exibirem boas propriedades do tipo Bernstein, que são resultados em que, sob certas hipóteses, pode-se concluir que as superfícies são planos [11], além de serem soluções para um problema variacional, uma vez que são pontos críticos do funcional área para variações que deixam constante uma certa função volume. É claro que ainda temos a oportunidade de conhecer o espaço de Lorentz-Minkowski, buscando as analogias e as diferenças entre ele e o espaço Euclideano.

A Geometria de Lorentz é o modelo matemático usado na relatividade geral. O seu maior interesse deveu-se ao progresso na teoria de causalidade, na teoria de singularidades e buracos negros na relatividade geral, influenciado pelas pesquisas de S. Hawking, por volta de 1970. (Algumas referências desse assunto podem ser consultadas em [8])

A motivação física para se estudar variedades Lorentzianas é a hipótese de que um campo gravitacional pode ser efetivamente modelado por alguma métrica Lorentziana $g$ definida sobre uma variedade $M$. Em particular, quando a dimensão dessa variedade é 4, temos as equações de Einstein que ligam a Geometria (em termos de curvatura) 
à. Física (em termos de massa e energia). Estas são equações diferenciais parciais relacionando o tensor métrico e suas primeiras derivadas ao tensor momento-energia $T$. Isso é importante na relatividade geral porque assume-se que o comportamento do universo é como um fluido perfeito (grosseiramente falando, com viscosidade zero) nos modelos cosmológicos padrões. E o tensor momento-energia para o caso de um fluido perfeito tem uma forma simples.

Uma vez que toda variedade que admite uma métrica Lorentziana, admite várias métricas desse tipo, é necessário decidir qual variedade e qual métrica Lorentziana sobre ela devem ser usadas para se modelar um problema gravitacional dado. As equações de Einstein relacionam o tensor métrico $g$, a curvatura de Ricci Ric e a curvatura escalar $R$ ao tensor momento energia $T$ e podem ser escritas invariavelmente como

$$
R i c-\frac{1}{2} R g+\Lambda g=8 \pi T
$$

onde $\Lambda$ é uma constante conhecida como a constante cosmológica. O fator constante $8 \pi$ está presente por propósitos de escala.

Em coordenadas

$$
R_{i j}-\frac{1}{2} R g_{i j}+\Lambda g_{i j}=8 \pi T_{i j} \quad 1 \leq i, j \leq 4
$$

A curvatura de Ricci e a curvatura escalar envolvem a primeira e a segunda derivadas parciais dos componentes $g_{i j}$ da métrica. Conseqüentemente, as equações de Einstein representam equações diferenciais parciais (não-lineares) na métrica e suas duas primeiras derivadas.

As equações de Einstein não determinam a métrica sobre $M$ sem condições suficientes para o bordo.

A. Lichnerowicz, em seu clássico artigo L'integration des equations de la gravitation relativiste et le probleme des $n$ corps mostrou que o problema de Cauchy da equação de Einstein com condições sobre uma hipersuperfície máxima se reduz a um sistema linear diferencial de primeira ordem e a uma equação diferencial de segunda ordem elíptica não-linear.

Abordagens mais profundas para o problema de existência e unicidade das soluções para as equações de Einstein podem ser encontradas, por exemplo, em trabalhos de 
S. Hawking e Y. Choquet-Bruhat.

A técnica usada em nosso estudo é baseada na investigação da influência do bordo na forma da superfície. Os resultados apresentados foram provados por L. J. Alías, J. A. Pastor e R. López e podem ser encontrados em [4], que é a principal referência utilizada.

Apresentamos alguns resultados de unicidade para tais superfícies a partir de duas abordagens diferentes. A primeira abordagem é baseada em algumas fórmulas integrais para superfícies compactas de tipo espaço no $\mathbb{L}^{3}$, entre elas, a Fórmula do Fluxo, a qual, grosseiramente falando, diz que uma superfície compacta de tipo espaço com curvatura média constante imersa no $\mathbb{L}^{3}$ está em equilíbrio com respeito à tensão da superfície e às forças de pressão externas. Esta fórmula também é utilizada, numa outra formulação, a fim de se encontrar resultados de unicidade para as superfícies com curvatura média constante imersas no espaço Euclideano de dimensão 3. Por exemplo, em [19], R. López e S. Montiel estudam condições para que um disco imerso com curvatura média constante $H$ não nula limitado por um círculo seja uma calota esférica a partir.de certas restrições para a área desse disco e para a constante $H$.

Quanto às outras fórmulas integrais obtidas, é necessário, em algum momento, considerarmos a superfície como uma variedade complexa e tomarmos nela uma estrutura quase complexa $J$, que é um campo tensorial tal que $J^{2}=-I d$. Apesar de nosso trabalho não tratar diretamente deste assunto, achamos importante situar a superfície nesse ambiente já que, em outros trabalhos envolvendo o espaço de LorentzMinkowski e as superfícies compactas de tipo espaço com curvatura média constante, esse resultado é bastante utilizado. Também decorrem algumas conseqüências para o caso em que o bordo é uma curva de Jordan contida num plano de tipo espaço no $\mathbb{L}^{3}$.

A segunda abordagem utiliza o fato de que uma superfície de tipo espaço com curvatura média constante e bordo que se projeta sobre uma curva de Jordan num plano de tipo espaço pode ser vista como um gráfico de tipo espaço e, portanto, satisfaz uma equação elíptica não-linear, que é a equação da curvatura média, para a qual aplicamos o Princípio do Máximo de Hopf. Ainda com esse princípio e com o Método de Reflexão de Alexandrov, obtemos um resultado de unicidade para as superfícies compactas de tipo espaço com curvatura média constante limitadas por 
dois círculos concêntricos localizados em planos paralelos.

O trabalho apresenta-se com a seguinte divisão:

No primeiro capítulo, definimos os principais conceitos da teoria da Geometria Semi-Riemanniana e, em especial, do espaço de Lorentz-Minkowski, tais como variedades, métrica, conexões e equações fundamentais. Apresentamos algumas propriedades sobre curvas, que serão necessárias por serem os bordos das superfícies tratadas, mostramos um pouco sobre a Geometria Diferencial Complexa, além de expormos resultados de Análise que são essenciais para a obtenção das fórmulas e nas demonstrações de alguns teoremas.

No segundo capítulo, desenvolvemos as fórmulas integrais, as quais são essenciais para a prova do primeiro resultado de unicidade que é o seguinte:

As únicas superficies compactas de tipo espaço com curvatura média constante imersas no $\mathbb{L}^{3}$ e bordo circular são os discos planares e as calotas hiperbólicas.

O terceiro capítulo é destinado a um breve estudo das equações elípticas e do Princípio do Máximo de Hopf. Com ele, enunciamos e provamos o seguinte teorema de unicidade:

Sejam $\Sigma_{1}$ e $\Sigma_{2}$ duas superfícies compactas de tipo espaço com curvatura média constante imersas no $\mathbb{L}^{3}$ limitadas por uma curva que se projeta sobre uma curva de Jordan contida num plano de tipo espaço. Se $\Sigma_{1}$ e $\Sigma_{2}$ têm a mesma curvatura média para uma orientação comum, então $\Sigma_{1}=\Sigma_{2}$.

Observamos que, com este resultado, obtemos uma prova alternativa para o primeiro resultado de unicidade.

Como anexo deste capítulo, apresentamos o Princípio da Tangência para superfícies de tipo espaço com curvatura média constante que aparece em [4], [6]. [17] e [18].

Para o quarto capítulo, reservamos o estudo do caso em que o bordo da superfície não é conexo. Mais precisamente, quando o bordo consiste de duas curvas de Jordan planas contidas em planos de tipo espaço paralelos. Em particular, prova-se que:

Se $\Sigma$ é uma superfície compacta de tipo espaço máxima imersa no $\mathbb{L}^{3}$ limitada por dois círculos concêntricos em planos paralelos então $\Sigma$ é uma superfície de revolução. 
Neste capítulo, fazemos também um breve estudo das isometrias para podermos tratar das superfícies de revolução.

No quinto capítulo, exibimos a generalização do primeiro resultado de unicidade, encontrado em [5]. Vale ressaltar que as técnicas aqui utilizadas são análogas às que aparecem no Capítulo 2. Obtém-se, neste capítulo, uma fórmula do fluxo e uma desigualdade integral, para se provar que

As únicas hipersuperfícies compactas de tipo espaço imersas no $\mathbb{L}^{n+1}$ com curvatura média constante $H$ e bordo esférico são as bolas hiperplanares e as calotas hiperbólicas.

A demonstração apresentada pelos autores nessa generalização, em que a dimensão da superfície pode ser ímpar, é diferente da feita para a dimensão 2 , caso em que foi possível munir a superfície com uma estrutura quase complexa. Tal demonstração poderia ter sido utilizada na situação bi-dimensional, entretanto, escolhemos seguir fielmente a solução encontrada em [4], por ela ser a principal referência deste trabalho. 


\section{Capítulo 1}

\section{Preliminares}

Neste capítulo, estão os principais conceitos, notações e teoremas utilizados no decorrer do trabalho. Eles podem ser encontrados com mais detalhes em [11], [22] e [23].

Denotamos por $\mathbb{L}^{3}=\left(\mathbb{R}^{3},\langle\rangle,\right)$ o espaço tridimensional de Lorentz-Minkowski, onde $\langle\rangle=,\left(d x_{1}\right)^{2}+\left(d x_{2}\right)^{2}-\left(d x_{3}\right)^{2}$ é a métrica Lorentziana e $\left(x_{1}, \dot{x}_{2}, x_{3}\right)$ são as coordenadas canônicas no $\mathbb{R}^{3}$.

\subsection{A geometria dos vetores no espaço de Lorentz- Minkowski}

Um vetor $v \in \mathbb{L}^{3}$ é dito

$$
\begin{gathered}
\text { de tipo espaço, se }\langle v, v\rangle>0 \text { ou } v=0 . \\
\text { de tipo luz, se }\langle v, v\rangle=0 \text { e } v \neq 0 \\
\text { de tipo tempo, se }\langle v, v\rangle<0 .
\end{gathered}
$$

Cada uma destas categorias é chamada de caráter causal. 
Da mesma forma, se $\Pi \subset \mathbb{L}^{3}$ é um plano, então

- II é dito de tipo espaço se os seus vetores são de tipo espaço.

- II é dito de tipo tempo se possui um vetor de tipo tempo.

- II é dito de tipo luz se possui um vetor de tipo luz e não possui vetor de tipo tempo.

Chamamos de cone de luz o conjunto que contém os vetores $v \in \mathbb{L}^{3}$ tais que $\langle v, v\rangle=0 \operatorname{com} v \neq 0$.

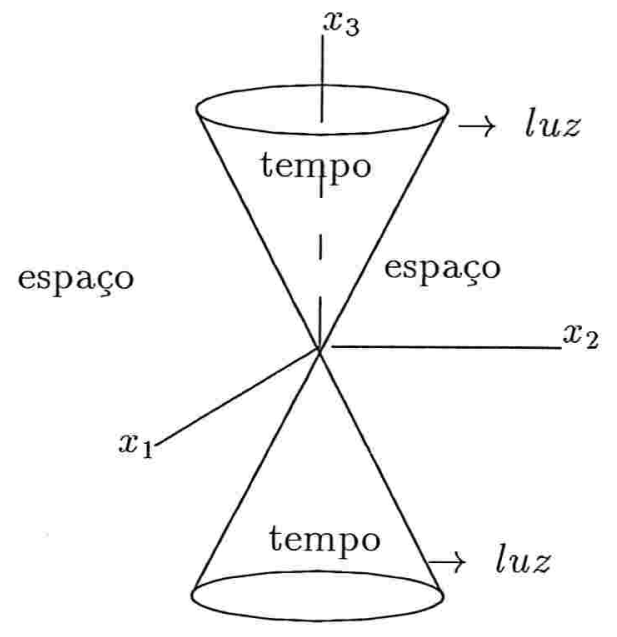

Figura 1.1: Representação dos vetores no $\mathbb{L}^{3}$

Também podemos definir um produto vetorial no $\mathbb{L}^{3}$ da seguinte forma:

Definição 1.1.1. O produto vetorial no $\mathbb{L}^{3}$ de dois vetores $v, w \in \mathbb{L}^{3}$ é o único vetor $v \wedge w \in \mathbb{L}^{3}$ tal que $\langle v \wedge w, u\rangle=\operatorname{det}(v, w, u) \forall u \in \mathbb{L}^{3}$.

Se $v=\left(v_{1}, v_{2}, v_{3}\right)$ e $w=\left(w_{1}, w_{2}, w_{3}\right)$ então $v \wedge w$ é dado em coordenadas pela expressão

$$
v \wedge w=\left|\begin{array}{ccc}
\vec{i} & \vec{j} & \vec{k} \\
v_{1} & v_{2} & v_{3} \\
w_{1} & w_{2} & w_{3}
\end{array}\right|=\left(v_{2} w_{3}-v_{3} w_{2}, v_{3} w_{1}-v_{1} w_{3}, v_{2} w_{1}-v_{1} w_{2}\right)
$$




\section{Propriedades do Produto Vetorial:}

$\operatorname{Sejam} u, v \in \mathbb{L}^{3}$

1. $u \wedge v=0 \Leftrightarrow u, v$ são linearmente dependentes.

2. $u \wedge v=-v \wedge u$.

3. $\langle u \wedge v, u \wedge v\rangle=\langle u, v\rangle^{2}-\langle u, u\rangle\langle v, v\rangle$.

4. Se $u$ ou $v$ é de tipo tempo, $u \wedge v$ é de tipo espaço.

5. Se $u$ e $v$ são de tipo espaço, $u \wedge v$ é de tipo tempo.

6. $u \wedge v$ é de tipo luz $\Leftrightarrow u \wedge v \neq 0$ e pertence ao plano gerado por $u$ e $v$.

Como conseqüência da Definição 1.1 .1 vale que se $u$ e $v$ são ortonormais, o conjunto $\{u, v, u \wedge v\}$ forma uma base ortonormal do $\mathbb{L}^{3}$.

\section{Cones de tipo tempo}

Seja $T$ o conjunto de todos os vetores de tipo tempo no $\mathbb{L}^{3}$.

Definição 1.1.2. Se $u \in T, C(u)=\{v \in T:\langle u, v\rangle\langle 0\}$ é chamado o cone de tipo tempo do $\mathbb{L}^{3}$ que contém $u$.

Lema 1.1.3. Dois vetores de tipo tempo $v, w$ no $\mathbb{L}^{3}$ estão no mesmo cone de tipo tempo se e somente se $\langle v, w\rangle<0$.

Proposição 1.1.4 (Desigualdade de Cauchy-Schwarz invertida). Sejam v, w vetores de tipo tempo no $\mathbb{L}^{3}$. Então

$$
|\langle v, w\rangle| \geq|v||w| \text { e a igualdade vale se e somente se } v \text { e } w \text { são colineares. }
$$

Lema 1.1.5. Se $z$ é um vetor de tipo tempo no $\mathbb{L}^{3}$ então o subespaço $z^{\perp}$ é de tipo espaço.

Definição 1.1.6. Dois vetores $v, w \in \mathbb{L}^{3}$ são ortogonais se $\langle v, w\rangle=0$. Um vetor $v$ é dito unitário se $\langle v, v\rangle=1$ ou $\langle v, v\rangle=-1$. 


\subsection{Geometria Semi-Riemanniana}

Nesta seção, definimos variedades semi-Riemannianas a partir dos conceitos de tensores, além de estabelecermos notações e propriedades da derivação de campos de vetores na direção de campos numa variedade.

\section{Campos de tensores}

Sejam $V$ um módulo sobre um anel $K$ e $V^{*}$ o conjunto de todas as funções $K$ lineares definidas em $V$ a valores em $K$, que também é um módulo com as operações usuais de adição de funções e multiplicação por escalar de $K$. $V^{*}$ é chamado o módulo dual de $V$.

Definição 1.2.1. Para $r \geq 0, s \geq 0$ não simultaneamente nulos, dizemos que uma função $K$-multilinear $T:\left(V^{*}\right)^{r} \times V^{s} \rightarrow K$ é chamada um tensor do tipo $(r, s)$ sobre $V$.

Seja $M$ uma variedade diferenciável e denotemos por $\mathcal{F}(M)$ o conjunto de funções diferenciáveis a valores reais em $M$ e $\mathcal{X}(\mathrm{M})$ o conjunto de todos os campos vetoriais diferenciáveis em $M$.

Definição 1.2.2. Um campo de tensores (ou campo tensorial) $T$ em uma variedade $M$ é um tensor do tipo $(r, s)$

$$
T: \mathcal{X}^{*}(M)^{r} \times \mathcal{X}(M)^{s} \rightarrow \mathcal{F}(M)
$$

T leva $r$ 1-formas e s campos vetoriais numa função $f$, isto é, $T\left(\theta_{1}, \ldots, \theta_{r}, X_{1}, \ldots, X_{s}\right)=$ $f$, onde $\theta_{i}$ são 1 -formas e $X_{j}$ são campos vetoriais.

Definição 1.2.3. Um tensor métrico $g$ em uma variedade diferenciável $M$ é um campo tensorial do tipo $(0,2)$ simétrico, não degenerado, de índice constante (que é o maior inteiro que é a dimensão de um subespaço $\mathcal{W} \subset \mathcal{X}(M)$ em que $\left.g_{p}\right|_{\mathcal{W}}$ é definida negativa, isto é, se $v \neq 0$ então $g_{p}(v, v)<0$ para $\left.p \in M\right)$.

Observação 1.2.4. Em outras palavras, $g$ associa diferenciavelmente a cada ponto $p \in M$ um produto escalar $g_{p}$ no espaço tangente $T_{p} M$ e o índice de $g_{p}$ é o mesmo para todo $p$. 
Definição 1.2.5. Uma variedade semi-Riemanniana $M$ é uma variedade diferenciável com um tensor métrico $g$.

Definição 1.2.6. Uma derivação tensorial $\mathcal{D}$ sobre uma variedade semi-Riemanniana $M$ é um conjunto de funções $\mathbb{R}$-lineares

$$
\mathcal{D}: \tau_{s}^{r}(M) \rightarrow \tau_{s}^{r}(M) \quad(r \geq 0, s \geq 0)
$$

onde $\tau_{s}^{r}$ é o conjunto de todos os campos tensoriais sobre $M$ do tipo $(r, s)$, tais que para todos tensores $A, B$

(1) $\mathcal{D}(A \otimes B)=\mathcal{D} A \otimes B+A \otimes \mathcal{D} B$

(2) $\mathcal{D}(C A)=C(\mathcal{D} A)$ para toda contração $C$

Definição 1.2.7. Uma contração $C$ é uma aplicação $C: \tau_{s}^{r}(M) \rightarrow \tau_{s-1}^{r-1}$ que transforma tensores do tipo $(r, s)$ em tensores do tipo $(r-1, s-1)$.

Observação 1.2.8. Todo tensor $T$ do tipo $(0, s)$ pode ser visto como um tensor $(1, s)$. Isso é feito considerando (no lugar de T) um tensor $\bar{T}: \mathcal{X}^{*}(M) \times \mathcal{X}(M)^{s} \rightarrow \mathcal{F}(M)$ definido por

$$
\bar{T}\left(\theta, X_{1}, \ldots, X_{s}\right)=\theta\left(T\left(X_{1}, \ldots, X_{s}\right)\right) \forall \theta \in \mathcal{X}^{*}(M), X_{i} \in \mathcal{X}(M)
$$

Proposição 1.2.9 (A regra do produto). Se Té um tensor do tipo $(r, s)$ então

$$
\begin{gathered}
(\mathcal{D} T)\left(\theta_{1}, \ldots, \theta_{r}, X_{1}, \ldots, X_{s}\right)=\mathcal{D}\left(T\left(\theta_{1}, \ldots, \theta_{r}, X_{1}, \ldots, X_{s}\right)\right)+ \\
\quad-\sum_{i=1}^{r} T\left(\theta_{1}, \ldots, \theta_{i-1}, \mathcal{D} \theta_{i}, \theta_{i+1}, \ldots, \theta_{r}, X_{1}, \ldots, X_{s}\right)+ \\
-\sum_{j=1}^{s} T\left(\theta_{1}, \ldots, \theta_{r}, X_{1}, \ldots, X_{j-1}, \mathcal{D} X_{j}, X_{j+1}, \ldots, X_{s}\right)
\end{gathered}
$$

Definição 1.2.10. Seja T um campo tensorial do tipo $(r, s)$ sobre uma variedade $M$. A diferencial covariante de $T$ é um tensor DT do tipo $(r, s+1)$ dado por

$$
\operatorname{DT}\left(\theta_{1}, \ldots, \theta_{r}, X_{1}, \ldots, X_{s}, X\right)=\left(D_{X} T\right)\left(\theta_{1}, \ldots, \theta_{r}, X_{1}, \ldots, X_{s}\right)
$$

para cada $\theta_{i} \in \mathcal{X}(M), X, X_{i} \in \mathcal{X}(M)$, onde $D$ é a derivada covariante em $M$. 


\section{A Conexão Levi-Civita}

Uma conexão Levi-Civita em $M$ é um tensor $(0,2) \nabla: \mathcal{X}(M) \times \mathcal{X}(M) \rightarrow \mathcal{X}(M)$ tal que para quaisquer campos $X, Y, Z \in \mathcal{X}(M)$, e para quaisquer funções $f, g \in \mathcal{F}(M)$ valem

1. $\nabla_{X+Y} Z=\nabla_{X} Z+\nabla_{Y} Z$

2. $\nabla_{X}(Y+Z)=\nabla_{X} Y+\nabla_{X} Z$

3. $\nabla_{f X} Y=f \nabla_{X} Y$

4. $\nabla_{X}(f Y)=X(f) Y+f \nabla_{X} Y$

5. $\nabla_{X} Y-\nabla_{Y} X=[X, Y]$ onde $[X, Y](f)=X(Y f)-Y(X f)$ (simetria)

6. $X\langle Y, Z\rangle=\left\langle\nabla_{X} Y, Z\right\rangle+\left\langle Y, \nabla_{X} Z\right\rangle$ (compatibilidade com a métrica)

As condições (1) a (4) definem o que chamamos de conexão afim. Já as condições (5) e (6) são as essenciais para podermos construir uma conexão Levi-Civita numa variedade semi-Riemanniana.

Teorema 1.2.11. Se $M$ é uma variedade semi-Riemanniana, existe uma única conexão Levi-Civita em $M$.

\subsection{Superfícies compactas de tipo espaço}

Denotemos por $\Sigma$ uma superfície diferenciável, isto é, uma variedade diferenciável de dimensão 2.

Definição 1.3.1. Uma imersão $x: \Sigma \rightarrow \mathbb{L}^{3}$ é uma aplicação diferenciável tal que $d x_{p}: T_{p} \Sigma \rightarrow T_{p} \Sigma$ é injetora $\forall p \in \Sigma$.

São equivalentes:

(a) A aplicação $d x_{p}$ é 1-1;

(b) O Jacobiano de $d x_{p}$ tem posto 2 relativo a uma escolha de sistemas coordenados 
(conseqüentemente a toda escolha);

(c) Se $\left(y_{1}, y_{2}, y_{3}\right)$ é um sistema de coordenadas no $\mathbb{L}^{3}$ em $x(p)$, então há inteiros $1 \leq i_{1} \leq i_{2} \leq i_{3} \leq 3$ tais que as funções $\left\{y^{i_{1}} \circ x, y^{i_{2}} \circ x, y^{i_{3}} \circ x\right\}$ formam um sistema de coordenadas numa vizinhança de $p$ em $\Sigma$.

Definição 1.3.2. Um mergulho de $\Sigma$ no $\mathbb{L}^{3}$ é uma imersão injetora $X: \Sigma \rightarrow \mathbb{L}^{3}$ tal que a aplicação induzida $\Sigma \rightarrow X(\Sigma)$ é um homeomorfismo sobre o subespaço $X(\Sigma)$ do $\mathbb{L}^{3}$.

Definição 1.3.3. Uma métrica Riemanniana $\langle$,$\rangle em uma superfície \Sigma$ é um tensor métrico de índice zero (isto é, definido positivo em todo $\mathcal{X}(M)$ ) e, portanto, associa a cada $p \in \Sigma$ uma aplicação $\langle,\rangle_{p}: T_{p} \Sigma \times T_{p} \Sigma \rightarrow \mathbb{R}$ satisfazendo, para todos $u, v, w \in T_{p} \Sigma e \lambda \in \mathbb{R}$, as seguintes propriedades:

1. $\langle u, v\rangle_{p}=\langle v, u\rangle_{p}$ (simetria)

2. $\langle u+v, w\rangle_{p}=\langle u, w\rangle_{p}+\langle v, w\rangle_{p}$ $\langle\lambda u, v\rangle_{p}=\lambda\langle u, v\rangle_{p}=\langle u, \lambda v\rangle_{p}$ (bilinearidade)

3. $\langle u, u\rangle_{p} \geq 0 e\langle u, u\rangle_{p}=0 \Leftrightarrow u=0$

4. Se $X$ e $Y$ são campos diferenciáveis em um aberto $U \subset \Sigma$, então a aplicação

$$
U \subset \Sigma \rightarrow \mathbb{R}
$$

é diferenciável em $U$

$$
p \mapsto\langle X(p), Y(p)\rangle_{p}
$$

Definição 1.3.4. Dizemos que $\Sigma$ é uma superfície de tipo espaço imersa no $\mathbb{L}^{3}$ se a métrica induzida pela imersão diferenciável $x: \Sigma \rightarrow \mathbb{L}^{3}$ é Riemanniana em $\Sigma$. A métrica em $\Sigma$, também denotada por $\langle$,$\rangle , fica definida em T_{p} \Sigma \times T_{p} \Sigma$, a partir da métrica no $\mathbb{L}^{3}$, do seguinte modo

$$
\langle u, v\rangle_{p}=\left\langle d x_{p}(u), d x_{p}(v)\right\rangle_{x(p)}, \forall u, v \in T_{p} \Sigma, p \in \Sigma
$$




\section{A aplicação de Gauss futura}

Para uma superfície de tipo espaço $\Sigma$ no $\mathbb{L}^{3}$, a aplicação de Gauss $N$ de $\Sigma$ é, por definição, uma aplicação de $\Sigma$ no $\mathbb{L}^{3}$ que associa, a cada ponto $p \in \Sigma$, o ponto no $\mathbb{L}^{3}$ obtido transladando paralelamente o vetor normal unitário $n(p)$ de $\Sigma$ em para a origem do $\mathbb{L}^{3}$. Note que, como $n(p)$ é um vetor unitário de tipo tempo em $p \in \mathbb{L}^{3}$, a aplicação de Gauss $N$ é na verdade uma aplicação de $\Sigma$ no $\mathbb{H}^{2}$ onde

$$
\mathbb{H}^{2}=\left\{x \in \mathbb{L}^{3}:\langle x, x\rangle=-1, x_{3} \geq 1\right\}, x=\left(x_{1}, x_{2}, x_{3}\right) .
$$

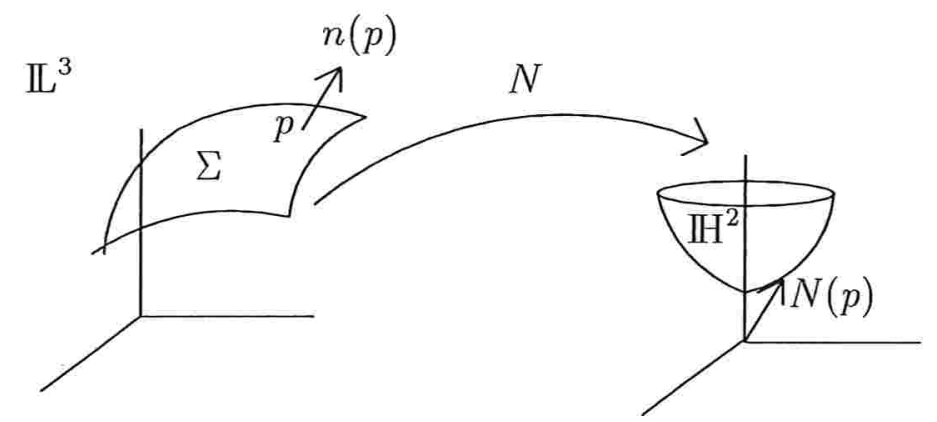

Figura 1.2: A aplicação de Gauss futura

Consideremos, a partir de agora, $\Sigma$ uma superfície de tipo espaço, conexa e imersa no $\mathbb{L}^{3}$ orientada pelo campo vetorial normal unitário de tipo tempo $N$ apontando para o futuro no $\mathbb{L}^{3}$.

\section{As equações fundamentais de $\Sigma$}

Como vimos no teorema 1.2.11, podemos definir uma conexão Levi-Civita em $\Sigma$ e o mesmo podemos fazer para o $\mathbb{L}^{3}$, tendo em vista que ele é uma variedade semiRiemanniana.

Denotemos por $\nabla^{\circ}$ a conexão Levi-Civita do $\mathbb{L}^{3}$ e $\nabla$ a conexão Levi-Civita de $\Sigma$. Além disto, denotemos por $\mathcal{X}(\Sigma)^{\perp}$ o conjunto de campos vetoriais normais a $\Sigma \mathrm{e}$ $\overline{\mathcal{X}}(\Sigma)=\mathcal{X}(\Sigma) \oplus \mathcal{X}(\Sigma)^{\perp}$. 
Definimos a conexão induzida de $\Sigma$ imersa no $\mathbb{L}^{3}$ como uma aplicação

$$
\nabla^{\circ}: \mathcal{X}(\Sigma) \times \overline{\mathcal{X}}(\Sigma) \rightarrow \overline{\mathcal{X}}(\Sigma)
$$

tal que

$$
\nabla_{X}^{\circ} Y(p):=\nabla_{\bar{X}}^{\circ} \bar{Y}(x(q))
$$

em cada vizinhança $\mathcal{U} \cap \Sigma$, onde $\bar{X}$ e $\bar{Y}$ são as extensões locais diferenciáveis de $X$ e $Y$, respectivamente, numa vizinhança coordenada $\mathcal{U}$ de $p$ no $\mathbb{L}^{3}$. Por exemplo, o campo $\bar{X} \in \mathbb{L}^{3}$ tal que $\bar{X} \circ x_{\mid \mathcal{U}}=d x \circ X_{\mathcal{U}}$ é uma extensão local de $X, \forall X \in \mathcal{X}(\Sigma)$.

Uma vez que a conexão induzida acima está intimamente relacionada com a conexão Levi-Civita de $\mathbb{L}^{3}$, usamos a mesma notação para ambas.

Se $X, Y \in \mathcal{X}(\Sigma)$, o campo $\nabla_{X}^{\circ} Y$ não está necessariamente em $\mathcal{X}(\Sigma)$. Temos, assim, a seguinte equação relacionando as conexões Levi-Civita de $\Sigma$ e do $\mathbb{L}^{3}$

$$
\nabla_{X}^{\circ} Y=\nabla_{X} Y+\left(\nabla_{X}^{\circ} Y\right)^{\perp}
$$

onde $\nabla_{X} Y$ e $\left(\nabla_{X}^{\circ} Y\right)^{\perp}$ são, respectivamente, a parte tangente e a parte normal a $\Sigma$ de $\nabla_{X}^{\circ} Y, X, Y \in \mathcal{X}(\Sigma)$.

Definimos o tensor $\sigma: \mathcal{X}(\Sigma) \times \mathcal{X}(\Sigma) \rightarrow \mathcal{X}^{\perp}(\Sigma)$ dado por

$$
\sigma(X, Y)=\left(\nabla_{X}^{\circ} Y\right)^{\perp}
$$

como o tensor segunda forma fundamental da superfície $\Sigma$.

Além disto, sendo $N$ o campo vetorial normal unitário de tipo tempo apontando para o futuro $\left(x_{3}>0\right)$ em $\Sigma$, define-se em $\Sigma$ um campo tensorial $A: \mathcal{X}(\Sigma) \rightarrow \mathcal{X}(\Sigma)$ tal que

$$
\langle A X, Y\rangle=\langle\sigma(X, Y), N\rangle \forall X, Y \in \mathcal{X}(\Sigma) .
$$

Definição 1.3.5. A é chamado o operador forma, ou o operador de Weingarten, associado a $N$.

Note que o operador de Weingarten $A$ determina para cada ponto $p \in \Sigma$ um operador linear $A_{p}: T_{p} \Sigma \rightarrow T_{p} \Sigma$.

Adaptando as equações fundamentais para variedades semi-Riemannianas encontradas, por exemplo, em [23], escrevemos as equações fundamentais de $\Sigma$, conhecidas, 
respectivamente, como fórmula de Gauss, fórmula de Weingarten e equação de Codazzi.

$$
\begin{gathered}
\nabla_{X}^{\circ} Y=\nabla_{X} Y-\langle A X, Y\rangle N \\
A(X)=-\nabla_{X}^{\circ} N \\
\nabla A(X, Y)=\nabla A(Y, X)
\end{gathered}
$$

para quaisquer campos $X, Y \in \mathcal{X}(\Sigma)$

\section{Curvatura Média e Curvatura Gaussiana}

Definição 1.3.6. Definimos a função curvatura média como sendo

$$
H=-\frac{1}{2} \operatorname{traço(A)}
$$

onde $A$ é o operador forma associado a $N$. O vetor curvatura média é dado por

$$
\mathbf{H}=\frac{1}{2} \text { traço }(\sigma) \text {, onde } \sigma \text { é a segunda forma fundamental e, então, } \mathbf{H}=H N
$$

Definição 1.3.7. A função curvatura Gaussiana é dada por

$$
K=-\operatorname{det}(A) \text {. }
$$

A partir de agora, estudaremos principalmente as superfícies de tipo espaço que são compactas.

Apresentamos os conceitos e propriedades dessas superfícies, a serem fundamentados no decorrer da dissertação.

Lema 1.3.8. Toda superfície compacta de tipo espaço no $\mathbb{L}^{3}$ tem necessariamente bordo não vazio, ou equivalentemente, não existem superfícies de tipo espaço fechadas (compactas e sem bordo) no $\mathbb{L}^{3}$.

Prova: Suponha que $\Sigma$ é uma superfície compacta de tipo espaço e sem bordo, ou seja, fechada. Então os normais euclideanos unitários a $\Sigma$ cobrem toda a esfera $S^{2}(1)$, isto é, $\Sigma$ tem normais euclideanos em todas as direções. Seja $\tilde{N}$ o campo normal euclideano de $\Sigma$. Em coordenadas canônicas, $\tilde{N}$ pode ser escrito como $\tilde{N}=(a, b, c)$, $a, b, c \in \mathbb{R}$. Da mesma forma, o campo normal lorentziano de tipo tempo $N$ é escrito, 
em coordenadas, como $N=(a, b,-c)$. Portanto, $N$ também cobre a esfera $S^{2}(1)$. Em particular, se $c=0$, temos que $a^{2}+b^{2}=1$. Por outro lado, do fato de $N$ ser de tipo tempo, $a^{2}+b^{2}<0$, o que é um absurdo. Logo $\Sigma$ não pode ter normais euclideanos em todas as direções e portanto não pode ser fechada.

Dizemos que uma superfície compacta de tipo espaço imersa no $\mathbb{L}^{3}$ tem bordo se ela é a imagem pela imersão de tipo espaço $x$ de uma superfície compacta $\Sigma$ com bordo $\partial \Sigma$. Neste caso, o bordo $x(\partial \Sigma)$ é uma curva fechada $\Gamma$ e $x$ restrita a $\partial \Sigma$ é um difeomorfismo sobre $\Gamma$.

\section{Operadores Diferenciais}

Definimos o gradiente de uma função e o divergente de um campo em $\Sigma$, elementos que são bastante utilizados para a obtenção das fórmulas integrais encontradas no Capítulo 2.

Definição 1.3.9. O gradiente de uma função $f \in \mathcal{F}(\Sigma)$, denotado como $\nabla f$ é um campo vetorial metricamente equivalente à diferencial $d f \in \mathcal{X}^{*}(\Sigma)$. Ou seja,

$$
\langle\nabla f, X\rangle=d f(X)=X(f) \quad \forall X \in \mathcal{X}(\Sigma)
$$

Definição 1.3.10. Para cada $X \in \mathcal{X}(\Sigma)$, o divergente de $X$, denotado por div $(X)$ (ou Div $(X)$ ), sobre $\Sigma$ é dado por

$$
\operatorname{div}(X)=\operatorname{traço~}(\nabla X)
$$

onde $\nabla$ é a conexão Levi-Civita em $\Sigma$.

Teorema 1.3.11 (Teorema da Divergência). Sejam $\Sigma$ uma superfície compacta no $\mathbb{R}^{3}$ com bordo $\partial \Sigma$ e $\nu$ o co-normal unitário que aponta para dentro de $\Sigma$ ao longo de $\partial \Sigma$. Se G é um campo vetorial definido num conjunto aberto do $\mathbb{R}^{3}$ contendo $\Sigma$ então

$$
\int_{\Sigma} \operatorname{div}(G) d \Sigma=-\oint_{\partial \Sigma}\langle G, \nu\rangle d s
$$

onde d $\Sigma$ é o elemento de área de $\Sigma$ e ds é o elemento de comprimento em $\partial \Sigma$. 
Observamos que estas definições são as mesmas quando a dimensão da variedade é qualquer e mesmo o teorema da divergência pode ser reescrito para hipersuperfícies compactas. Além disto, este teorema pode ser usado no nosso contexto do $\mathbb{L}^{3}$ porque a métrica induzida em $\Sigma$ é Riemanniana.

\subsection{Alguns resultados de Análise}

Corolário 1.4.1 (Conseqüência da desigualdade de Hölder). Sejam $f, g$ funções contínuas no intervalo $[a, b]$ então

$$
\left(\int_{a}^{b} f\right)^{2} \leq(b-a) \int_{a}^{b}|f|^{2}
$$

Definição 1.4.2 (Aplicação de recobrimento). Sejam $\tilde{B}$ e $B$ subconjuntos de $\mathbb{R}^{3}$. Dizemos que $\pi: \tilde{B} \rightarrow B$ é uma aplicação de recobrimento se

1. $\pi$ é contínua e $\pi(\tilde{B})=B$

2. cada ponto $p \in B$ tem uma vizinhança $U$ em $B$ tal que

$$
\pi^{-1}(U)=\bigcup_{\alpha} V_{\alpha}
$$

onde os $V_{\alpha}$ são conjuntos abertos disjuntos dois a dois tais que a restrição de $\pi$ a $V_{\alpha}$ é um homeomorfismo de $V_{\alpha}$ sobre $U$.

Proposição 1.4.3. Seja $\pi: \tilde{B} \rightarrow B$ um homeomorfismo local, $\tilde{B}$ compacto e $B$ conexo. Então $\pi$ é uma aplicação de recobrimento.

Proposição 1.4.4. Seja $\pi: \tilde{B} \rightarrow B$ uma aplicação de recobrimento, $\tilde{B}$ conexo por arcos (caminhos) e B simplesmente conexo. Então $\pi$ é um homeomorfismo.

\subsection{Geometria Diferencial Complexa}

Nesta seção, pretendemos estudar alguns tópicos envolvendo variedades diferenciáveis complexas. Alguns desses resultados serão necessários no Capítulo 2, em que tomamos em $\Sigma$ uma estrutura quase complexa $J$. Baseamo-nos nas referências [14] e $[15]$. 


\section{Variedades Complexas}

Definição 1.5.1. Uma estrutura complexa numa variedade diferenciável $M$, de dimensão $2 n$, é uma coleção maximal de cartas diferenciáveis

$$
\left\{\left(U_{\lambda}, \varphi_{\lambda}\right): \lambda \in A, \varphi_{\lambda}: U_{\lambda} \rightarrow \mathbb{R}^{2 n}\right\} \text { tal que }
$$

1. $\bigcup_{\lambda \in A} U_{\lambda}=M$

2. $\varphi_{\lambda} \circ \varphi_{\mu}^{-1}: \varphi_{\mu}\left(U_{\lambda} \cap U_{\mu}\right) \rightarrow \varphi_{\lambda}\left(U_{\lambda} \cap U_{\mu}\right) \subset \mathbb{R}^{2 n}$ é uma função holomorfa considerada como uma função de um subconjunto aberto de $\mathbf{C}^{n}$ em $\mathbf{C}^{n}, \forall \lambda, \mu \in A$ com $U_{\lambda} \cap U_{\mu} \neq \emptyset$

Definição 1.5.2. Uma cobertura de uma variedade $M$ é uma coleção de conjuntos em $M$ cuja união é $M$.

Definição 1.5.3. Uma cobertura é aberta se todos os seus conjuntos são abertos.

Definição 1.5.4. Uma cobertura $\left\{V_{\beta}\right\}$ de uma variedade $M$ é dita um refinamento de uma cobertura $\left\{U_{\alpha}\right\}$ se para cada $V_{\beta}$ de $\left\{V_{\beta}\right\}$ existe um elemento $U_{\alpha}$ de $\left\{U_{\alpha}\right\}$ tal que $U_{\alpha}$ contém $V_{\beta}$.

Definição 1.5.5. Uma cobertura $\left\{U_{\alpha}\right\}$ de $M$ é dita localmente finita se para cada ponto $p \in M$ existe um aberto em $M$ contendo $p$ e intersectando somente um número finito de elementos de $\left\{U_{\alpha}\right\}$.

Definição 1.5.6. Uma variedade $M$ é paracompacta se é Hausdorff e se toda cobertura de $M$ tem um refinamento aberto localmente finito.

Definição 1.5.7 (Variedade Complexa). Uma variedade complexa é uma variedade paracompacta diferenciável junto com uma estrutura complexa.

Lema 1.5.8. Toda variedade compacta Hausdorff é paracompacta.

Teorema 1.5.9. Toda variedade Riemanniana de dimensão 2, orientada, conexa $e$ Hausdorff admite uma estrutura complexa. 
Prova: Considere o atlas $\mathcal{A}$ que contém todos os sistemas coordenados isotérmicos cujas mudanças de cartas têm determinante positivo. $\operatorname{Sejam}(U, \varphi)$ e $(V, \psi), \operatorname{com} \varphi=$ $(x, y)$ e $\psi=(u, v)$ dois sistemas de coordenadas de $\mathcal{A}$ cujos domínios se sobrepõem. Temos que mostrar que $\varphi \circ \psi^{-1}$ é holomorfa. Para isso, escrevemos

$$
\begin{aligned}
& \text { (1) } \frac{\partial}{\partial u}=\frac{\partial x}{\partial u} \frac{\partial}{\partial x}+\frac{\partial y}{\partial u} \frac{\partial}{\partial y} \\
& \text { (2) } \frac{\partial}{\partial v}=\frac{\partial x}{\partial v} \frac{\partial}{\partial x}+\frac{\partial y}{\partial v} \frac{\partial}{\partial y}
\end{aligned}
$$

De (1) e (2) e do fato da métrica ser conforme, temos que existe uma função $r(u, v)$ tal que

$$
\begin{aligned}
& \left(\frac{\partial x}{\partial u}\right)^{2}+\left(\frac{\partial y}{\partial u}\right)^{2}=r^{2} \\
& \left(\frac{\partial x}{\partial v}\right)^{2}+\left(\frac{\partial y}{\partial v}\right)^{2}=r^{2}
\end{aligned}
$$

e portanto existem duas funções diferenciáveis $\alpha, \beta$ com valores em $[0,2 \pi)$ tais que

$$
\begin{array}{ll}
\frac{\partial x}{\partial u}=r \cos \alpha & \frac{\partial y}{\partial u}=r \operatorname{sen} \alpha \\
\frac{\partial x}{\partial v}=r \cos \beta & \frac{\partial y}{\partial v}=r \operatorname{sen} \beta
\end{array}
$$

Usando que as coordenadas são isotérmicas, $\left\langle\frac{\partial}{\partial u}, \frac{\partial}{\partial v}\right\rangle=0$ obtemos $\cos (\alpha-\beta)=0 \Rightarrow$ $\alpha-\beta=\frac{\pi}{2}$ ou $\alpha-\beta=\frac{3 \pi}{2}$.

Mas, como a mudança de coordenadas tem determinante positivo, segue que

$$
\alpha-\beta=\frac{3 \pi}{2} .
$$

Além disto, $\cos \beta=-\operatorname{sen} \alpha, \operatorname{sen} \beta=\cos \alpha$.

Assim

$$
\begin{gathered}
\frac{\partial x}{\partial u}=r \sin \beta=\frac{\partial y}{\partial v} \\
\frac{\partial y}{\partial u}=-r \cos \beta=-\frac{\partial x}{\partial v}
\end{gathered}
$$

que são as equações de Cauchy-Riemann. Portanto $\varphi \circ \psi^{-1}$ é holomorfa. 


\section{Estrutura quase complexa}

Definição 1.5.10. Seja $M$ uma variedade diferenciável. Uma estrutura quase complexa em $M$ é um tensor diferenciável $J$ em $M$ tal que para cada $p \in M, J_{p}^{2}(v)=-v$ para todo $v \in T_{p} M$.

Observação 1.5.11. Um espaço vetorial real finito que admite um endomorfismo $J: V \rightarrow V$ satisfazendo $J^{2}=-I d$ tem necessariamente dimensão par.

Teorema 1.5.12. Toda variedade complexa de dimensão $2 n$ admite uma estrutura quase complexa.

Para a prova deste teorema, sugerimos [14, pág 241].

Pelo teorema 1.5.9, a superfície compacta de tipo espaço $\Sigma$ pode ser vista como uma variedade complexa de dimensão 2 , ou seja, é uma superfície de Riemann e pelo teorema 1.5.12, podemos tomar uma estrutura quase complexa em $\Sigma$.

O que vamos fazer agora é relacionar essa estrutura quase complexa com a métrica da superfície.

\section{Métricas Hermitiana e Kähleriana}

Definição 1.5.13. Uma métrica Riemanniana diferenciável g numa variedade complexa $M$ é dita Hermitiana se para cada $p \in M$ e cada par de vetores tangentes $u, v \in T_{p} M$

$$
g(u, v)=g(J u, J v)
$$

Definição 1.5.14. Se g é uma métrica Hermitiana, a 2-forma $w$ definida por $w(X, Y)=$ $g(J X, Y)$ é a forma de Kähler de $g$.

Definição 1.5.15. Uma métrica Hermitiana g numa variedade complexa é uma métrica Kähleriana se a forma de Kähler $w$ associada a g é fechada, isto é, dw $=0$.

Proposição 1.5.16. Seja $M$ uma variedade complexa e g uma métrica Hermitiana em $M$. Então as seguintes condições são equivalentes 
1. g é uma métrica Kähleriana

2. Se $\nabla$ é a derivada covariante associada a $g$, então $\nabla J=0$.

\section{Prova:}

$(1) \rightarrow(2)$ para todos $X, Y, Z$ vale que:

$$
\left\langle\left(\nabla_{Z} J\right) X, Y\right\rangle=\frac{1}{2} d w(X, Y, Z)-\frac{1}{2} d w(J X, J Y, Z) .
$$

Então, de $d w(X, Y, Z)=0 \forall X, Y, Z$, temos $\nabla J=0$.

$(2) \rightarrow(1)$ Vale para todos $X, Y, Z$

$$
3 d w(X, Y, Z)=\left\langle Y,\left(\nabla_{X} J\right)(Z)\right\rangle+\left\langle Z,\left(\nabla_{Y} J\right)(X)\right\rangle+\left\langle X,\left(\nabla_{Z} J\right)(Y)\right\rangle
$$

Então, de $\nabla J=0$, segue que $d w=0$.

\subsection{Algumas notas sobre curvas no $\mathbb{R}^{3}$}

Registramos aqui as notações da teoria de curvas que serão utilizadas no Capítulo 2, especialmente quando tratarmos do caso em que o bordo da superfície é uma curva plana.

Seja $\Gamma$ uma curva no $\mathbb{R}^{3}$. Uma parametrização de $\Gamma$ pelo comprimento de arco é uma aplicação diferenciável $\alpha: I \subset \mathbb{R} \rightarrow \mathbb{R}^{3}, I$ intervalo aberto de $\mathbb{R}$, tal que $\left\|\alpha^{\prime}(s)\right\|=1$.

Denotamos por $\tau(s)=\tau(\alpha(s))=\alpha^{\prime}(s), k(s)$ a curvatura de $\alpha(s)$ e $\eta(s)$ o campo normal unitário na direção de $\alpha^{\prime \prime}(s)$.

Uma conseqüência de $\alpha$ ser uma curva parametrizada pelo comprimento de arco é que $\eta$ é normal a $\alpha^{\prime}(s)$.

Podemos definir para uma curva parametrizada plana $\alpha: I \rightarrow \mathbb{R}^{3}$ a curvatura com sinal $k$. Para isso, seja $\left\{e_{1}, e_{2}\right\}$ a base natural do $\mathbb{R}^{2}$ e defina o normal $\eta(s), s \in I$ exigindo que a base $\{\tau(s), \eta(s)\}$ tenha a mesma orientação que a base $\left\{e_{1}, e_{2}\right\}$. A curvatura $k$ é então definida como $\tau^{\prime}(s)=k(s) \eta(s)$ e pode ser positiva ou negativa. (Figura 1.3) 
$e_{2}$
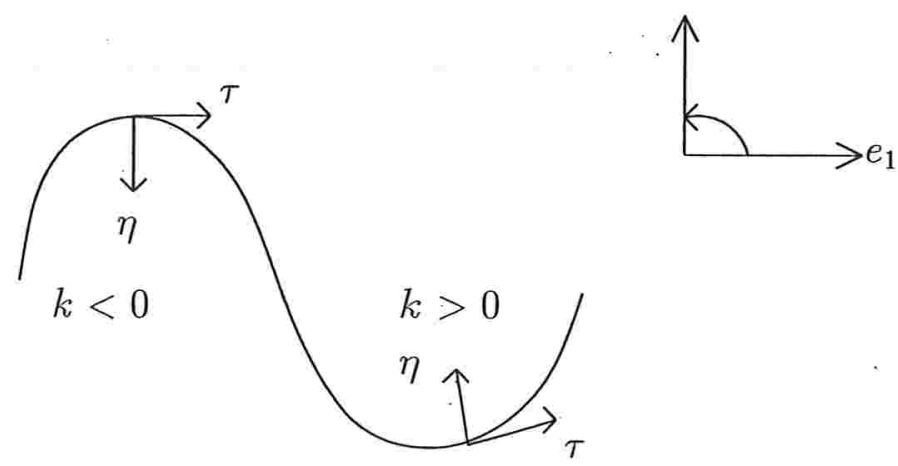

Figura 1.3: Curvatura com'sinal

Denotamos por $k_{n}(s)$ e $k_{g}(s)$ as curvaturas normal e geodésica, respectivamente de $\alpha(s)$, onde $\alpha$ é uma curva contida numa superfície.

Deste modo, no nosso contexto, sendo $\nu$ o co-normal unitário apontando para dentro de $\Sigma$ ao longo do bordo $\partial \Sigma$ e $N$ o normal unitário a $\Sigma$, como veremos no corolário 2.6.4, temos

$$
\alpha^{\prime \prime}(s)=k_{g}(s) \nu(s)+k_{n}(s) N(s)
$$




\section{Capítulo 2}

\section{Fórmulas Integrais}

Neste capítulo, desenvolvemos três fórmulas integrais que, combinadas, geram uma fórmula do fluxo e uma desigualdade integral para superfícies compactas de tipo espaço com curvatura média constante que são necessárias para obtermos os resultados pretendidos.

Seja $x: \Sigma \rightarrow \mathbb{L}^{3}$ uma superfície compacta de tipo espaço, orientada por um campo vetorial, normal e unitário, de tipo tempo $N$ apontando para o futuro.

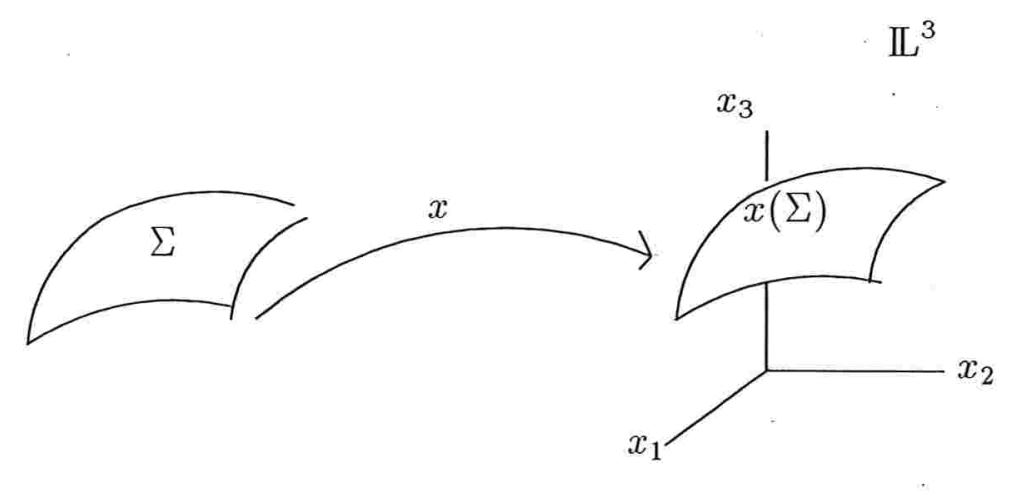

Figura 2.1: Representação da superfície imersa no $\mathbb{L}^{3}$

Como $\mathbb{L}^{3}$ é orientável existe uma 3 -forma $d V$ (chamada o elemento de volume) tal que se $\left\{e_{1}, e_{2}, e_{3}\right\}$ é uma base ortonormal do $\mathbb{L}^{3}$, então

$$
d V\left(e_{1}, e_{2}, e_{3}\right)= \pm 1
$$


Se o resultado é negativo, a base é dita orientada postivamente.

O mesmo vale para $\Sigma$. Considere $d \Sigma$ o elemento de área (que é o elemento de volume bidimensional) com respeito à métrica induzida e à orientação escolhida, isto é,

$$
d \Sigma_{p}(v, w)=d V\left(d x_{p}(v), d x_{p}(w), N(p)\right), v, w \in T_{p} \Sigma
$$

Tomemos $J: \mathcal{X}(\Sigma) \rightarrow \mathcal{X}(\Sigma)$ a estrutura quase complexa de $\Sigma$ e $w$ a forma de Kähler associada à métrica $\langle$,$\rangle . Então$

$$
w(X, Y)=\langle J X, Y\rangle
$$

para todos campos vetoriais tangentes $X, Y \in \mathcal{X}(\Sigma)$.

Por outro lado, $\langle J X, Y\rangle=d \Sigma(X, Y)$. Portanto $w$ é fechada, ou seja, a métrica $\langle$,$\rangle é Kähleriana.$

\subsection{A orientação induzida de $\partial \Sigma$}

Teorema 2.1.1. Seja $k>1$. Se $M$ é uma k-variedade orientável com bordo não vazio, então $\partial M$ é orientável.

O teorema acima assegura que o bordo de $\Sigma$ é orientável. Uma demonstração pode ser encontrada em [22].

A orientação de $\Sigma$ induz uma orientação natural em $\partial \Sigma$ que é descrita abaixo.

Um vetor não nulo tangente $v \in T_{p}(\partial \Sigma)$ está orientado positivamente se e somente se $\{v, w\}$ é uma base orientada positivamente para $T_{p} \Sigma$ sempre que $w \in T_{p} \Sigma$ está apontando para dentro de $\Sigma$.

Denotamos por $\nu$ o vetor co-normal unitário apontando para dentro de $\Sigma$ ao longo de $\partial \Sigma$, enquanto $\tau$ é o campo vetorial unitário tangente orientado positivamente ao longo de $\partial \Sigma$, que é dado por $\tau=-J(\nu)$. 


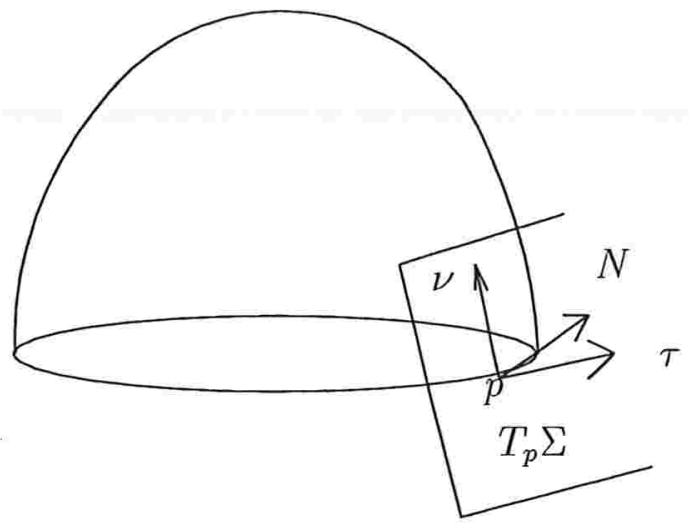

Figura 2.2: Orientação de $\partial \Sigma$

\subsection{A primeira fórmula integral}

Seja $a \in \mathbb{L}^{3}$ um vetor fixado arbitrariamente decomposto, ao longo da imersão, como

$$
a=a^{T}+a^{\perp}
$$

onde $a^{T} \in \mathcal{X}(\Sigma)$ é tangente a $\Sigma$ e $a^{\perp}$ é a parte de $a$ na direção do normal $N$.

Então

$$
a^{\perp}=\operatorname{proj}_{N} a=\frac{\langle a, N\rangle}{\langle N, N\rangle} N=-\langle a, N\rangle N .
$$

Reescrevemos,

$$
a=a^{T}-\langle a, N\rangle N .
$$

Vamos calcular $\operatorname{div}\left(a^{T}\right)$. Para isso, precisamos obter $\nabla_{X} a^{T}$.

Usando que $\nabla^{\circ} a=0$, temos:

$$
\begin{gathered}
0=\left(\nabla_{X}^{\circ} a\right)^{T}=\left(\nabla_{X}^{\circ} a^{T}\right)^{T}-\left(\nabla_{X}^{\circ}\langle a, N\rangle N\right)^{T}=\nabla_{X} a^{T}-\langle a, N\rangle \nabla_{X}^{\circ} N= \\
=\nabla_{X} a^{T}+\langle a, N\rangle A(X) \\
\therefore \nabla_{X} a^{T}=-\langle a, N\rangle A(X)
\end{gathered}
$$

$\forall X \in \mathcal{X}(\Sigma)$.

Logo $\operatorname{div}\left(a^{T}\right)=\operatorname{traço}\left(\nabla a^{T}\right)=\operatorname{traço}(-\langle a, N\rangle A)=-\langle a, N\rangle \operatorname{traço}(A)=2 H\langle a, N\rangle$.

Aplicando o teorema da divergência, obtemos a primeira fórmula integral: 
Proposição 2.2.1 (A $1^{a}$ fórmula integral). Seja $x: \Sigma \rightarrow \mathbb{L}^{3}$ uma imersão de tipo espaço de uma superfície compacta com bordo $\partial \Sigma$ orientada por um campo normal unitário $N$ de tipo tempo apontando para o futuro. Se $H$ é a função curvatura média de $\Sigma$, então para qualquer vetor fixado arbitrariamente $a \in \mathbb{L}^{3}$,

$$
2 \int_{\Sigma} H\langle a, N\rangle d \Sigma=-\oint_{\partial \Sigma}\langle a, \nu\rangle d s
$$

onde $\nu$ é o vetor co-normal unitário que aponta para dentro de $\Sigma$ ao longo de $\partial \Sigma$.

\section{Prova:}

$$
\int_{\Sigma} 2 H\langle a, N\rangle d \Sigma=\int_{\Sigma} \operatorname{div}\left(a^{T}\right) d \Sigma=-\oint_{\partial \Sigma}\left\langle a^{T}, \nu\right\rangle d s=-\oint_{\partial \Sigma}\langle a, \nu\rangle d s
$$

\subsection{A segunda fórmula integral}

Vamos calcular $\operatorname{div}\left(A\left(a^{T}\right)+H a^{T}\right)$. Para isso, sendo $A$ um tensor de tipo (1,1) (pela observação 1.2.8), podemos considerar a sua derivada covariante de primeira ordem dada por:

$$
(\nabla A)(X, Y)=\left(\nabla_{Y} A\right)(X)=\nabla_{Y}(A X)-A\left(\nabla_{Y} X\right)
$$

Usando a equação de Codazzi (1.3) e as equações (2.2) e (2.4), segue

$$
\begin{aligned}
\operatorname{div}\left(A\left(a^{T}\right)\right) & =\operatorname{traço}\left\{X \mapsto \nabla_{X}\left(A\left(a^{T}\right)\right\}=\right. \\
& =\operatorname{traço}\left\{X \mapsto\left(\nabla_{X} A\right) a^{T}\right\}+\operatorname{traço}\left\{X \mapsto A\left(\nabla_{X} a^{T}\right)\right\}= \\
& =\operatorname{traço}\left\{X \mapsto\left(\nabla_{a^{T}} A\right) X\right\}+\operatorname{traço}\{X \mapsto A(-\langle N, a\rangle A X)\}= \\
& =\operatorname{traço}\left(\nabla_{a^{T}} A\right)-\langle N, a\rangle \operatorname{traço}\left(A^{2}\right) .
\end{aligned}
$$

Calculemos separadamente os valores dessas duas parcelas:

$$
\begin{aligned}
\operatorname{traço}\left(\nabla_{a^{T}} A\right) & =\operatorname{traço}\left\{X \mapsto \nabla_{a^{T}}(A X)\right\}-\operatorname{traço}\left\{X \mapsto A\left(\nabla_{a^{T}} X\right)\right\}= \\
& =\sum_{i=1}^{2}\left[\left\langle\nabla_{a^{T}}\left(A\left(E_{i}\right)\right), E_{i}\right\rangle-\left\langle A\left(\nabla_{a^{T}} E_{i}\right), E_{i}\right\rangle\right]=
\end{aligned}
$$




$$
=\sum_{i=1}^{2}\left[\left\langle\nabla_{a^{T}}\left(A\left(E_{i}\right)\right), E_{i}\right\rangle-\left\langle\nabla_{a^{T}} E_{i}, A\left(E_{i}\right)\right\rangle\right]
$$

onde $\left\{E_{1}, E_{2}\right\}$ é um referencial local ortonormal de campos em $\Sigma$.

Seguindo o que foi feito em [11], para cada $p_{0} \in \Sigma$ é possível escolhermos um referencial local ortonormal $\left\{e_{1}, e_{2}\right\}$ verificando $\left(\nabla_{e_{i}} e_{j}\right)\left(p_{0}\right)=0, \forall i, j=1,2$. Basta tomarmos uma base associada ao sistema de coordenadas normal dado em uma vizinhança normal de cada $p_{0}$ e ortonormalizá-lo.

Tomando então um referencial ortonormal com essas características:

$\operatorname{traço}\left(\nabla_{a^{T}} A\right)\left(p_{0}\right)=\sum\left\langle\nabla_{a^{T}}\left(A\left(e_{i}\right)\right), e_{i}\right\rangle\left(p_{0}\right)$ e $a^{T}(\operatorname{traço} A)\left(p_{0}\right)=\sum\left\langle\nabla_{a^{T}}\left(A\left(e_{i}\right)\right), e_{i}\right\rangle\left(p_{0}\right)$.

Como isso vale para qualquer ponto $p_{0} \in \Sigma$ segue que:

$\operatorname{traço}\left(\nabla_{a^{T}} A\right)=a^{T}(\operatorname{traço}(A))=a^{T}(-2 H)=-2\left\langle\nabla H, a^{T}\right\rangle=-2\langle\nabla H, a\rangle$, onde $\nabla H$ é o gradiente da função $H$.

Para encontrarmos o $\operatorname{traço}\left(A^{2}\right)$, utilizaremos a equação de Cayley-Hamilton para o operador forma $A$ dada por

$$
A^{2}-\operatorname{traço}(A) A+\operatorname{det}(A) I_{2}=0,
$$

ou seja,

$$
A^{2}+2 H A-K I_{2}=0
$$

onde $H$ e $K$ são, respectivamente, as curvaturas média e Gaussiana de $\Sigma$.

$$
\begin{aligned}
& \text { Assim, } \operatorname{traço~}\left(A^{2}\right)=4 H^{2}+2 K \\
& \text { Logo, } \operatorname{div}\left(A\left(a^{T}\right)\right)=-2\langle\nabla H, a\rangle-\left(4 H^{2}+2 K\right)\langle N, a\rangle . \\
& \begin{aligned}
\operatorname{div}\left(H a^{T}\right) & =\operatorname{traço}\left\{X \mapsto \nabla_{X}\left(H a^{T}\right)\right\}= \\
& =\operatorname{traço}\left\{X \mapsto \nabla_{X} H a^{T}\right\}+H \operatorname{traço}\left\{X \mapsto \nabla_{X} a^{T}\right\}= \\
& =\left\langle\left(\nabla_{E_{1}} H\right) a^{T}, E_{1}\right\rangle+\left\langle\left(\nabla_{E_{2}} H\right) a^{T}, E_{2}\right\rangle+2 H^{2}\langle a, N\rangle= \\
& =E_{1}(H) a_{1}^{T}+E_{2}(H) a_{2}^{T}+2 H^{2}\langle a, N\rangle= \\
& =a^{T}(H)+2 H^{2}\langle a, N\rangle=\left\langle\nabla H, a^{T}\right\rangle+2 H^{2}\langle a, N\rangle= \\
& =\langle\nabla H, a\rangle+2 H^{2}\langle a, N\rangle
\end{aligned}
\end{aligned}
$$


onde $\left\{E_{1}, E_{2}\right\}$ é um referencial local ortonormal e $a^{T}=a_{1}^{T} E_{1}+a_{2}^{T} E_{2}$.

$$
\therefore \operatorname{div}\left(A\left(a^{T}\right)+H a^{T}\right)=-\langle\nabla H, a\rangle-\left(2 H^{2}+2 K\right)\langle N, a\rangle .
$$

Aplicando novamente o teorema da divergência, chegamos a:

Proposição 2.3.1 (A $2^{a}$ fórmula integral). Seja $x: \Sigma \rightarrow \mathbb{L}^{3}$ uma imersão de tipo espaço de uma superfície compacta com bordo $\partial \Sigma$ orientada por um campo normal unitário $N$ de tipo tempo apontando para o futuro. Se $H$ é a função curvatura média e $K$ é a curvatura Gaussiana de $\Sigma$, então para qualquer vetor fixado arbitrariamente $a \in \mathbb{L}^{3}$,

$$
\int_{\Sigma}\left\{\langle\nabla H, a\rangle+\left(2 H^{2}+2 K\right)\langle N, a\rangle\right\} d \Sigma=\oint_{\partial \Sigma}\left\langle A\left(a^{T}\right)+H a^{T}, \nu\right\rangle d s
$$

onde $\nu$ é o vetor co-normal unitário apontando para dentro de $\Sigma$ ao longo de $\partial \Sigma$.

\section{Prova:}

$-\int_{\Sigma} \operatorname{div}\left(A\left(a^{T}\right)+H a^{T}\right) d \Sigma=\int_{\Sigma}\left\{\langle\nabla H, a\rangle+\left(2 H^{2}+2 K\right)\langle N, a\rangle\right\} d \Sigma=\oint_{\partial \Sigma}\left\langle A\left(a^{T}\right)+H a^{T}, \nu\right\rangle d s$,

\subsection{A terceira fórmula integral}

Para um vetor fixado arbitrariamente $a \in \mathbb{L}^{3}$, consideremos agora o produto vetorial $x \wedge a \in \mathbb{L}^{3}$, onde $x$ é a imersão $x: \Sigma \rightarrow \mathbb{L}^{3}$. Escrevemos

$$
x \wedge a=(x \wedge a)^{T}-\langle x \wedge a, N\rangle N
$$

onde $(x \wedge a)^{T} \in \mathcal{X}(\Sigma)$ é tangente a $\Sigma$.

Nosso objetivo é calcular $\operatorname{div}\left(J(x \wedge a)^{T}\right)$ onde $J$ é a estrutura quase complexa em $\Sigma$.

$$
\operatorname{div}\left(J(x \wedge a)^{T}\right)=\operatorname{traço}\left(\nabla\left(J(x \wedge a)^{T}\right)\right)=\operatorname{traço}\left\{X \mapsto \nabla_{X} J(x \wedge a)^{T}\right\}
$$

Mas $J$ é um tensor $(1,1)$, então vale que: 


$$
\nabla\left(J(x \wedge a)^{T}\right)=(\nabla J)(x \wedge a)^{T}+J\left(\nabla(x \wedge a)^{T}\right)
$$

Além disto, já vimos que $w(X, Y)=\langle J X, Y\rangle$ é uma forma fechada, pois $w(X, Y)=$ $d \Sigma(X, Y)$ e $d w=d(d \Sigma)=0$. Logo, pela proposição 1.5 .16$, temos $\nabla J=0$.

Basta então calcularmos $\nabla_{X}(x \wedge a)^{T}$ para $X \in \mathcal{X}(\Sigma)$.

Usando as fórmulas de Gauss e Weingarten e a equação (2.7):

$$
\begin{aligned}
\nabla_{X}(x \wedge a)^{T}= & \nabla_{X}^{\circ}(x \wedge a)^{T}+\left\langle A X,(x \wedge a)^{T}\right\rangle N= \\
= & \nabla_{X}^{\circ}(x \wedge a)+\nabla_{X}^{\circ}(\langle x \wedge a, N\rangle N)+\langle A X, x \wedge a\rangle N= \\
= & \left(\nabla_{X}^{\circ} x\right) \wedge a+\left\langle\left(\nabla_{X}^{\circ} x\right) \wedge a, N\right\rangle N+\left\langle x \wedge a, \nabla_{X}^{\circ} N\right\rangle N+ \\
& \quad+\langle x \wedge a, N\rangle \nabla_{X}^{\circ} N+\langle A X, x \wedge a\rangle N= \\
= & X \wedge a+\langle X \wedge a, N\rangle N-\langle x \wedge a, A X\rangle N+ \\
& +\langle x \wedge a, N\rangle(-A X)+\langle A X, x \wedge a\rangle N= \\
= & (X \wedge a)^{T}-\langle x \wedge a, N\rangle A X .
\end{aligned}
$$

Deste modo

$$
\begin{aligned}
\operatorname{div}\left(J(x \wedge a)^{T}\right)= & \operatorname{traço}\left\{X \mapsto J\left((X \wedge a)^{T}-\langle x \wedge a, N\rangle A X\right)\right\}= \\
= & \left\langle J\left(E_{1} \wedge a\right)^{T}, E_{1}\right\rangle+\left\langle J\left(E_{2} \wedge a\right)^{T}, E_{2}\right\rangle+ \\
& -\langle x \wedge a, N\rangle\left\{\left\langle J\left(A\left(E_{1}\right)\right), E_{1}\right\rangle+\left\langle J\left(A\left(E_{2}\right)\right), E_{2}\right\rangle\right\}= \\
= & -\left\langle\left(E_{1} \wedge a\right)^{T}, J\left(E_{1}\right)\right\rangle-\left\langle\left(E_{2} \wedge a\right)^{T}, J\left(E_{2}\right)\right\rangle+ \\
& +\langle x \wedge a, N\rangle\left\{\left\langle A\left(E_{1}\right), J\left(E_{1}\right)\right\rangle+\left\langle A\left(E_{2}\right), J\left(E_{2}\right)\right\rangle\right\}= \\
= & -\left\langle E_{1} \wedge a, E_{2}\right\rangle+\left\langle E_{2} \wedge a, E_{1}\right\rangle+ \\
& +\langle x \wedge a, N\rangle\left\{\left\langle A\left(E_{1}\right), E_{2}\right\rangle-\left\langle A\left(E_{2}\right), E_{1}\right\rangle\right\}= \\
= & \left\langle E_{1} \wedge E_{2}, a\right\rangle+\left\langle E_{1} \wedge E_{2}, a\right\rangle= \\
= & 2\left\langle E_{1} \wedge E_{2}, a\right\rangle=-2\langle N, a\rangle
\end{aligned}
$$

Integrando em $\Sigma$ e usando novamente o teorema da divergência e o fato de que $J$ é anti auto-adjunto, encontramos 
Proposição 2.4.1 (A $3^{a}$ fórmula integral). Seja $x: \Sigma \rightarrow \mathbb{L}^{3}$ uma imersão de tipo espaço de uma superfície compacta com bordo $\partial \Sigma$ orientada por um campo normal unitário $N$ de tipo tempo apontando para o futuro. Se $H$ é a função curvatura média de $\Sigma$, então para qualquer vetor fixado arbitrariamente $a \in \mathbb{L}^{3}$,

$$
2 \int_{\Sigma}\langle N, a\rangle d \Sigma=-\oint_{\partial \Sigma}\langle x \wedge \tau, a\rangle d s
$$

onde $\tau$ é o vetor unitário tangente orientado positivamente ao longo de $\partial \Sigma$.

Prova:

$$
\begin{aligned}
-\int_{\Sigma} \operatorname{div}\left(J(x \wedge a)^{T}\right) d \Sigma & =2 \int_{\Sigma}\langle N, a\rangle d \Sigma=\oint_{\partial \Sigma}\left\langle J(x \wedge a)^{T}, \nu\right\rangle d s= \\
& =\oint_{\partial \Sigma}\left\langle(x \wedge a)^{T},-J(\nu)\right\rangle d s=\oint_{\partial \Sigma}\left\langle(x \wedge a)^{T}, \tau\right\rangle d s= \\
& =\oint_{\partial \Sigma}\langle x \wedge a, \tau\rangle d s=-\oint_{\partial \Sigma}\langle x \wedge \tau, a\rangle d s .
\end{aligned}
$$

\subsection{Fórmula do fluxo}

A fórmula do fluxo para superfícies de tipo espaço com curvatura média constante $H$ imersas no $\mathbb{L}^{3}$ vem como uma primeira aplicação dessas fórmulas integrais.

Lema 2.5.1 (Fórmula do fluxo). Seja $x: \Sigma \rightarrow \mathbb{L}^{3}$ uma imersão de tipo espaço de uma superfície compacta com bordo $\partial \Sigma$. Se a curvatura média $H$ é constante, então para qualquer vetor fixado a $\in \mathbb{L}^{3}$ temos

$$
\oint_{\partial \Sigma}\langle a, \nu\rangle d s=H \oint_{\partial \Sigma}\langle x \wedge \tau, a\rangle d s
$$

onde $\tau$ é o vetor unitário tangente orientado positivamente ao longo de $\partial \Sigma$ e $\nu$ é o vetor co-normal e unitário que aponta para dentro de $\Sigma$ ao longo de $\partial \Sigma$.

Prova: Da primeira e da terceira fórmulas integrais

$$
\oint_{\partial \Sigma}\langle a, \nu\rangle d s=-2 H \int_{\Sigma}\langle a, N\rangle d \Sigma=H \oint_{\partial \Sigma}\langle x \wedge \tau, a\rangle d s
$$




\subsection{Conseqüências da fórmula do fluxo para bordo planar}

Observamos que se $\Gamma=x(\partial \Sigma)$ então $\Gamma$ é uma curva fechada pois a restrição de $x$ ao bordo $\partial \Sigma$ é um difeomorfismo sobre $\Gamma \subset \mathbb{L}^{3}$.

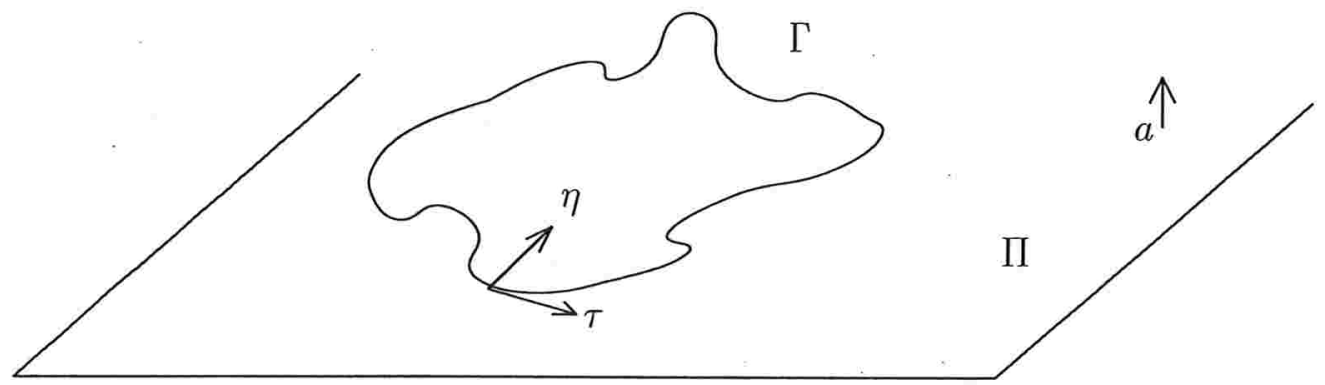

Figura 2.3: Representação de $\partial \Sigma$ planar

Além disso, se $\Pi$ é o plano contendo $\Gamma$, então $\Pi$ é de tipo espaço. Basta notar que o fato de $\Gamma$ ser fechada implica que o campo normal $\eta$ da curva cobre um círculo. Suponha que em algum ponto $p \in \Gamma$, o vetor $\eta(p)$ seja de tipo tempo, isto é, pertença à interseção do círculo com o interior do cone de luz. À medida em que $\eta$ percorre o círculo, o campo tangente $\tau$ atinge, para algum ponto do bordo de $\Sigma$, o interior do cone de luz, contradizendo o fato de que $\tau$ pertence a $T_{p} \Sigma$ para todo $p \in \Sigma$ ( $\tau$ é de tipo espaço). Logo o campo normal $\eta$ é sempre de tipo espaço e, das propriedades do produto vetorial descritas no Capítulo $1, \tau \wedge \eta$ é de tipo tempo, que é o normal ao plano. Conseqüentemente, $\Pi$ é de tipo espaço.

Podemos assumir que $\Pi$ passa pela origem e $\Pi=a^{\perp}$ onde $a \in \mathbb{L}^{3}$ é um vetor unitário de tipo tempo apontando para o futuro.

Então $\langle a, a\rangle=-1$ e como $a$ pertence ao cone de tipo tempo contendo $N$. vale que $|\langle N, a\rangle| \geq|N||a|=1 \Rightarrow\langle N, a\rangle \leq-1<0$, do lema 1.1.3 e da proposição 1.1.4.

Consideremos a orientação natural do plano $\Pi$ determinada por $a$. Segue da terceira fórmula integral (proposição 2.4.1) que

$$
\oint_{\partial \Sigma}\langle x \wedge \tau, a\rangle d s=-2 \int_{\Sigma}\langle N, a\rangle d \Sigma=2 \int_{\Sigma}-\langle N, a\rangle d \Sigma \geq 2 \int_{\Sigma} d \Sigma=2 \text { área }(\Sigma)>0
$$


O próximo resultado diz que o fluxo não depende da superfície $\Sigma$ mas somente do valor da curvatura média $H$ e de $\Gamma$.

Corolário 2.6.1. Seja $x: \Sigma \rightarrow \mathbb{L}^{3}$ uma imersão de tipo espaço de uma superfície compacta limitada por uma curva de Jordan plana $\Gamma$. Seja a o vetor unitário de tipo tempo apontando para o futuro no $\mathbb{L}^{3}$ tal que $\Gamma$ está contida no plano de tipo espaço $a^{\perp}$. Se a curvatura média $H$ de $\Sigma$ é constante, então

$$
\oint_{\partial \Sigma}\langle\nu, a\rangle d s=2 H \text { área }(\Omega)
$$

Prova: Se $\Gamma$ é uma curva de Jordan plana e $\Omega$ é o domínio no plano $\Pi$ limitado por $\Gamma$ então

$$
\operatorname{área}(\Omega)=-\frac{1}{2} \oint_{\partial \Sigma}\langle x, \eta\rangle d s
$$

Logo:

$$
\oint_{\partial \Sigma}\langle x \wedge \tau, a\rangle d s=\oint_{\partial \Sigma}\langle x, \tau \wedge a\rangle d s=-\oint_{\partial \Sigma}\langle x, \eta\rangle d s=2 \text { área }(\Omega)
$$

onde $\eta=-\tau \wedge a$ é o vetor unitário co-normal a $\Pi$ que aponta para dentro ao longo de $\Gamma$, isto é, $\eta$ é o vetor aceleração da curva.

Da fórmula do fluxo (lema 2.5.1)

$$
\oint_{\partial \Sigma}\langle\nu, a\rangle d s=H \oint_{\partial \Sigma}\langle x \wedge \tau, a\rangle d s=2 \operatorname{Hárea}(\Omega)
$$

Observação 2.6.2. O Corolário anterior não implica qualquer restrição aos valores possíveis da curvatura média constante. Por exemplo, a família de calotas hiperbólicas $\Sigma_{\rho}=\left\{\left(x_{1}, x_{2}, x_{3}\right) \in \mathbb{L}^{3} / x_{1}^{2}+x_{2}^{2}-x_{3}^{2}=-\rho^{2}, 0<x_{3} \leq \sqrt{r^{2}+\rho^{2}}\right\}$ onde $0<\rho<\infty$, descreve uma família de superfícies de tipo espaço compactas no $\mathrm{L}^{3}$, com curvatura média constante $H_{\rho}=\frac{1}{\rho}$, limitadas por um círculo de raio $r$, de modo que $0<H_{\rho}<$ $\infty$.

No entanto, no caso Euclideano, se $\Gamma$ é um círculo de raio $r>0$ e $\Sigma$ é uma superficie compacta imersa no $E^{3}$ limitada por $\Gamma$ com curvatura média constante $H$, então a fórmula correspondente do fluxo implica que $0 \leq|H| \leq \frac{1}{r}$ (ver [7], Corolário 2.2). 


\subsubsection{Uma desigualdade integral}

A segunda fórmula integral (2.6) para $H$ constante torna-se:

$$
\begin{aligned}
2 \int_{\Sigma}\left(H^{2}+K\right)\langle N, a\rangle d \Sigma & =\oint_{\partial \Sigma}\left\langle A\left(a^{T}\right)+H a^{T}, \nu\right\rangle d s= \\
& =\oint_{\partial \Sigma}\left\{\left\langle a^{T}, A(\nu)\right\rangle+H\left\langle a^{T}, \nu\right\rangle\right\} d s= \\
& =\oint_{\partial \Sigma}\{\langle a, A(\nu)\rangle+H\langle a, \nu\rangle\} d s
\end{aligned}
$$

Além disto, como $\Gamma=x(\partial \Sigma)$ é uma curva de Jordan plana contida em $\Pi=a^{\perp}$ onde $a \in \mathbb{L}^{3}$ é um vetor unitário de tipo tempo que aponta para o futuro e $\tau$ é o vetor tangente unitário orientado positivamente ao longo de $\Gamma$, então $\langle\tau, a\rangle=0$. Escrevendo a na base $\{\tau, \nu, N\}$ do $\mathbb{L}^{3}$ temos, ao longo de $\Gamma$,

$$
a=\langle a, \nu\rangle \nu-\langle a, N\rangle N
$$

Segue que

$$
\langle A(\nu), a\rangle=\langle A(\nu),\langle a, \nu\rangle \nu\rangle=\langle a, \nu\rangle\langle A(\nu), \nu\rangle
$$

Por outro lado, $\{\tau, \nu\}$ é um referencial ortonormal para $T_{p} \Sigma$, então

$$
-2 H=\operatorname{traço}(A)=\langle A(\nu), \nu\rangle+\langle A(\tau), \tau\rangle
$$

Mas $\langle A(\tau), \tau\rangle=-k_{n}$ onde $k_{n}$ é a curvatura normal ao longo de $\Gamma$.

Portanto $\langle A(\nu), a\rangle=\left(-2 H+k_{n}\right)\langle a, \nu\rangle$.

A segunda fórmula integral finalmente torna-se:

$2 \int_{\Sigma}\left(H^{2}+K\right)\langle N, a\rangle d \Sigma=\oint_{\partial \Sigma}\left(-2 H+k_{n}+H\right)\langle a, \nu\rangle d s=\oint_{\partial \Sigma}\left(k_{n}-H\right)\langle\nu, a\rangle d s$.

Observamos que $H^{2}+K=\frac{1}{2}\left\{\operatorname{traço}\left(A^{2}\right)-\frac{1}{2}(\operatorname{traço}(A))^{2}\right\}$, pois $\operatorname{traço}(A)=-2 H$ e $\operatorname{traço}\left(A^{2}\right)=4 H^{2}+2 K$. 
Lema 2.6.3. Sejam $H$ e $K$ as curvaturas média e Gaussiana, respectivamente, de $\Sigma$. Então $H^{2}+K \geq 0$ e a igualdade vale em um ponto $p \in \Sigma$ se e somente se $p$ é um ponto umbílico.

Prova: Seja $A: \mathcal{X}(\Sigma) \rightarrow \mathcal{X}(\Sigma)$ o endomorfismo de Weingarten e defina

$$
A^{\prime}=A-\frac{1}{2} \operatorname{traço}(A) I_{2}
$$

Como $A$ é diagonalizável então $A^{\prime}$ também o é e, portanto, $\left(A^{\prime}\right)^{2}$ é diagonalizável com todos os seus autovalores positivos, conseqüentemente, traço $\left(A^{\prime}\right)^{2} \geq 0$.

$\operatorname{Temos}\left(A^{\prime}\right)^{2}=\left(A-\frac{1}{2} \operatorname{traço}(A) I_{2}\right)^{2}=A^{2}-\operatorname{traço}(A) A+\frac{1}{4}(\operatorname{traço}(A))^{2} I_{2}$

Logo

$$
\begin{aligned}
0 \leq \operatorname{traço}\left(A^{\prime}\right)^{2} & =\operatorname{traço}\left(A^{2}\right)-(\operatorname{traço}(A))^{2}+\frac{1}{2}(\operatorname{traço}(A))^{2}= \\
& =\operatorname{traço}\left(A^{2}\right)-\frac{1}{2}(\operatorname{traço}(A))^{2}= \\
& =2\left(H^{2}+K\right)
\end{aligned}
$$

Se $p \in \Sigma$ é um ponto tal que $\left(H^{2}+K\right)(p)=0$, temos:

$\operatorname{traço}\left(A_{p}^{\prime}\right)^{2}=0 \Leftrightarrow A_{p}^{\prime}=0 \Leftrightarrow A_{p}=\lambda I_{2}$ onde $\lambda=\frac{1}{2} \operatorname{traço}\left(A_{p}\right)$. Isto significa que os autovalores de $A$ em $p$ são iguais e, portanto, as curvaturas principais $k_{1}(p)$ e $k_{2}(p)$ são idênticas, ou seja, $p$ é um ponto umbílico.

Como conseqüência do Lema acima e do fato de $\langle N, a\rangle \leq-1$ temos

Corolário 2.6.4. Seja $x: \Sigma \rightarrow \mathbb{L}^{3}$ uma imersão de tipo espaço de uma superfície compacta limitada por uma curva de Jordan plana $\Gamma$. Seja a o vetor unitário de tipo tempo apontando para o futuro no $\mathbb{L}^{3}$ tal que $\Gamma$ está contida no plano de tipo espaço $a^{\perp}$. Seja $\Omega$ o domínio planar limitado por $\Gamma$. Se a curvatura média $H$ é constante, então

$$
\oint_{\partial \Sigma} k_{n}\langle\nu, a\rangle d s \leq 2 H^{2} \text { área }(\Omega)
$$

onde $k_{n}$ é a curvatura normal ao longo do bordo. Além disto, a igualdade vale se e somente se a superfície é totalmente umbilica. 
Prova: Temos $\int_{\Sigma}\left(H^{2}+K\right)\langle N, a\rangle d \Sigma \leq 0$, de $\langle N, a\rangle \leq-1$ e do lema 2.6.3. Portanto da equação (2.10) e do Corolário 2.6.1

$$
\oint_{\partial \Sigma} k_{n}\langle\nu, a\rangle d s=H \oint_{\partial \Sigma}\langle\nu, a\rangle d s+2 \int_{\Sigma}\left(H^{2}+K\right)\langle N, a\rangle d \Sigma \leq 2 H^{2} a ́ r e a(\Omega) .
$$

A igualdade vale se e somente se $H^{2}+K=0$, ou seja, a igualdade vale se e só se $\Sigma$ é totalmente umbílica.

Este Corolário pode ser reescrito em termos da curvatura de $\Gamma$.

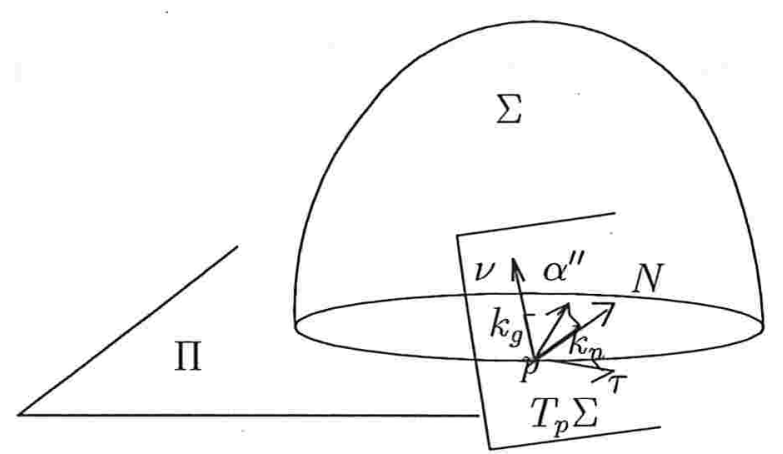

Figura 2.4: Representação das curvaturas normal e geodésica

Seja $\alpha$ uma parametrização pelo comprimento de arco (orientada positivamente) de $\Gamma$, de modo que $\alpha^{\prime}(s)=\tau(\alpha(s))=\tau(s)$. Seja $k$ a curvatura com sinal de $\alpha$ como uma curva plana. Então $\alpha^{\prime \prime}(s)=k(s) \eta(s)$, onde $\eta=-\tau \wedge a$ e $\eta(s)=\eta(\alpha(s))$. Além disso,

$$
\alpha^{\prime \prime}(s)=k_{g}(s) \nu(s)+k_{n}(s) N(s)
$$

onde $k_{g}$ é a curvatura geodésica de $\Gamma, \nu(s)=\nu(\alpha(s))$ e $N(s)=N(\alpha(s))$ pois $\left\langle\alpha^{\prime \prime}(s), \tau(s)\right\rangle=\left\langle\alpha^{\prime \prime}(s), \alpha^{\prime}(s)\right\rangle=0$.

$$
\begin{gathered}
\text { Como }\langle\eta, N\rangle=\langle-\tau \wedge a, N\rangle=\langle a, \tau \wedge N\rangle=-\langle\nu, a\rangle \text {, temos } \\
-k_{n}=\left\langle\alpha^{\prime \prime}, N\right\rangle=\langle k \eta, N\rangle=-k\langle\nu, a\rangle \\
\therefore k_{n}=k\langle\nu, a\rangle
\end{gathered}
$$

Desta forma, fica demonstrado um resultado análogo ao Corolário 2.6.4 
Corolário 2.6.5. Seja $x: \Sigma \rightarrow \mathbb{L}^{3}$ uma imersão de tipo espaço de uma superfície compacta limitada por uma curva de Jordan plana $\Gamma$. Seja a o vetor unitário de tipo tempo apontando para o futuro no $\mathbb{L}^{3}$ tal que $\Gamma$ está contida no plano de tipo espaço $a^{\perp}$. Seja $\Omega$ o dominio planar limitado por $\Gamma$. Se a curvatura média $H$ é constante, então

$$
\oint_{\partial \Sigma} k\langle\nu, a\rangle^{2} d s \leq 2 H^{2} \text { área }(\Omega) .
$$

onde $k$ é a curvatura com sinal de $\Gamma$. E a igualdade vale se e somente se a superfície é totalmente umbílica.

\subsection{O primeiro resultado de unicidade}

A proposição abaixo é importante para a prova do primeiro resultado de unicidade. Sua formulação é análoga à dada por Carmo em [10].

Proposição 2.7.1. Se todos os pontos de uma superfície de tipo espaço $\Sigma$ (conexa) imersa no $\mathbb{L}^{3}$ são pontos umbílicos, então $\Sigma$ é um plano $(H=0)$ ou um hiperbolóide $(H \neq 0)$.

Prova: Seja $p \in \Sigma$ e considere $X(u, v)=x \circ \varphi(u, v)$ uma parametrização de $\Sigma$ numa vizinhança coordenada $V$ conexa contendo $p$.

Como $\Sigma$ é totalmente umbílica, temos que $\forall q \in V, q$ é umbílico. Então para todo $w \in T_{q}(\Sigma), w=a_{1} X_{u}+a_{2} X_{v}$, onde $\left\{X_{u}, X_{v}\right\}$ é uma base de $T_{q} \Sigma$ e para todos $q \in V, a_{i} \in \mathbb{R}$, sabemos que

$$
A(w)=\lambda_{q} w
$$

onde $A$ é o operador de Weingarten e $\lambda_{q}=\lambda(q)$ é a função autovalor de $A$ nos pontos $q$.

Logo, da equação (1.2)

$$
\nabla_{w}^{\circ} N(q)=-\lambda_{q} w
$$

com $N=N(u, v)=N(X(u, v))$ o campo normal unitário de tipo tempo.

Vamos mostrar que a função auto-valor $\lambda=\lambda(q)$ é constante. Para isso, escrevemos

$$
a_{1} N_{u}+a_{2} N_{v}=w(N)=\nabla_{w}^{o} N=-\lambda w=-\lambda\left(a_{1} X_{u}+a_{2} X_{v}\right)
$$




$$
\begin{gathered}
\quad \begin{array}{l}
N_{u}=-\lambda X_{u} \\
N_{v}=-\lambda X_{v}
\end{array} \\
\Rightarrow 0=N_{u v}-N_{v u}=-\lambda_{v} X_{u}-\lambda X_{u v}+\lambda_{u} X_{v}+\lambda X_{v u}=\lambda_{u} X_{v}-\lambda_{v} X_{u}
\end{gathered}
$$

No entanto, $\left\{X_{u}, X_{v}\right\}$ é linearmente independente, portanto $\lambda_{u}=\lambda_{v}=0 \forall q \in V$. Como $V$ é conexo, $\lambda$ é constante em $V$.

Há duas possibilidades para $\lambda: \lambda=0$ ou $\lambda \neq 0$.

Se $\lambda=0$, então $N_{u}=N_{v}=0$ e, portanto, $N$ é constante na vizinhança conexa $V$. Assim, para os pontos $X(u, v)$ em $V$,

$$
\langle X(u, v), N\rangle_{u}=\langle X(u, v), N\rangle_{v}=0
$$

segue que

$$
\langle X(u, v), N\rangle=\text { constante }
$$

Devemos ter portanto $\left\langle X(u, v)-p_{0}, N\right\rangle=0$ para algum $p_{0} \in \Sigma$, ou seja os pontos de $V$ pertencem a algum plano.

Se $\lambda \neq 0$, seja $y=X(u, v)-\frac{1}{\lambda} N(u, v)$. Então

$$
\begin{aligned}
& \left(X(u, v)+\frac{1}{\lambda} N(u, v)\right)_{u}=X_{u}+\frac{1}{\lambda} N_{u}=0 \\
& \left(X(u, v)+\frac{1}{\lambda} N(u, v)\right)_{v}=X_{v}+\frac{1}{\lambda} N_{v}=0
\end{aligned}
$$

Segue que $y$ é constante no conexo $V$ e fazendo $\langle X(u, v)-y, X(u, v)-y\rangle=$ $\left\langle\frac{1}{\lambda} N, \frac{1}{\lambda} N\right\rangle=-\frac{1}{\lambda^{2}}$, concluímos que os pontos de $V$ pertencem a um hiperbolóide.

Para provarmos que estas situações valem em toda a superfície $\Sigma$ e não apenas numa vizinhança conexa $V$, basta usarmos que $\Sigma$ é conexa. Essa prova é a mesma feita em [10] e isso justifica não a repetirmos aqui.

Com isto, quando o bordo é um círculo, podemos enunciar e provar o seguinte resultado de unicidade.

Teorema 2.7.2. As únicas superfícies compactas de tipo espaço imersas no $\mathbb{L}^{3}$ com curvatura média constante e bordo circular são os discos planares e as calotas hiperbólicas. 
Prova: Seja $a$ o vetor unitário de tipo tempo que aponta para o futuro no $\mathbb{L}^{3}$ tal que $\Gamma=S^{1}(r) \subset a^{\perp}$ que é um plano de tipo espaço. Assim $k=\frac{1}{r}$ e área $(\Omega)=\pi r^{2}$, onde $\Omega$ é o domínio limitado por $\Gamma$. A desigualdade integral assegura que

$$
\oint_{\partial \Sigma} \frac{1}{r}\langle\nu, a\rangle^{2} d s \leq 2 H^{2} \pi r^{2} \Rightarrow \oint_{\partial \Sigma}\langle\nu, a\rangle^{2} d s \leq 2 \pi H^{2} r^{3}
$$

Por outro lado, do corolário 2.6.1 sabemos que

$$
\oint_{\partial \Sigma}\langle\nu, a\rangle d s=2 H \text { área }(\Omega)=2 \pi H r^{2}
$$

E do corolário 1.4.1, segue:

$$
\begin{gathered}
\oint_{\partial \Sigma}\langle\nu, a\rangle^{2} d s \cdot \oint_{\partial \Sigma} 1 d s \geq\left(\oint_{\partial \Sigma}\langle\nu, a\rangle d s\right)^{2} \\
\therefore \oint_{\partial \Sigma}\langle\nu, a\rangle^{2} d s \geq \frac{4 \pi^{2} H^{2} r^{4}}{\text { comprimento }(\Gamma)}=\frac{4 \pi^{2} H^{2} r^{4}}{2 \pi r}=2 \pi H^{2} r^{3}
\end{gathered}
$$

Vale então a igualdade do corolário 2.6.5, implicando que as superfícies com bordo circular $\Gamma$ e curvatura média constante $H$ devem ser totalmente umbílicas. Se $H=0$, temos que tais superfícies são discos planares, e se $H \neq 0$, são calotas hiperbólicas, pela proposição 2.7.1.

Alguns autores vêm buscando um resultado correspondente a este no caso Euclideano, envolvendo discos planares e calotas esféricas. Alguns resultados parciais foram obtidos, ora impondo restrições à curvatura média, ora acrescentando hipóteses ao tipo de superfície.

Para que possamos dar uma idéia do que já foi feito neste campo, apoiamo-nos nas referências [7], [9], [16] e [20].

Seja $\Gamma$ uma curva de Jordan numa bola fechada de raio $r$ no $\mathbb{R}^{3}$, e considere o problema de encontrar uma superfície tendo $\Gamma$ como seu bordo e curvatura média $H$. Se $|H| \leq \frac{1}{r}$, já se sabe que existe pelo menos uma superfície do tipo disco com $H$ constante limitada por $\Gamma$. Mas a unicidade não é válida em geral. H. Brezis e J. M. Coron provaram que existem pelo menos duas soluções para cada $H$ satisfazendo $0<|H|<\frac{1}{r}$. A pergunta feita é se elas são as únicas soluções. 
O caso que nos interessa para a comparação com a situação no $\mathbb{L}^{3}$ é aquele em que $\Gamma$ é um círculo de raio $r$. Sabe-se que há duas soluções para o problema: duas calotas esféricas (a grande e a pequena, ou a maior e a menor) limitadas por $\Gamma$, com raio $\frac{1}{|H|}, 0<|H| r<1$ e com curvatura média constante $H$ (ou $-H$ ). Mas mesmo neste caso simples, não sabemos se há outras soluções.

Para o caso de dimensão $n$, o resultado é provado em [16] para hipersuperfícies compactas mergulhadas com curvatura média constante não nula cujo bordo é uma esfera $(n-1)$-dimensional contida num hiperplano, assumindo que as hipersuperfícies não atravessam o hiperplano (não intersectam o lado de fora da esfera). No caso de dimensão dois, a prova é baseada no Método de Reflexão de Alexandrov e na classificação de Delaunay para superfícies de revolução, e pode ser encontrado resumidamente em [9].

Supondo a superfície compacta com curvatura média constante $H$ imersa no espaço Euclideano com bordo contido num plano $P$, prova-se que ela é uma calota esférica na situação em que

$$
h=\frac{H A^{+}}{2 \pi},
$$

onde $A^{+}$é a área da região de $\Sigma$ acima do plano que contém o bordo e $h$ é a altura de $\Sigma$ até esse plano.[20, Teorema 1]

Com a hipótese adicional que a imagem da superfície pela imersão está contida num cilindro sólido fechado de raio $\frac{1}{|H|}$, é possível mostrar que a imersão descreve uma calota esférica [7].

Além disto, temos a fórmula do fluxo no caso Euclideano [ver 19, para mais detalhes], que é usada em alguns artigos para a obtenção dos resultados (parciais) de unicidade. Ela foi obtida por N. Korevaar, R. Kusner e B. Solomon e tem a seguinte formulação:

Lema 2.7.3. Seja $M$ uma superfície compacta com bordo $\partial M$ e $\varphi: M \rightarrow \mathbb{R}^{3}$ uma imersão com curvatura média constante $H$. Denotamos por $\alpha: \partial M \rightarrow \mathbb{R}^{3}$ a restrição de $\varphi$ ao bordo de $M$ e por $N: M \rightarrow \mathbb{R}^{3}$ a aplicação de Gauss de $\varphi$. Então

$$
H \int_{\partial M} \alpha \wedge \alpha^{\prime}=-\int_{\partial M} N \wedge \alpha^{\prime}
$$




\section{Capítulo 3}

\section{O Princípio do Máximo}

Neste capítulo vamos provar um resultado de unicidade para superfícies compactas de tipo espaço com curvatura média constante fazendo o uso do Princípio do Máximo de Hopf, além de darmos uma outra abordagem para a prova do Teorema 2.7.2.

\subsection{Gráficos de tipo espaço}

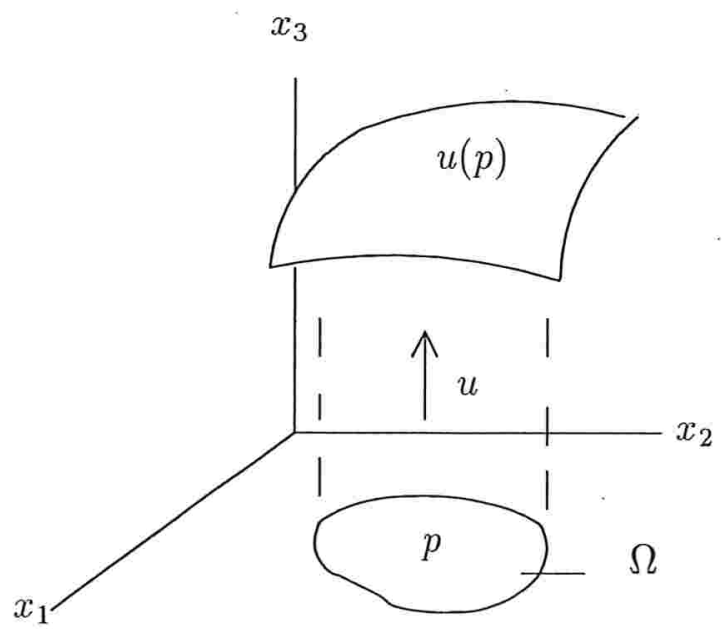

Figura 3.1: Representação de um gráfico

Para cada função diferenciável $u: \bar{\Omega} \rightarrow \mathbb{R}$, com $\bar{\Omega}$ um domínio compacto no $\mathbb{R}^{2}$ 
e $u=u\left(x_{1}, x_{2}\right)$, temos o mergulho natural $X: \bar{\Omega} \rightarrow \mathbb{L}^{3}$ definido por

$$
X\left(x_{1}, x_{2}\right)=\left(x_{1}, x_{2}, u\left(x_{1}, x_{2}\right)\right) .
$$

Proposição 3.1.1. X induz um tensor métrico (não necessariamente não degenerado) sobre $\bar{\Omega}$ que é dado por

$$
\left\langle d X_{p}(v), d X_{p}(w)\right\rangle=\langle v, w\rangle-\langle D u(p), v\rangle\langle D u(p), w\rangle, p \in \bar{\Omega}, v, w \in \mathbb{R}^{2}
$$

Além disto, o gráfico determinado por u é de tipo espaço se e somente se $|D u|<1$ onde Du denota o gradiente de u no $\mathbb{R}^{2}$.

Prova: Para cada $p \in \bar{\Omega}, v, w \in \mathbb{R}^{2}, v=\left(v_{1}, v_{2}\right), w=\left(w_{1}, w_{2}\right)$, temos $d X_{p}(v)=\left[\begin{array}{cc}1 & 0 \\ 0 & 1 \\ \frac{\partial u}{\partial x_{1}}(p) & \frac{\partial u}{\partial x_{2}}(p)\end{array}\right] \cdot\left[\begin{array}{c}v_{1} \\ v_{2}\end{array}\right]=\left(v_{1}, v_{2}, \frac{\partial u}{\partial x_{1}}(p) v_{1}+\frac{\partial u}{\partial x_{2}}(p) v_{2}\right)=\left(v_{1}, v_{2},\langle D u(p), v\rangle\right)$

Analogamente, $d X_{p}(w)=\left(w_{1}, w_{2},\langle D u(p), w\rangle\right)$, onde $D u$ denota o gradiente de $u$ no $\mathbb{R}^{2}$.

Portanto o tensor métrico em $\bar{\Omega}$ é dado por $\left\langle d X_{p}(v), d X_{p}(w)\right\rangle=v_{1} w_{1}+v_{2} w_{2}-\langle D u(p), v\rangle\langle D u(p), w\rangle=\langle v, w\rangle-\langle D u(p), v\rangle\langle D u(p), w\rangle$

O gráfico é de tipo espaço se e somente se $\left\langle d X_{p}(v), d X_{p}(v)\right\rangle \geq 0$ para todo $p \in \bar{\Omega}$ e para todo $v \in \mathbb{R}^{2}$.

Sendo $\theta$ o ângulo entre $D u$ e $v$, temos:

$$
\begin{aligned}
&\left\langle d X_{p}(v), d X_{p}(v)\right\rangle=\langle v, v\rangle-\langle D u(p), v\rangle^{2}= \\
&=\langle v, v\rangle-\langle D u(p), D u(p)\rangle\langle v, v\rangle \cos ^{2} \theta= \\
&=\langle v, v\rangle\left(1-\langle D u(p), D u(p)\rangle \cos ^{2} \theta\right)= \\
&=\langle v, v\rangle\left(1-|D u(p)|^{2} \cos ^{2} \theta\right) \\
& \therefore\left\langle d X_{p}(v), d X_{p}(v)\right\rangle \geq 0 \Leftrightarrow 1-|D u(p)|^{2} \cos ^{2} \theta \geq 0 \Leftrightarrow \\
& \Leftrightarrow|D u(p)|^{2} \cos ^{2} \theta \leq 1 \Leftrightarrow \\
& \Leftrightarrow|D u(p)|^{2} \leq 1 \forall p \in \bar{\Omega} \Leftrightarrow|D u| \leq 1
\end{aligned}
$$


Além disso, a igualdade vale se e somente se $v=0$.

De fato, se $v=0$ então $\left\langle d X_{p}(v), d X_{p}(v)\right\rangle=0$ da sua definição e, por outro lado, se $v \neq 0$, então, do que foi feito anteriormente, $\left\langle d X_{p}(v), d X_{p}(v)\right\rangle=0$ se e só se $1-|D u|^{2} \cos ^{2} \theta=0$. Para tanto, devemos ter $|D u|^{2}=\frac{1}{\cos ^{2} \theta} \geq 1$ e de $|D u| \leq 1$ segue $|D u|=1$.

Mas isso implica que existe solução para $\left\langle d X_{p}(v), d X_{p}(v)\right\rangle=0$ com $v$ não nulo e paralelo a $D u$, o que torna o tensor métrico degenerado e assim ele não pode ser de tipo espaço, o que é uma contradição.

Logo, o gráfico determinado por $u$ é de tipo espaço se e somente se $|D u|<1$.

Na proposição seguinte mostramos que toda superfície compacta de tipo espaço imersa no $\mathbb{L}^{3}$, limitada por uma curva que se projeta sobre uma curva de Jordan plana, pode ser vista como um gráfico de tipo espaço determinado por alguma função diferenciável $u$. Isso é essencial para o resultado que buscamos.

Proposição 3.1.2. Seja $x: \Sigma \rightarrow \mathbb{L}^{3}$ uma superfície compacta de tipo espaço imersa no $\mathbb{L}^{3}$ limitada por uma curva $\Gamma$. Vamos assumir que existe um plano de tipo espaço $\Pi$ tal que a projeção ortogonal $\Gamma^{\prime}$ de $\Gamma$ sobre $\Pi$ é uma curva de Jordan plana. Então existe um difeomorfismo $F: \bar{\Omega} \rightarrow \Sigma$ de um domínio compacto $\bar{\Omega} \subset \mathbb{R}^{2}$ tal que $x \circ F$ é um gráfico de tipo espaço sobre $\bar{\Omega}$.

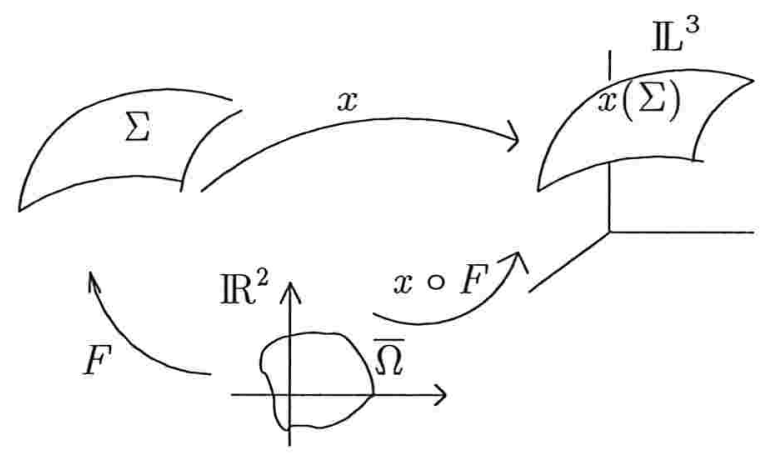

Figura 3.2: Gráfico $x \circ F$

Prova: Começamos assumindo, sem perda de generalidade, que o plano de tipo espaço $\Pi=\mathbb{R}^{2}$ é o plano $\left\{x_{3}=0\right\}$, assim a projeção ortogonal $\pi: \mathbb{L}^{3} \rightarrow \mathbb{R}^{2}$ é a 
projeção definida por $\pi\left(x_{1}, x_{2}, x_{3}\right)=\left(x_{1}, x_{2}, 0\right)$. O que queremos é encontrar a função $F$ e o domínio compacto $\bar{\Omega} \subset \mathbb{R}^{2}$, onde $F$ está definida.

Denotemos por $\tilde{x}$ a projeção $\tilde{x}=\pi \circ x: \Sigma \rightarrow \mathbb{R}^{2}$ e mostremos inicialmente que a aplicação $\tilde{x}_{\mid \operatorname{int}(\Sigma)}: \operatorname{int}(\Sigma) \rightarrow \mathbb{R}^{2}$ é um difeomorfismo local, isto é, $d \tilde{x}_{p}$ é injetora para todo $p$ no $\operatorname{int}(\Sigma)$.

Seja $p \in \operatorname{int}(\Sigma)$ qualquer. Se $u, v \in T_{p} \Sigma \operatorname{com} d \tilde{x}_{p}(u)=d \tilde{x}_{p}(v)$, então

$$
\begin{aligned}
d \pi_{x(p)}\left(d x_{p}(u)\right)=d \pi_{x(p)}\left(d x_{p}(v)\right) & \Rightarrow \pi_{x(p)}\left(u_{1}^{\prime}, u_{2}^{\prime}, u_{3}^{\prime}\right)=\pi_{x(p)}\left(v_{1}^{\prime}, v_{2}^{\prime}, v_{3}^{\prime}\right) \Rightarrow \\
& \Rightarrow\left(u_{1}^{\prime}, u_{2}^{\prime}, 0\right)=\left(v_{1}^{\prime}, v_{2}^{\prime}, 0\right) \Rightarrow u_{1}^{\prime}=v_{1}^{\prime} \text { e } u_{2}^{\prime}=v_{2}^{\prime},
\end{aligned}
$$

onde $d x_{p}(u)=\left(u_{1}^{\prime}, u_{2}^{\prime}, u_{3}^{\prime}\right)$ e $d x_{p}(v)=\left(v_{1}^{\prime}, v_{2}^{\prime}, v_{3}^{\prime}\right)$.

Suponhamos, por absurdo, que $u_{3}^{\prime} \neq v_{3}^{\prime}$ e consideremos a reta $r$ que une $d x_{p}(u)$ a $d x_{p}(v)$ em $T_{p} \Sigma$. Ela é dada por $r: X=\left(x_{1}, x_{2}, x_{3}\right)=\left(u_{1}^{\prime}, u_{2}^{\prime}, u_{3}^{\prime}\right)+\lambda\left(0,0, v_{3}^{\prime}-u_{3}^{\prime}\right)$.

Para $\lambda=\frac{-u_{3}^{\prime} \pm \sqrt{u_{1}^{\prime 2}+u_{2}^{\prime 2}}}{v_{3}^{\prime}-u_{3}^{\prime}}$ temos $r$ atravessando o cone de luz $x_{3}^{2}=x_{1}^{2}+x_{2}^{2}$, o que contradiz a hipótese de $r \in T_{p} \Sigma$, isto é, de $r$ ser de tipo espaço. Desta forma, $u_{3}^{\prime}=v_{3}^{\prime}$ e, portanto, $d x_{p}(u)=d x_{p}(v)$ e como $d x_{p}$ é injetora, segue que $u=v$. Logo $\tilde{x}=\pi \circ x: \operatorname{int}(\Sigma) \rightarrow \mathbb{R}^{2}$ é um difeomorfismo local, e portanto é uma aplicação aberta.

Considere $\Omega=\tilde{x}(\operatorname{int}(\Sigma)) \subset \mathbb{R}^{2}$, que é um subconjunto aberto no $\mathbb{R}^{2}$, e $\Omega^{\prime}$ o domínio planar limitado pela curva de Jordan plana $\Gamma^{\prime}=\pi(\Gamma)=\pi(x(\partial \Sigma))=\tilde{x}(\partial \Sigma)$. O nosso objetivo é mostrar que $\tilde{x}: \Sigma \rightarrow \bar{\Omega}$ é um difeomorfismo global e, assim, escolhendo $F=\tilde{x}^{-1}$ teremos que $x \circ F$ é um gráfico determinado pela função $u=$ $x_{3} \circ F$.

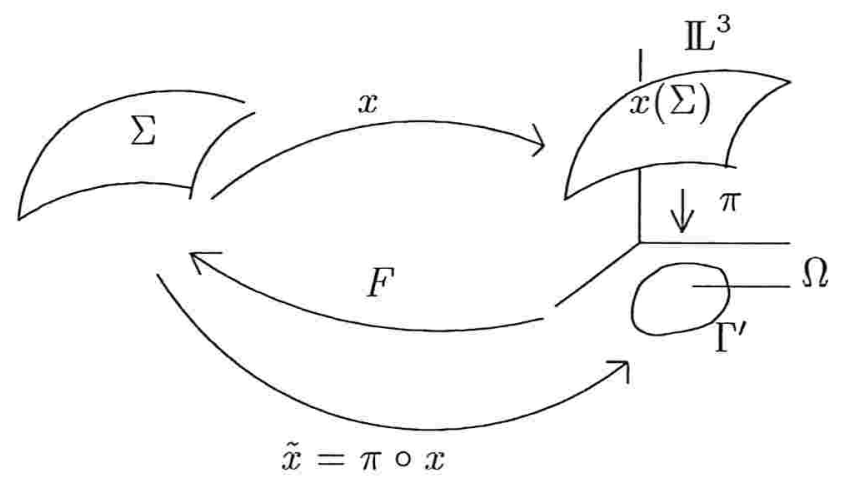

Figura 3.3: Construção de $F$ 
Para isso, vamos mostrar que $\Omega=\Omega^{\prime}$.

Vamos provar primeiro que $\partial \Omega=\partial \tilde{x}(\operatorname{int}(\Sigma))=\partial \tilde{x}(\Sigma) \subset \Gamma^{\prime}=\tilde{x}(\partial \Sigma)$.

Como $\Sigma$ é compacta, para qualquer $q \in \partial \tilde{x}(\Sigma)$ existe $p \in \Sigma$ tal que $\tilde{x}(p)=q$.

Há duas possibilidades para o ponto $p: p \in \operatorname{int}(\Sigma)$ ou $p \in \partial \Sigma$. Vamos mostrar que só pode ocorrer $p \in \partial \Sigma$.

Se $p \in \operatorname{int}(\Sigma)$, já vimos que $\tilde{x}: \operatorname{int}(\Sigma) \rightarrow \mathbb{R}^{2}$ é um difeomorfismo local. Logo existem abertos $U_{p}$ de $p$ em $\operatorname{int}(\Sigma)$ e $V_{q}$ de $q$ em $\Omega$ (pois $\tilde{x}(\operatorname{int}(\Sigma))=\Omega$ ) tais que $\tilde{x}: U_{p} \rightarrow V_{q}$ é um difeomorfismo. Isso faz com que $q \in \Omega$, o que é uma contradição, já que $q \in \partial \Omega$. Logo $p \in \partial \Sigma$. Portanto $\tilde{x}(p)=q \in \Gamma^{\prime}$, ou seja, $\partial \Omega \subset \Gamma^{\prime}$.

Se existe um ponto $p \in \Omega$ que não está em $\Omega^{\prime}$, o fato de $\Omega$ ser limitado nos diz que há uma bola fechada $B$ inteiramente contida em $\Omega$ com centro nesse ponto $p$ que encontra o bordo de $\Omega$. Assim, a distância entre esse ponto $\tilde{p}$ de interseção e $\Gamma^{\prime}$ é maior que a distância de $p$ a $\Gamma^{\prime}\left(d\left(\tilde{p}, \Gamma^{\prime}\right)=d(\tilde{p}, p)+d\left(p, \Gamma^{\prime}\right)\right)$. Logo há pontos em $\partial \Omega$ fora de $\Gamma^{\prime}$, o que não é possível. Portanto, temos $\Omega \subset \Omega^{\prime}$.

Analogamente, se existe um ponto em $\Omega^{\prime}$ que não está em $\Omega$, há pontos em $\partial \Omega$ dentro de $\Omega^{\prime}$, o que novamente não é possível, e segue que $\Omega^{\prime} \subset \Omega$.
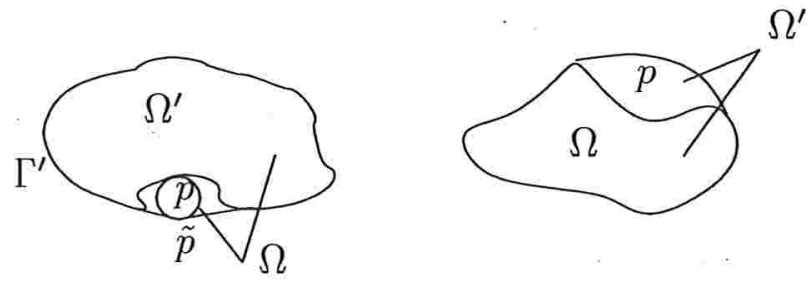

Figura 3.4: Domínios $\Omega$ e $\Omega^{\prime}$

Com isso provamos que $\Omega=\Omega^{\prime}$. Como conseqüência $\tilde{x}: \Sigma \rightarrow \bar{\Omega}$ é um difeomorfismo local e a compacidade de $\Sigma$ junto com a conexidade de $\bar{\Omega}$ implicam que $\tilde{x}$ é uma aplicação de recobrimento. Como $\Omega$ é simplesmente conexo (domínio limitado por curva de Jordan), $\tilde{x}$ é um homeomorfismo. Portanto $\tilde{x}$ é um difeomorfismo.

Segue, trivialmente, a seguinte conseqüência: 
Corolário 3.1.3. Toda superfície compacta de tipo espaço imersa no $\mathbb{L}^{3}$ limitada por uma curva de Jordan plana é um gráfico de tipo espaço.

Em [24] encontramos um resultado similar à Proposição 3.1.2, com a hipótese de que a superfície é completa. Prova-se, neste caso, que a superfície é um gráfico sobre todo $\mathbb{R}^{2}$.

O objetivo, agora, é provar que uma superfície dada como acima, que tem curvatura média constante, está bem definida, isto é, dados o seu bordo e a constante $H$, que é a sua curvatura média, ela é única. Isso envolve a teoria de equações diferenciais parciais elípticas e o Princípio do Máximo de Hopf que veremos mais adiante.

\subsection{A equação da curvatura média}

Seja $\Omega$ um domínio no $\mathbb{R}^{2}$ e $u$ uma função de classe $C^{2}$ em $\Omega$ e contínua em $\bar{\Omega}$, $u: \Omega \subset \mathbb{R}^{2} \rightarrow \mathbb{R}$.

Proposição 3.2.1. Se u define um gráfico de tipo espaço sobre $\Omega$, então a função curvatura média $H$ do gráfico é dada por

$$
\operatorname{Div}\left(\frac{D u}{\sqrt{1-|D u|^{2}}}\right)=2 H
$$

onde Div é o divergente no $\mathbb{R}^{2}$.

Prova: Seja $X\left(x_{1}, x_{2}\right)=\left(x_{1}, x_{2}, u\left(x_{1}, x_{2}\right)\right)$ o gráfico de tipo espaço determinado por $u$.

Vamos calcular a função curvatura média $H$ deste gráfico. Os coeficientes da primeira forma fundamental de $X(\Omega)$ são $E=1-\left(\frac{\partial u}{\partial x_{1}}\right)^{2}, F=-\frac{\partial u}{\partial x_{1}} \frac{\partial u}{\partial x_{2}} e G=$ $1-\left(\frac{\partial u}{\partial x_{2}}\right)^{2}$.

$\operatorname{Temos} X_{x_{1}} \wedge X_{x_{2}}=\left(-\frac{\partial u}{\partial x_{1}},-\frac{\partial u}{\partial x_{2}},-1\right) e\left\|X_{x_{1}} \wedge X_{x_{2}}\right\|=\sqrt{1-|D u|^{2}}$, pois $|D u|<1$.

Escolhemos $\mathrm{N}$ apontando para o futuro $\left(x_{3}>0\right)$

$$
\therefore N=-\frac{X_{x_{1}} \wedge X_{x_{2}}}{\left\|X_{x_{1}} \wedge X_{x_{2}}\right\|}=\frac{1}{\sqrt{1-|D u|^{2}}}\left(\frac{\partial u}{\partial x_{1}}, \frac{\partial u}{\partial x_{2}}, 1\right)
$$


Logo, de $H=-\frac{1}{2} \frac{e G-2 f F+g E}{E G-F^{2}}$ temos:

$$
\begin{aligned}
& H=\frac{1}{2} \frac{\frac{\partial^{2} u}{\partial x_{1}^{2}}\left(1-\left(\frac{\partial u}{\partial x_{2}}\right)^{2}\right)+2 \frac{\partial^{2} u}{\partial x_{1} x_{2}} \frac{\partial u}{\partial x_{1}} \frac{\partial u}{\partial x_{2}}+\left(1-\left(\frac{\partial u}{\partial x_{1}}\right)^{2}\right) \frac{\partial^{2} u}{\partial x_{2}^{2}}}{\left[\left(1-\left(\frac{\partial u}{\partial x_{1}}\right)^{2}\right)\left(1-\left(\frac{\partial u}{\partial x_{2}}\right)^{2}\right)-\left(\frac{\partial u}{\partial x_{1}}\right)^{2}\left(\frac{\partial u}{\partial x_{2}}\right)^{2}\right]\left[1-\left(\frac{\partial u}{\partial x_{1}}\right)^{2}-\left(\frac{\partial u}{\partial x_{2}}\right)^{2}\right]^{\frac{1}{2}}}= \\
& =\frac{1}{2} \frac{\left(1-\left(\frac{\partial u}{\partial x_{2}}\right)^{2}\right) \frac{\partial^{2} u}{\partial x_{1}^{2}}+\left(1-\left(\frac{\partial u}{\partial x_{1}}\right)^{2}\right) \frac{\partial^{2} u}{\partial x_{2}^{2}}+2 \frac{\partial u}{\partial x_{1}} \frac{\partial u}{\partial x_{2}} \frac{\partial^{2} u}{\partial x_{1} x_{2}}}{\left(1-\left(\frac{\partial u}{\partial x_{1}}\right)^{2}-\left(\frac{\partial u}{\partial x_{2}}\right)^{2}\right)^{\frac{3}{2}}}
\end{aligned}
$$

Por outro lado

$$
\begin{gathered}
\operatorname{Div}\left(\frac{D u}{\sqrt{1-|D u|^{2}}}\right)=\operatorname{Div}\left(\frac{\frac{\partial u}{\partial x_{1}}}{\sqrt{1-\left(\frac{\partial u}{\partial x_{1}}\right)^{2}-\left(\frac{\partial u}{\partial x_{2}}\right)^{2}}}, \frac{\frac{\partial u}{\partial x_{2}}}{\sqrt{1-\left(\frac{\partial u}{\partial x_{1}}\right)^{2}-\left(\frac{\partial u}{\partial x_{2}}\right)^{2}}}\right)= \\
=\frac{\frac{\partial^{2} u}{\partial x_{1}^{2}}\left(\sqrt{1-|D u|^{2}}\right)-\frac{\partial u}{\partial x_{1}} \cdot \frac{-2 \frac{\partial u}{\partial x_{1}} \frac{\partial^{2} u}{\partial x_{1}^{2}}-2 \frac{\partial u}{\partial x_{2}} \frac{\partial^{2} u}{\partial x_{1} \partial x_{2}}}{2 \sqrt{1-|D u|^{2}}}+\frac{\partial^{2} u}{\partial x_{2}^{2}}\left(\sqrt{1-|D u|^{2}}\right)-\frac{\partial u}{\partial x_{2}} \cdot \frac{-2 \frac{\partial u}{\partial x_{1}} \frac{\partial^{2} u}{\partial x_{2} \partial x_{1}}-2 \frac{\partial u}{\partial x_{2}} \frac{\partial^{2} u}{\partial x_{2}^{2}}}{2 \sqrt{1-|D u|^{2}}}}{1-|D u|^{2}}= \\
=\frac{\frac{\partial^{2} u}{\partial x_{1}^{2}}\left(1-\left(\frac{\partial u}{\partial x_{1}}\right)^{2}-\left(\frac{\partial u}{\partial x_{2}}\right)^{2}\right)+\left(\frac{\partial u}{\partial x_{1}}\right)^{2} \frac{\partial^{2} u}{\partial x_{1}^{2}}+\frac{\partial u}{\partial x_{1}} \frac{\partial u}{\partial x_{2}} \frac{\partial^{2} u}{\partial x_{1} \partial x_{2}}}{\left(1-|D u|^{2}\right)^{\frac{3}{2}}}+ \\
+\frac{\frac{\partial^{2} u}{\partial x_{2}^{2}}\left(1-\left(\frac{\partial u}{\partial x_{1}}\right)^{2}-\left(\frac{\partial u}{\partial x_{2}}\right)^{2}\right)+\left(\frac{\partial u}{\partial x_{2}}\right)^{2} \frac{\partial^{2} u}{\partial x_{2}^{2}}+\frac{\partial u}{\partial x_{1}} \frac{\partial u}{\partial x_{2}} \frac{\partial^{2} u}{\partial x_{1} \partial x_{2}}}{\left(1-|D u|^{2}\right)^{\frac{3}{2}}}= \\
=\frac{\frac{\partial^{2} u}{\partial x_{1}^{2}}\left(1-\left(\frac{\partial u}{\partial x_{2}}\right)^{2}\right)+2 \frac{\partial u}{\partial x_{1}} \frac{\partial u}{\partial x_{2}} \frac{\partial^{2} u}{\partial x_{1} x_{2}}+\frac{\partial^{2} u}{\partial x_{2}^{2}}\left(1-\left(\frac{\partial u}{\partial x_{1}}\right)^{2}\right)}{\left(1-|D u|^{2}\right)^{\frac{3}{2}}}=2 H
\end{gathered}
$$

Portanto, o gráfico determinado por $u$ é uma superfície de tipo espaço com curvatura média constante $H$ se e só se satisfaz

$$
\left(1-\left(\frac{\partial u}{\partial x_{2}}\right)^{2}\right) \frac{\partial^{2} u}{\partial x_{1}^{2}}+2 \frac{\partial u}{\partial x_{1}} \frac{\partial u}{\partial x_{2}} \frac{\partial^{2} u}{\partial x_{1} \partial x_{2}}+\frac{\partial^{2} u}{\partial x_{2}^{2}}\left(1-\left(\frac{\partial u}{\partial x_{1}}\right)^{2}\right)-2 H\left(1-|D u|^{2}\right)^{\frac{3}{2}}=0
$$

A fim de continuarmos o nosso estudo, vamos enunciar alguns conceitos da teoria de equações elípticas e operadores quasilineares. 


\subsection{Operadores Quasilineares}

Definição 3.3.1. Um operador quasilinear diferencial parcial de $2^{\underline{a}}$ ordem é um operador da forma

$$
Q u=\sum_{i, j} a_{i j}(x, u, D u) \frac{\partial^{2} u}{\partial x_{i} \partial x_{j}}+b(x, u, D u)
$$

onde Du é o gradiente de u.

Considere os operadores quasilineares daquela forma, com $a_{i j}=a_{j i}$, onde $x=$ $\left(x_{1}, x_{2}\right) \in \Omega$ domínio do $\mathbb{R}^{2}$ e $u \in C^{2}(\Omega)$.

Assumimos que os coeficientes $a_{i j}(x, z, p), i, j=1,2, b(x, z, p)$ são definidos para todos os valores de $(x, z, p)$ no conjunto $\Omega \times \mathbb{R} \times \mathbb{R}^{2}$ ( $z$ substitui $u$ e $p$ substitui $D u$ ).

Seja $\mathcal{U}$ um subconjunto de $\Omega \times \mathbb{R} \times \mathbb{R}^{2}$. Então $Q$ é elíptico em $\mathcal{U}$ se a matriz dos coeficientes $\left[a_{i j}(x, z, p)\right]$ é definida positiva para todo $(x, z, p) \in \mathcal{U}$. Isso equivale a dizer que se $\lambda(x, z, p), \Lambda(x, z, p)$ denotam, respectivamente, o mínimo e o máximo autovalores de $\left[a_{i j}(x, z, p)\right]$ então

$$
0<\lambda(x, z, p)|\zeta|^{2} \leq \sum_{i, j} a_{i j}(x, z, p) \zeta_{i} \zeta_{j} \leq \Lambda(x, z, p)|\zeta|^{2}
$$

$\forall \zeta=\left(\zeta_{1}, \zeta_{2}\right) \in \mathbb{R}^{2}-\{0\}$ e $\forall(x, z, p) \in \mathcal{U}$

Se $Q$ é elíptico e o quociente $\frac{\Lambda}{\lambda}$ é limitado, então $Q$ é dito uniformemente elíptico.

Se $Q$ é elíptico em todo conjunto $\Omega \times \mathbb{R} \times \mathbb{R}^{2}$ dizemos que $Q$ é elíptico em $\Omega$.

Agora vamos supor que o operador $Q$ é da forma $Q u=\sum a_{i j}(D u) \frac{\partial^{2} u}{\partial x_{i} \partial x_{j}}+b(D u)$.

Sejam $u, v: \Omega \rightarrow \mathbb{R}$, pelo menos de classe $C^{2}$, satisfazendo $Q u \geq Q v$ em $\Omega$ onde

(i) o operador $Q$ é localmente uniformemente elíptico com respeito a $u$ ou a $v$;

(ii) os coeficientes $a_{i j}, b$ são continuamente diferenciáveis em $\Omega \times \mathbb{R}^{2}$.

Então, supondo $Q$ localmente uniformemente elíptico com respeito a $u$

$$
\begin{aligned}
& 0 \leq Q u-Q v=\sum_{i, j=1}^{2} a_{i j}(D u) \frac{\partial^{2} u}{\partial x_{i} \partial x_{j}}-\sum_{i, j=1}^{2} a_{i j}(D v) \frac{\partial^{2} v}{\partial x_{i} \partial x_{j}}+b(D u)-b(D v)= \\
& =\sum_{i, j=1}^{2} a_{i j}(D u) \frac{\partial^{2}(u-v)}{\partial x_{i} \partial x_{j}}+\sum_{i, j=1}^{2}\left[a_{i j}(D u)-a_{i j}(D v)\right] \frac{\partial^{2} v}{\partial x_{i} \partial x_{j}}+b(D u)-b(D v)
\end{aligned}
$$


Assim, escrevendo

$$
\begin{gathered}
w=u-v \\
c_{i j}(x)=a_{i j}(D u) \\
\sum_{i, j=1}^{2}\left[a_{i j}(D u)-a_{i j}(D v)\right] \frac{\partial^{2} v}{\partial x_{i} \partial x_{j}}+b(D u)-b(D v)=\sum_{k=1}^{2} d_{k} \frac{\partial w}{\partial x_{k}} \\
L w=Q u-Q v
\end{gathered}
$$

e sendo $u_{t}=(1-t) v+t u$ um segmento de $v$ até $u, \operatorname{com} \frac{d}{d t} u_{t}=u-v$ e $\frac{d}{d t} D u_{t}=D u-D v$ temos

$$
\begin{gathered}
0 \leq L w=\sum_{i, j=1}^{2} c_{i j}(x) \frac{\partial^{2} w}{\partial x_{i} \partial x_{j}}+\sum_{k=1}^{2} d_{k} \frac{\partial w}{\partial x_{k}} \text { onde } \\
d_{k}=\sum_{i, j=1}^{2}\left\{\int_{0}^{1} \frac{\partial a_{i j}}{\partial u_{k}}\left(D u_{t}\right) d t\right\} \frac{\partial^{2} v}{\partial x_{i j}}+\int_{0}^{1} \frac{\partial b}{\partial u_{k}}\left(D u_{t}\right) d t .
\end{gathered}
$$

Essa expressão vem de

$$
\begin{aligned}
& a_{i j}(D u)-a_{i j}(D v)=\int_{0}^{1} \cdot \frac{d}{d t}\left(a_{i j}\left(D u_{t}\right)\right) d t=\int_{0}^{1} \sum_{k=1}^{2} \frac{\partial a_{i j}}{\partial u_{k}}\left(D u_{t}\right) \cdot \frac{d}{d t}\left(\left(D u_{t}\right)_{k}\right) d t= \\
&= \int_{0}^{1} \sum_{k=1}^{2} \frac{\partial a_{i j}}{\partial u_{k}}\left(D u_{t}\right)(D u-D v)_{k} d t=\int_{0}^{1} \sum_{k=1}^{2} \frac{\partial a_{i j}}{\partial u_{k}}\left(D u_{t}\right) d t \frac{\partial w}{\partial x_{k}} \\
&=\int_{0}^{1} \sum_{k=1}^{2} \frac{\partial b}{\partial u_{k}}\left(D u_{t}\right) \cdot \frac{d}{d t}\left(\left(D u_{t}\right)_{k}\right) d t=\int_{0}^{1} \sum_{k=1}^{2} \frac{\partial b}{\partial u_{k}}\left(D u_{t}\right) d t \frac{\partial w}{\partial x_{k}}
\end{aligned}
$$

Note que fica definido um operador $L$ que herda a elipticidade de $Q$ e cujos coeficientes também são contínuos. Além disto, a equação (3.5) mostra que se $u$ e $v$ satisfazem $Q u \geq Q v$ então $u-v$ satisfaz uma equação diferencial parcial linear $L(u-v) \geq 0$, onde $L$ é um operador linear diferencial parcial, que estudamos a seguir. 


\section{Equações diferenciais parciais lineares de $2^{a}$ ordem}

Seja $L$ um operador linear diferencial parcial de $2^{\underline{a}}$ ordem da forma

$$
L u(x)=\sum_{i, j=1}^{2} a_{i j}(x) \frac{\partial^{2} u}{\partial x_{i} \partial x_{j}}(x)+\sum_{k=1}^{2} b_{k}(x) \frac{\partial u}{\partial x_{k}}(x), x \in \Omega
$$

onde os coeficientes $a_{i j}=a_{j i}$ e $b_{k}$ são funções contínuas em $\Omega \subset \mathbb{R}^{2}$.

Dizemos que $L$ é elíptico em $\Omega$ se a forma quadrática $Q L(\zeta, \zeta)=\sum a_{i j} \zeta_{i} \zeta_{j}$ é definida positiva em $\Omega$. Analogamente ao caso de operadores quasilineares, isso equivale a dizer que se $\lambda(x), \Lambda(x)$ denotam, respectivamente, o mínimo e o máximo autovalores de $\left[a_{i j}(x)\right]$ então

$$
0<\lambda(x)|\zeta|^{2} \leq \sum_{i, j} a_{i j}(x) \zeta_{i} \zeta_{j} \leq \Lambda(x)|\zeta|^{2}
$$

$L$ é uniformemente elíptico em $\Omega$ se $\frac{\Lambda}{\lambda}$ é limitado em $\Omega$.

Notamos que se os coeficientes $a_{i j}, b_{i}$ são contínuos em $\Omega$, então em qualquer subdomínio limitado $\Omega^{\prime} \subset \subset \Omega, L$ é uniformemente elíptico.

Associada a $L$, temos a equação diferencial parcial linear $L u=0$. Tal equação é dita elíptica se e somente se o operador $L$ é elíptico e, conseqüentemente, não depende de $u$.

\subsection{O Princípio do Máximo de Hopf}

O Princípio do Máximo de Hopf tem um papel importante na Geometria e na Análise. Para a Geometria, ele é bastante utilizado para a busca de resultados de unicidade de hipersuperfícies. O Método de Reflexão de Alexandrov, por exemplo, que pode ser encontrado em [2] e [3] utiliza o Princípio do Máximo para encontrar propriedades características da esfera.

Nesta seção, optamos pelas versões deste teorema escritas por [6] e [18], que são trabalhos sobre hipersuperfícies com curvatura média constante, por ser mais simples a sua aplicação no nosso contexto. 
Teorema 3.4.1. (a)(Princípio do Máximo no interior) Suponha que a função u(x) satisfaça a desigualdade $L u \geq 0$, com $L$ um operador linear uniformemente elíptico em $\Omega$. Se u atinge o seu máximo $M$ num ponto interior de $\Omega$, então $u \equiv M$ em $\Omega$. (b)(Princípio do Máximo no bordo) Seja $u(x)$ satisfazendo $L u \geq 0$, com $L$ um operador linear uniformemente elíptico num dominio $\Omega$ com bordo diferenciável $\partial \Omega$. Se $u$ atinge o seu máximo $M$ num ponto de bordo $x_{0}$ onde Du existe e $\frac{\partial u}{\partial \eta}\left(x_{0}\right)=0$, onde $\eta$ é o normal exterior a $\partial \Omega$ em $x_{0}$, então $u \equiv M$ em $\Omega$.

Este teorema é a adaptação do Lema de Hopf e do Princípio do Máximo forte que aparecem em [12].

\subsection{O segundo resultado de unicidade}

Sejam $\Omega$ o domínio onde a função $u$ que determina um gráfico de tipo espaço está definida e $Q$ o operador definido através da equação (3.2)

$$
Q u=\left(1-|D u|^{2}\right) \sum_{i=1}^{2} \frac{\partial^{2} u}{\partial x_{i}^{2}}+\sum_{i, j=1}^{2} \frac{\partial u}{\partial x_{i}} \frac{\partial u}{\partial x_{j}} \frac{\partial^{2} u}{\partial x_{i} \partial x_{j}}-2 H\left(1-\left|D u^{2}\right|\right)^{\frac{3}{2}}=0
$$

com $|D u|<1$ sobre o domínio $\Omega$.

Observamos que o operador $Q$ é quasilinear, já que é escrito da forma (3.3).

Lema 3.5.1. O operador $Q$ é elíptico.

Prova: Para vermos isso, considere a matriz dos coeficientes $\left[a_{i j}(x)\right]$, onde $a_{11}=$ $1-\left(\frac{\partial u}{\partial x_{2}}\right)^{2}, a_{12}=a_{21}=\frac{\partial u}{\partial x_{1}} \frac{\partial u}{\partial x_{2}}$ e $a_{22}=1-\left(\frac{\partial u}{\partial x_{1}}\right)^{2}$. Mostrar que essa matriz é definida positiva é mostrar que

$$
\sum_{i, j=1}^{2} a_{i j} \lambda_{i} \lambda_{j} \geq 0 \text { e a igualdade vale se e somente se } \lambda_{i}=0, i=1,2
$$

Seja $\lambda=\left(\lambda_{1}, \lambda_{2}\right) \in \mathbb{R}^{2}-\{0\}$ qualquer então (3.7) fica escrita como

$$
\sum_{i, j=1}^{2} a_{i j} \lambda_{i} \lambda_{j}=\left(1-\left(\frac{\partial u}{\partial x_{2}}\right)^{2}\right) \lambda_{1}^{2}+2 \frac{\partial u}{\partial x_{1}} \frac{\partial u}{\partial x_{2}} \lambda_{1} \lambda_{2}+\left(1-\left(\frac{\partial u}{\partial x_{1}}\right)^{2}\right) \lambda_{2}^{2}
$$


Agora note que $|D u|<1$ implica $1-\left(\frac{\partial u}{\partial x_{1}}\right)^{2}-\left(\frac{\partial u}{\partial x_{2}}\right)^{2}>0$. Logo

$$
1-\left(\frac{\partial u}{\partial x_{2}}\right)^{2}>\left(\frac{\partial u}{\partial x_{1}}\right)^{2} \geq 0 .
$$

Portanto existe $\varepsilon>0$ tal que $1-\left(\frac{\partial u}{\partial x_{2}}\right)^{2}=\varepsilon+\left(\frac{\partial u}{\partial x_{1}}\right)^{2}$.

Analogamente, existe $\delta>0$ tal que $1-\left(\frac{\partial u}{\partial x_{1}}\right)^{2}=\delta+\left(\frac{\partial u}{\partial x_{2}}\right)^{2}$.

Logo

$$
\begin{aligned}
\sum_{i, j=1}^{2} a_{i j} \lambda_{i} \lambda_{j} & =\left(\varepsilon+\left(\frac{\partial u}{\partial x_{1}}\right)^{2}\right) \lambda_{1}^{2}+2 \frac{\partial u}{\partial x_{1}} \frac{\partial u}{\partial x_{2}} \lambda_{1} \lambda_{2}+\left(\delta+\left(\frac{\partial u}{\partial x_{2}}\right)^{2}\right) \lambda_{2}^{2}= \\
& =\varepsilon \lambda_{1}^{2}+\delta \lambda_{2}^{2}+\left(\frac{\partial u}{\partial x_{1}}\right)^{2} \lambda_{1}^{2}+2 \frac{\partial u}{\partial x_{1}} \frac{\partial u}{\partial x_{2}} \lambda_{1} \lambda_{2}+\left(\frac{\partial u}{\partial x_{2}}\right)^{2} \lambda_{2}^{2}= \\
& =\varepsilon \lambda_{1}^{2}+\delta \lambda_{2}^{2}+\left(\frac{\partial u}{\partial x_{1}} \lambda_{1}+\frac{\partial u}{\partial x_{2}} \lambda_{2}\right)^{2} .
\end{aligned}
$$

Temos

$\sum_{i, j=1}^{2} a_{i j} \lambda_{i} \lambda_{j}=0 \Leftrightarrow \varepsilon \lambda_{1}^{2}=0, \delta \lambda_{2}^{2}=0 e\left(\frac{\partial u}{\partial x_{1}} \lambda_{1}+\frac{\partial u}{\partial x_{2}} \lambda_{2}\right)^{2}=0 \Leftrightarrow \lambda_{1}=\lambda_{2}=0$.

$\operatorname{Mas} \lambda=\left(\lambda_{1}, \lambda_{2}\right) \neq 0$.

Portanto, a matriz $\left[a_{i j}(x)\right]$ é definida positiva e $Q$ é um operador quasilinear elíptico.

Outra forma de mostrarmos que $Q$ é elíptico é notando que

$$
\left(1-|D u|^{2}\right)|\zeta|^{2} \leq \sum_{i, j=1}^{2} a_{i j} \zeta_{i} \zeta_{j} \leq|\zeta|^{2},
$$

pois $\lambda=(1-\mid D u)^{2}$ é o mínimo autovalor e $\Lambda=1$ é o máximo autovalor de $\left[a_{i j}(x)\right]$.

Entretanto $Q$ não é uniformemente elíptico pois $\frac{\Lambda}{\lambda}=\frac{1}{1-|D u|^{2}}$ e $0<1-|D u|^{2}<1$. Mas $Q$ é localmente uniformemente elíptico, o que é importante para usarmos o que foi feito em (3.5).

Vimos também que se $u$ e $v$ são funções definidas num domínio compacto $\bar{\Omega}$ do $\mathbb{R}^{2}$ satisfazendo a equação (3.6) então, de (3.5) $u-v$ satisfaz uma equação elíptica linear $L(u-v)=0$ para a qual vamos aplicar o Princípio do Máximo de Hopf a fim de obtermos o seguinte resultado de unicidade. 
Teorema 3.5.2. Sejam $\Sigma_{1}$ e $\Sigma_{2}$ duas superfícies compactas de tipo espaço com curvatura média constante imersas no $\mathbb{L}^{3}$ limitadas por uma curva que se projeta sobre uma curva de Jordan plana contida num plano de tipo espaço. Se elas têm a mesma curvatura média para uma orientação comum então $\Sigma_{1}=\Sigma_{2}$.

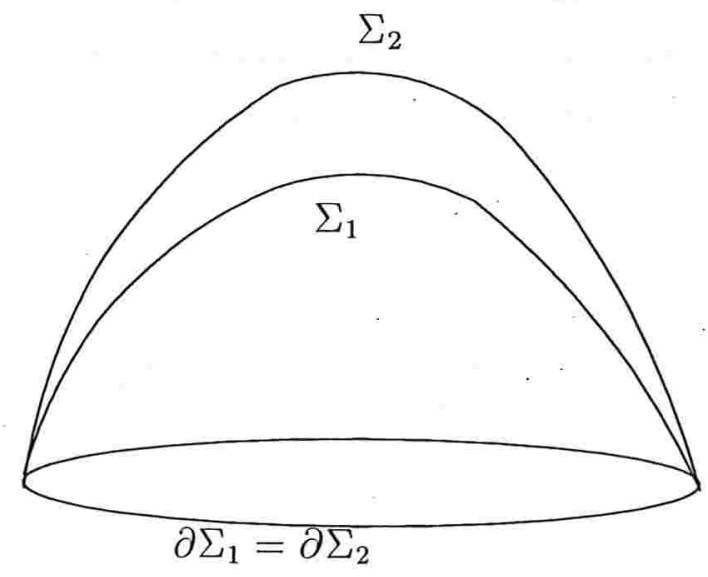

Figura 3.5: Representação de $\Sigma_{1}$ e $\Sigma_{2}$

Prova: Como $\Sigma_{1}$ e $\Sigma_{2}$ são limitadas por uma curva que se projeta sobre uma curva de Jordan plana, temos da Proposição 3.1.2 que podemos parametrizá-las, globalmente, como gráficos de tipo espaço determinados por funções diferenciáveis $u, v: \bar{\Omega} \rightarrow \mathbb{R}^{2} \operatorname{com} \Omega$ o domínio planar limitado pela curva de Jordan que está contida num plano de tipo espaço, e satisfazendo $|D u|<1,|D v|<1$. Supomos $u$ como o gráfico de $\Sigma_{1}$ e $v$ como o gráfico de $\Sigma_{2}$. Além disto, supomos que $\Sigma_{2}$ está acima de $\Sigma_{1}$, isto é, $u \leq v$ em $\bar{\Omega}$. Isso faz com que $u-v$ atinja seu máximo 0 nos pontos de bordo $\partial \Omega$. Sabemos, do fato de $u$ e $v$ possuirem mesma orientação e mesma curvatura média, que ambas satisfazem a equação quasilinear e elíptica (3.6), portanto, $u-v$ satisfaz uma equação elíptica linear $L(u-v)=0$. As funções $u$ e $v$ estão definidas em um domínio compacto, logo os autovalores de $\left[a_{i j}(x)\right]$ de $L$ são limitados, o que torna $L$ uniformemente elíptico. Por outro lado, a derivada de $u-v$ na direção do normal exterior ao bordo nos pontos de bordo é zero, pois os bordos são iguais e portanto tangentes. Pelo Princípio do Máximo (b), a função $u-v$ é identicamente nula em $\Omega$, ou seja, $u \equiv v$ em $\bar{\Omega}$. Segue que $\Sigma_{1}=\Sigma_{2}$. 
Observação 3.5.3. Na prova do teorema 3.5.2, poderíamos ter suposto que $\Sigma_{2}$ está apenas localmente acima de $\Sigma_{1}$. Assim, consideraríamos as funções $u$ e $v$ definidas num subconjunto de $\bar{\Omega}$ contendo parte de seu bordo $\partial \Omega$, onde elas coincidem. Isso faria com que o problema ficasse dividido em duas situações: se $u \leq v\left(\Sigma_{2}\right.$ acima de $\left.\Sigma_{1}\right)$ e se $u \geq v\left(\Sigma_{1}\right.$ acima de $\left.\Sigma_{2}\right)$. Não estaríamos excluindo, portanto, que $\Sigma_{1}$ e $\Sigma_{2}$ tenham outros pontos comuns além dos pontos de bordo. Em qualquer caso, bastaria aplicar o Princípio do Máximo para cada um desses subconjuntos de $\bar{\Omega}$.

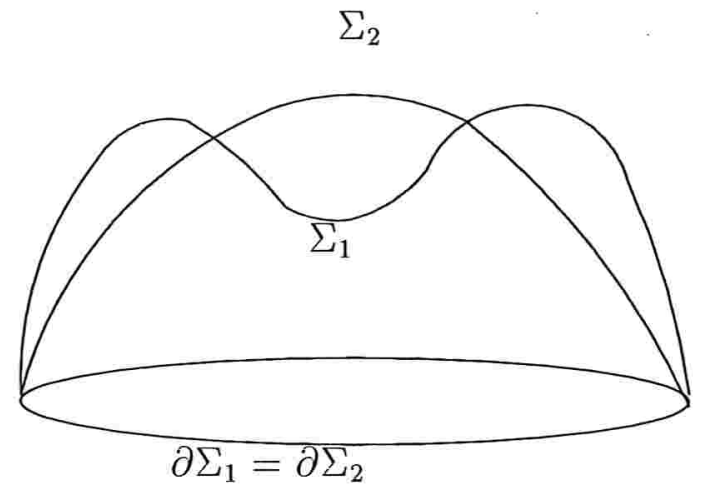

Figura 3.6: Caso geral para o teorema 3.5.2

Com o Teorema 3.5.2, podemos dar uma outra prova para o Teorema 2.7 .2 baseada na teoria de equações elípticas. Isso porque, dado que o bordo de $\Sigma$ é um círculo, e sendo $H$ a curvatura média, teremos apenas duas possibilidades para $\Sigma$ :

- Se $H=0$, comparamos a superfície compacta de tipo espaço com curvatura média constante imersa no $\mathbb{L}^{3}$ com um disco planar limitado por este círculo com mesma orientação que $\Sigma$. O Teorema acima implica que $\Sigma$ é um disco planar.

- Se $H \neq 0$, comparamos a superfície compacta de tipo espaço com curvatura média constante imersa no $\mathbb{L}^{3}$ com uma calota hiperbólica limitada por este círculo com mesma orientação que $\Sigma$. Do Teorema 3.5.1 segue que $\Sigma=$ $\left\{\left(x_{1}, x_{2}, x_{3}\right) \in \mathbb{L}^{3} / x_{1}^{2}+x_{2}^{2}-x_{3}^{2}=-\frac{1}{H^{2}}, 0<x_{3} \leq \sqrt{r^{2}+\frac{1}{H^{2}}}\right\}$, onde $r$ é o raio do círculo. 
Conseqüentemente, as únicas superfícies compactas de tipo espaço com curvatura média constante, imersas no $\mathbb{L}^{3}$, cujo bordo é um círculo, são os discos planares e as calotas hiperbólicas.

\subsection{O Princípio da Tangência}

Antes de encerrarmos este capítulo, pretendemos deixar registrado um resultado encontrado em [4], onde ele foi utilizado para a prova do Teorema 3.5.2, que é chamado de Princípio do Máximo, ou simplesmente, o Princípio da Tangência, como encontrado em [17] e [18]. Em nosso trabalho, optamos pelo Princípio do Máximo clássico, apenas pelo fato de acharmos que esta prova seria mais construtiva e rápida, ao invés daquela sugerida no artigo-base da dissertação.

Pretendemos aqui estudar brevemente o Princípio da Tangência.

Toda superfície de tipo espaço com curvatura média constante satisfaz, localmente, uma equação elíptica, que é a equação da curvatura média. Isso porque, localmente, sempre podemos parametrizar uma superfície como um gráfico. Através do Princípio do Máximo de Hopf, podemos provar coincidência local entre duas superfícies sabendo que elas tangenciam-se em algum ponto, já que isso faz com que possamos parametrizá-las como gráficos sobre um mesmo domínio que está contido no plano tangente comum.

Definição 3.6.1. $p \in \Sigma_{1} \cap \Sigma_{2}$ é dito um ponto de tangência entre $\Sigma_{1}$ e $\Sigma_{2}$ se $T_{p} \Sigma_{1}=$ $T_{p} \Sigma_{2}$.

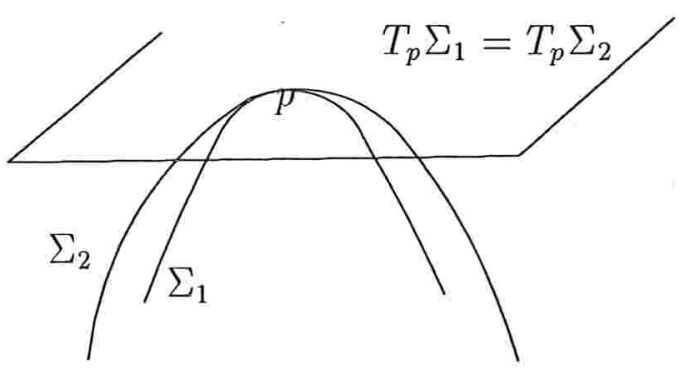

Figura 3.7: O Princípio da tangência 
Lema 3.6.2 (Princípio da Tangência). (a)(ponto no interior) Sejam $\Sigma_{1}$ e $\Sigma_{2}$ duas superfícies de tipo espaço com curvatura média constante. Se $\Sigma_{1}$ e $\Sigma_{2}$ são tangentes em um ponto $p$ e suas curvaturas médias coincidem para uma orientação comum em $p$ e se uma delas está localmente acima da outra, então $\Sigma_{1}=\Sigma_{2}$ em algum aberto em torno de $p$.

(b)(ponto no bordo) Sejam $\Sigma_{1}$ e $\Sigma_{2}$ duas superfícies de tipo espaço com curvatura média constante e bordos diferenciáveis $\partial \Sigma_{1}$ e $\partial \Sigma_{2}$, respectivamente. Assuma que $\Sigma_{1}$ e $\Sigma_{2}$, assim como os seus bordos, são tangentes em um ponto $p \in \partial \Sigma_{1} \cap \partial \Sigma_{2}$, tais que suas curvaturas médias coincidem para uma mesma orientação em p. Se uma delas está localmente acima da outra, então $\Sigma_{1}=\Sigma_{2}$ num aberto com $p$ no seu bordo.

\section{Prova:}

(a) Sejam $U$ um aberto de $T_{p} \Sigma_{1}=T_{p} \Sigma_{2}, u, v: U \rightarrow \mathbb{R}, u=u\left(x_{1}, x_{2}\right), v=v\left(x_{1}, x_{2}\right)$, duas funções diferenciáveis, pelo menos de classe $C^{2}$, contínuas no bordo $\partial U$, cujos gráficos são vizinhanças de $\Sigma_{1}$ e $\Sigma_{2}$, respectivamente, em torno de $p$, com $|D u|<1$ e $|D v|<1$ e $\left(x_{1}, x_{2}\right)$ coordenadas locais em $U$. Como $\Sigma_{1}$ e $\Sigma_{2}$ possuem a mesma curvatura média para alguma orientação comum em $p$, temos que $u$ e $v$ satisfazem a mesma equação diferencial parcial elíptica dada por (3.2) e, portanto, como já vimos, $u-v$ satisfaz uma equação elíptica linear. Além disto, vamos supor que $u \leq v$ em $U$ e então de $u(p)=v(p)$, temos que 0 é o máximo da função $u-v$ em $U, L(u-v)=0$, onde $L$ é um operador linear e $L$ é uniformemente elíptico num subdomínio limitado $U^{\prime} \subset \subset U$ em torno de $p$. Pelo Princípio do Máximo no interior (teorema 3.4.1 (a)) temos que $(u-v)(x)=(u-v)(p)=0 \forall x \in U^{\prime}$. Portanto $u \equiv v$ em $U^{\prime}$ e assim $\Sigma_{1}=\Sigma_{2}$ em alguma vizinhança aberta em torno de $p$.

(b) O raciocínio desta demonstração é bastante parecido ao de (a). A principal diferença é que as funções $u, v$ são definidas num conjunto $D$ que é a união de um conjunto aberto $U$ de $T_{p} \Sigma_{1}=T_{p} \Sigma_{2}$ com uma curva $\Gamma$ contida no bordo de $U$. $\mathrm{O}$ ponto $p \in \Gamma$. A hipótese sobre a tangência também nos bordos implica, além de $(u-v)\left(x_{0}\right)=0$, que a derivada de $u-v$ na direção normal ao bordo de $D$, em $p$, é zero. Deste modo, o resultado segue do Princípio do Máximo no bordo.

Como conseqüência segue 
Corolário 3.6.3. Uma superfície (conexa) compacta máxima $(H=0)$ com bordo planar é uma parte de um plano.

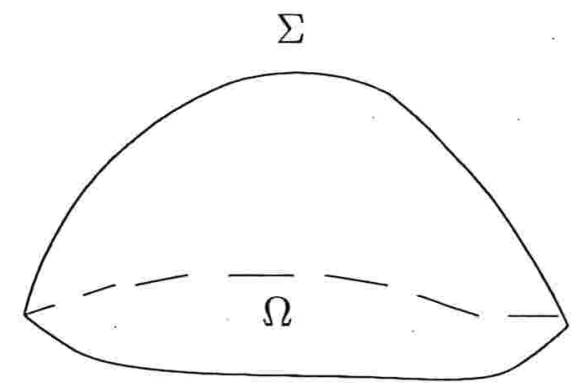

Figura 3.8: Superfície compacta máxima com bordo planar

Prova: É fácil ver que localmente a superfície é parte de um plano, pelo teorema 3.4.1. Agora seja $\Omega$ o domínio limitado pelo bordo da superfície e suponha que existam pontos dela fora de $\Omega$. Então o ponto mais alto (ou o ponto mais baixo) da superfície em relação ao plano contendo o bordo da superfície está fora de $\Omega$. Considere o plano que contém este ponto $p$ que seja paralelo ao plano do bordo. Então este plạno e a superfície coincidem, são tangentes e têm mesma curvatura média $H=0$ neste ponto. Do lema 3.6.2, temos que a superfície coincide localmente com esse plano, em torno deste ponto. Sendo a superfície conexa esse plano deve ser o plano do bordo. 


\section{Capítulo 4}

\section{Unicidade para bordo não conexo}

Neste capítulo, estudamos um resultado de unicidade no caso em que o bordo da superfície não é conexo, através do Método de Reflexão de Alexandrov e do Princípio do Máximo já visto no Capítulo 3.

\subsection{O Método de Reflexão de Alexandrov}

Seja $x: \Sigma \rightarrow \mathbb{L}^{3}$ uma imersão de tipo espaço de uma superfície compacta limitada por duas curvas de Jordan planas $\Gamma_{1}$ e $\Gamma_{2}$, que estão contidas em planos de tipo espaço paralelos $\Pi_{1}$ e $\Pi_{2}$, respectivamente. Podemos assumir, sem perda de generalidade, que $\Pi_{1}$ é o plano $\left\{x_{3}=0\right\}$ e $\Pi_{2}$ é o plano $\left\{x_{3}=c\right\}, c \neq 0$. Seja $\pi: \mathbb{L}^{3} \rightarrow \mathbb{R}^{2}$ a projeção definida por $\pi\left(x_{1}, x_{2}, x_{3}\right)=\left(x_{1}, x_{2}, 0\right)$.

Observação 4.1.1. O fato da imersão ser de tipo espaço impõe restrições sobre as curvas do bordo. Por exemplo, $\pi\left(\Gamma_{2}\right)=\Gamma_{1}$ não pode ocorrer.

De fato, suponhamos $\pi\left(\Gamma_{2}\right)=\Gamma_{1}$ e seja $\pi \circ x: \Sigma \rightarrow \bar{\Omega}$ onde $\Omega=\pi \circ x(\operatorname{int}(\Sigma))$ é o domínio limitado por $\Gamma_{1}$ em $\Pi_{1}$. Com o raciocínio da Proposição 3.1.2, vemos que $\pi \circ x$ é um difeomorfismo, o que é impossível pelo fato de $\pi\left(\Gamma_{1}\right)=\Gamma_{1}=\pi\left(\Gamma_{2}\right)(\pi \circ x$ não é injetora).

Vamos considerar até o fim deste capítulo a situação em que $\pi\left(\Gamma_{2}\right) \subset \Omega_{1}$, onde $\Omega_{1}$ é o domínio limitado por $\Gamma_{1}$. 
Lema 4.1.2. Sejam $\Gamma_{1}$ e $\Gamma_{2}$ duas curvas de Jordan planas contidas nos planos de tipo espaço paralelos $\Pi_{1}=\left\{x_{3}=0\right\}$ e $\Pi_{2}=\left\{x_{3}=c, c \neq 0\right\}$, com $\pi\left(\Gamma_{2}\right) \subset \Omega_{1}$, onde $\Omega_{1}$ é o domínio em $\Pi_{1}$ limitado por $\Gamma_{1}$. Neste caso, é possível mostrar que a projeção da superfície no plano $\Pi_{1}$ é o anel determinado por $\Gamma_{1}$ e $\pi\left(\Gamma_{2}\right)$.

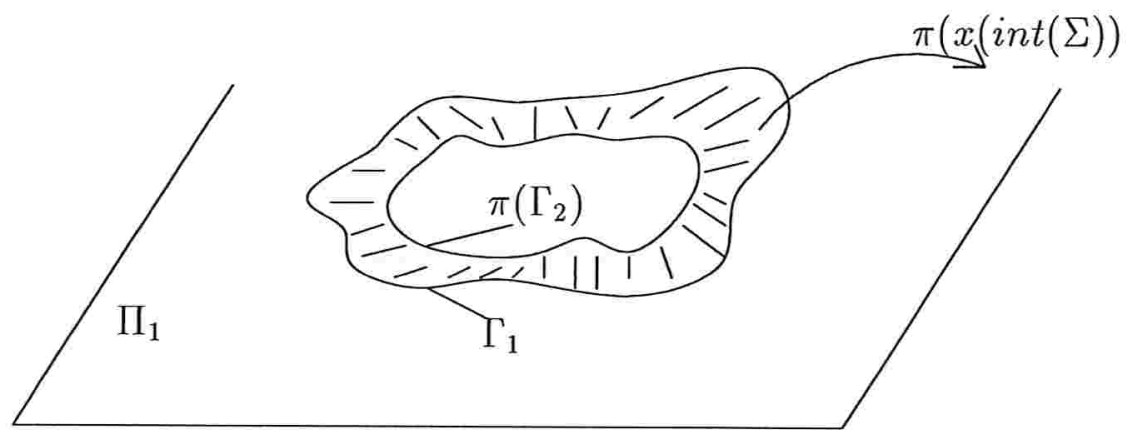

Figura 4.1: Anel determinado por $\Gamma_{1}$ e $\pi\left(\Gamma_{2}\right)$

Prova: Seja $\Omega=\pi \circ x(\operatorname{int}(\Sigma))$ e sejam $\Omega_{1}$ e $\Omega_{2}$ os domínios planares limitados por $\Gamma_{1}$ e $\pi\left(\Gamma_{2}\right)$, respectivamente, com $\pi\left(\Gamma_{2}\right) \subset \Omega_{1}$. Vamos mostrar que $\Omega=\Omega^{\prime}$ onde $\Omega^{\prime}$ é o interior do anel determinado por $\Gamma_{1}$ e $\pi\left(\Gamma_{2}\right)$.

Primeiro mostremos que $\partial \Omega \subset \Gamma_{1} \cup \pi\left(\Gamma_{2}\right)$. Como $\Sigma$ é compacta, para cada $q \in \partial((\pi \circ x)(\Sigma))$ existe $p \in \Sigma$ tal que $\pi \circ x(p)=q$. Vamos mostrar que $p \in \partial \Sigma$. Suponha que $p \in \operatorname{int}(\Sigma)$, então existe uma vizinhança aberta $U_{p}$ de $p$ no $\operatorname{int}(\Sigma)$ e uma vizinhança aberta $V_{q}$ de $q$ em $\Omega$ tal que $\pi \circ x: U_{p} \rightarrow V_{q}$ é um difeomorfismo (pois $\pi \circ x: \operatorname{int}(\Sigma) \rightarrow \mathbb{R}^{2}$ é um difeomorfismo local). Mas isso implica que $q \in \Omega$, o que é um absurdo. Portanto, se $p \in \partial \Sigma$, então $q \in \Gamma_{1}$ ou $q \in \pi\left(\Gamma_{2}\right)$.

Se existe um ponto em $\Omega$ que não está em $\Omega^{\prime}$, como $\Omega$ é limitado, há pontos em $\partial \Omega$ que não estão em $\Gamma_{1} \cup \pi\left(\Gamma_{2}\right)$, o que não é possível.

Da mesma forma, se existe um ponto em $\Omega^{\prime}$ que não está em $\Omega$, há pontos em $\partial \Omega$ dentro de $\Omega^{\prime}$, o que também não é possível. Assim sendo, $\Omega=\Omega^{\prime}$.

Para a demonstração do próximo teorema, será necessário utilizar o Princípio de Reflexão de Alexandrov. Vamos esboçar a idéia deste princípio e, para maiores detalhes, sugerimos [2] e [3]. 


\section{O Método de Reflexão de Alexandrov}

Sejam $\Sigma$ uma superfície compacta com bordo $\Gamma_{1} \cup \Gamma_{2}$ e $G$ o sólido obtido da união de $\Sigma$ com os domínios planares de $\Gamma_{1}$ e $\Gamma_{2}$ contidos em $\Pi_{1}$ e $\Pi_{2}$, respectivamente.

O Método de Alexandrov consiste dos seguintes passos:

1. Tome uma reta $l$ num dos planos $\Pi_{i}$ e desenhe um plano suporte $E$ para $G$ perpendicular a $l$.

2. Mova este plano até que ele comece a atravessar $G$, mantendo sua perpendicularidade a $l$. Assim temos um plano variável perpendicular a $l$.

3. Para cada posição do plano $E$, após seu primeiro contato com $\Sigma$, ele determina em $G$ um novo sólido menor $\mathcal{H}$ que aumenta à medida que $E$ se move.

4. Reflita $\mathcal{H}$ através do plano $E$ obtendo, do outro lado do plano, o sólido refletido $\overline{\mathcal{H}}$. Para pequenos deslocamentos do plano da sua posição inicial, o sólido refletido $\overline{\mathcal{H}}$ está claramente contido em $G$.

5. Em algum momento, a superfície refletida $\bar{\Sigma}$ do sólido $\overline{\mathcal{H}}$ toca a superfície $\Sigma$ do outro lado do plano $E$. Isso se deve ao fato de $\Sigma$ ser compacta.

Na nossa situação, não vamos considerar o sólido $G$, mas apenas o domínio limitado pela junção da superfície com os planos do bordo, que é o interior de $G$. Com estes dados, vamos provar o seguinte teorema que será importante para o próximo resultado de unicidade.

Teorema 4.1.3. Sejam $\Gamma_{1}$ e $\Gamma_{2}$ duas curvas de Jordan convexas contidas nos planos $\Pi_{1}$ e $\Pi_{2}$. Vamos assumir que existe um plano $P$ vertical de simetria de $\Gamma_{1} \cup \Gamma_{2}$. Se $\Sigma e ́$ uma superfície compacta de tipo espaço com curvatura média constante, mergulhada no $\mathbb{L}^{3}$, com bordo $\Gamma_{1} \cup \Gamma_{2}$ e contida na região determinada pelos planos $\Pi_{1}$ e $\Pi_{2}$, então $P$ é um plano de simetria de $\Sigma$.

Prova: Inicialmente vamos anexar a $\Sigma$ os domínios planares limitados por $\Gamma_{1} \mathrm{e}$ $\Gamma_{2}$. Como a superfície está na região determinada pelos planos do bordo, obtemos um domínio limitado $W$ no $\mathbb{L}^{3}$. Agora podemos aplicar o Método de Reflexão de 
Alexandrov, considerando, para simplificar a notação, o plano $P$ como sendo o plano $\left\{x_{2}=0\right\}$ e os planos que se movem paralelamente a $P$, vindos do infinito, como sendo os planos $P_{t}=\left\{x_{2}=t\right\}, t \in \mathbb{R}$. Note que, apesar do Princípio de Reflexão ter sido escrito no espaço Euclideano, nada impede que ele seja aplicado aqui no nosso contexto, já que da forma que a superfície e o plano são tomados, tudo funciona como se estivéssemos refletindo no Euclideano, pois, além da métrica na superfície ser definida positiva, a reflexão em relação a planos perpendiculares ao eixo $O x_{2}$ significa apenas mudança na segunda coordenada dos pontos da superfície.

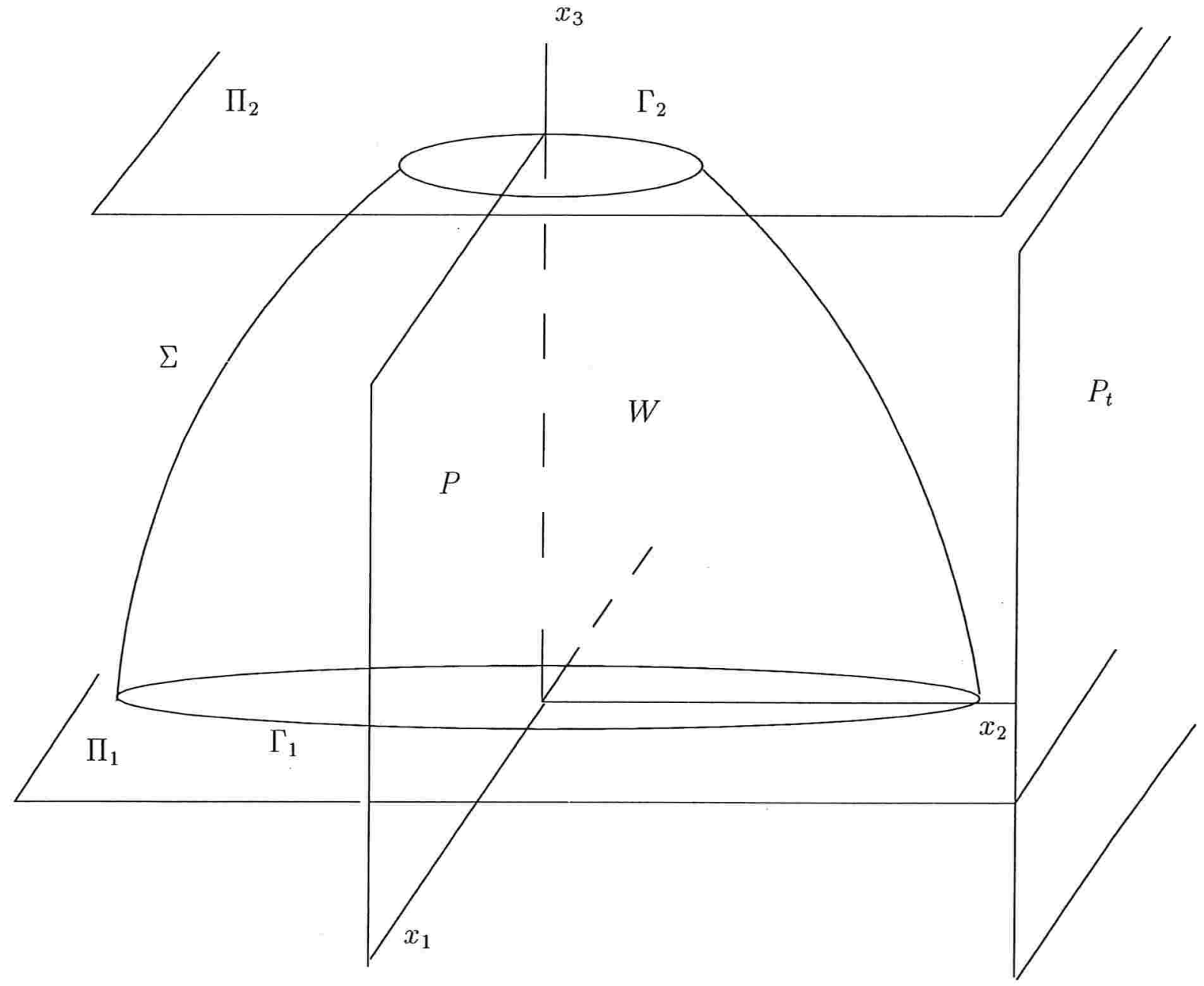

Figura 4.2: Superfície com bordo $\Gamma_{1} \cup \Gamma_{2}$

Tomamos $t>0$ suficientemente grande de modo que o plano $P_{t}$ ainda não intersecte a superfície $\Sigma$. Ao movermos $P_{t}$ para a esquerda, decrescendo $t$, denotamos 
por $P_{t_{0}} \cdot$ o plano que intersecta $\Sigma$ pela primeira vez. Após isso ocorrer, deslocamos levemente $P_{t_{0}}$ para a esquerda, sempre paralelamente a $P$, até obtermos o plano $P_{t}, t_{0}-\varepsilon<t<t_{0}$ que atravessa $W$. Essa parte de $W$ pode consistir de vários pedaços conexos, o que não interfere no processo. Para $\varepsilon$ suficientemente pequeno, a superfície refletida em relação ao plano $P_{t}$, fica contida no conjunto limitado $W$.

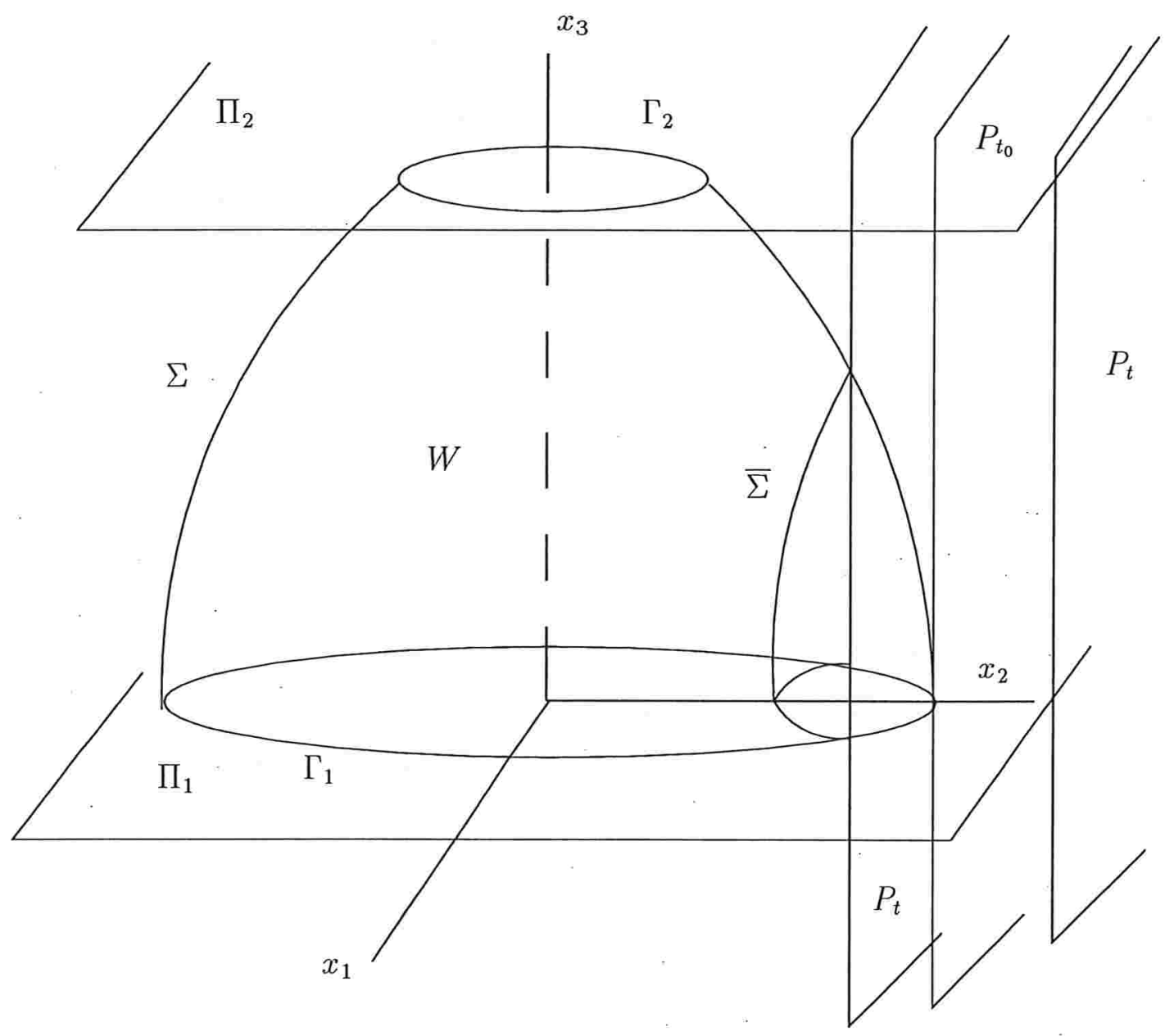

Figura 4.3: Método de reflexão de Alexandrov para $\Sigma$

Se continuarmos movendo $P_{t}$ para a esquerda, decrescendo $t$, então, do fato de $\Sigma$ ser compacta, deve existir um $t_{1}$, que é o maior valor tal que a superfície refletida $\bar{\Sigma}$ através de $P_{t_{1}}$ toca $\Sigma$ do outro lado deste plano num ponto $p$. Observe que $t_{1} \geq 0$ pois $t=0$ corresponde ao plano $P$, que é o plano de simetria do bordo.

Uma vez que os planos $P_{t}$ são perpendiculares aos planos do bordo, há apenas 
duas possibilidades para o ponto $p: p \in \operatorname{int}(\Sigma) \cap \operatorname{int}(\bar{\Sigma})$ ou $p \in \partial \Sigma \cap \partial \bar{\Sigma}$.

No primeiro caso, definimos sobre um domínio $\Omega \subset P_{t_{1}}$ funções diferenciáveis $u, \bar{u}: \Omega \rightarrow \mathbb{R}$ que determinam gráficos de tipo espaço, que são vizinhanças abertas de $\Sigma$ e $\bar{\Sigma}$, respectivamente, em torno do ponto $p$. No ponto de interseção, $u\left(x_{0}\right)=$ $\bar{u}\left(x_{0}\right)=p$ para algum $x_{0} \in \Omega$ e $\Sigma$ está acima de $\bar{\Sigma}$, pois estamos supondo que $p$ é o primeiro ponto de interseção entre as superfícies. Logo $x_{0}$ é o ponto de máximo da função $\bar{u}-u$ e 0 é o máximo desta mesma função. Como as superfícies têm a mesma curvatura média (pois as reflexões com respeito a planos não degenerados são conguências no $\mathbb{L}^{3}$ ) e mesma orientação em $p$, já que a superfície é mergulhada, e portanto, mantém a orientação ao ser refletida, segue que $u$ e $\bar{u}$ satisfazem a equação quasilinear e elíptica (3.6) e, conseqüentemente, $\bar{u}-u$ satisfaz uma equação elíptica linear. Pelo teorema 3.4.1 (a), conclui-se que $u=\bar{u}$ em $\Omega$, isto é, $\Sigma=\bar{\Sigma}$ numa vizinhança do ponto $p$.

Além disto, toda componente conexa $\Sigma^{*}$ de $\bar{\Sigma}$, contendo o ponto $p$, está contida inteiramente em $\Sigma$. De fato, seja $E$ o conjunto de pontos comuns das superfícies $\Sigma$ e $\Sigma^{*}$, isto é, $E=\Sigma^{*} \cap \Sigma$. Mostremos que $E$ é aberto e fechado de $\Sigma^{*}$.

Para provarmos que $E$ é aberto de $\Sigma^{*}$, devemos provar que para cada ponto $q \in E$ fixado arbitrariamente, existe uma vizinhança aberta $U_{q}$, interseção de um aberto $V_{q}$ do $\mathbb{R}^{3}$ com $\Sigma^{*}$, vizinhança de $q$, que esteja totalmente contida em $E$. Temos duas situações para analisar: se $q \in \operatorname{int}(E)$, basta tomarmos $V_{q}$ como sendo int $(E)$ e assim $U_{q}=\operatorname{int}(E) \cap \Sigma^{*}\left(U_{q} \subset E\right.$ pois $\left.\operatorname{int}(E) \subset E \subset \Sigma^{*}\right)$; se $q \in \partial E$, como $\partial E$ é fechado, existe uma vizinhança $V$ de $q$ no $\mathbb{R}^{3}$ tal que $V \cap E \neq \emptyset$ e $V \cap \operatorname{complementar}(E) \neq \emptyset$. Além disto, pelo Princípio do Máximo, supondo $q$ o primeiro ponto de interseção entre $\Sigma$ e $\Sigma^{*}$ nessa vizinhança, temos que existem vizinhanças $\tilde{V}_{q}$ no $\mathbb{R}^{3}$ e $U_{q}$ de $q$ tal que $U_{q}=\tilde{V}_{q} \cap \Sigma^{*}$ onde $\Sigma=\Sigma^{*}$. Pela definição de $E, U_{q} \subset E$. Portanto $E$ é aberto de $\Sigma^{*}$ para todo $q \in E$.

Para provarmos que $E$ é fechado de $\Sigma^{*}$, basta observar que, do que foi feito anteriormente, se $q \in \partial E$ então existe uma vizinhança $U_{q}$ de $q$ tal que $U_{q} \subset E$, isto é, $q \in \operatorname{int}(E)$. Segue que $E$ é fechado de $\Sigma^{*}$.

Note que $E \neq \emptyset$, pois o ponto $p$ encontrado na interseção de $\Sigma$ e $\Sigma^{*}$ pertence a $E$. Logo, sendo aberto, fechado e não vazio, $E \equiv \Sigma^{*}$, com $\Sigma^{*}$ uma componente conexa 
de $\bar{\Sigma}$ contendo $p$. Mas $E=\Sigma \cap \Sigma^{*}$ e, portanto, $\Sigma^{*} \cdot \subset \Sigma$. Segue que as componentes conexas de $\bar{\Sigma}$ e a parte de $\Sigma$ localizada do lado oposto a $\bar{\Sigma}$ (com relação ao plano $\left.P_{t_{1}}\right)$ constituem toda a superfície $\Sigma$.

Por outro lado, $\bar{\Sigma}$ origina-se da reflexão de $\Sigma$ através do plano $P_{t_{1}}$, ou seja, $\Sigma$ é simétrica em relação a esse plano. Logo $P_{t_{1}}$ é um plano de simetria de $\Sigma$ e portanto do seu bordo. Conclui-se, desta forma, que $P_{t_{1}}=P$ e $t_{1}=0$.

Para resolvermos o caso em que $p \in \partial \Sigma \cap \partial \bar{\Sigma}$, vamos fazer com que ele recaia no primeiro caso já analisado.

A primeira observação que fazemos é que o ponto $p \in \Gamma_{1} \cup \Gamma_{2}$. O fato dessas curvas do bordo serem convexas implica que a imagem de $\Gamma_{i}$ pela reflexão coincide com a parte de $\Gamma_{i}$ que está contida do seu mesmo lado, em relação ao plano $P_{t_{1}}$. Mas $P$ é plano de simetria do bordo, portanto $t_{1}=0$. Se repetirmos o mesmo processo de reflexão, agora começando da esquerda para a direita, ou seja, com planos $P_{t}, t<0$, vamos obter da interseção de $\Sigma \operatorname{com} \Sigma \bar{\Sigma}$, um ponto interior $\tilde{p}$ das superfícies, isto é, $\tilde{p} \in \operatorname{int}(\Sigma) \cap \operatorname{int}(\bar{\Sigma})$. Isso ocorre porque se $p \in \partial \Sigma \cap \partial \bar{\Sigma}$, então há pontos do interior de $\Sigma$ que ainda não foram atigindos pela superfície refletida $\bar{\Sigma}$ e são esses pontos que serão refletidos nesta nova situação. Aplicando aqui o mesmo raciocínio utilizado no primeiro caso concluímos que $P$ é um plano de simetria de $\Sigma$.

Até o fim deste capítulo, vamos buscar um resultado de unicidade para as superfícies de revolução do $\mathbb{L}^{3}$ (daí a importância da simetria da superfície). Na verdade, vamos provar que, sob algumas condições envolvendo o seu bordo, uma superfície compacta de tipo espaço com curvatura média constante nula é uma superfície de revolução. A teoria de superfícies de revolução no $\mathbb{L}^{3}$ envolve uma série de conceitos, entre eles os de isometrias e o grupo de Lorentz que veremos agora.

\subsection{Isometrias}

Definição 4.2.1. Uma isometria de $\Sigma$ em $\Sigma$ é um difeomorfismo $\phi: \Sigma \rightarrow \Sigma$ que preserva tensores métricos, isto é,

$$
\left\langle d \phi_{p}(v), d \phi_{p}(w)\right\rangle=\langle v, w\rangle \forall v, w \in T_{p} \Sigma, p \in \Sigma .
$$


Observação 4.2.2. Uma vez que $\phi$ é um difeomorfismo, cada aplicação d $\phi_{p}$ é um isomorfismo linear.

Observação 4.2.3. O conjunto $I(\Sigma)$ de todas as isometrias de $\Sigma$ em $\Sigma$ forma um grupo com a aplicação de composição.

Seja $T: \mathbb{L}^{3} \rightarrow \mathbb{L}^{3}$ um operador linear. Identificamos $T$ com uma matriz $\left(a_{i j}\right), i, j=$ $1, \ldots, 3$, relativamente a uma base ortonormal fixada $\left\{e_{i}\right\}, i=1, \ldots, 3$ onde $a_{i j}=$ $\left\langle T\left(e_{i}\right), T\left(e_{j}\right)\right\rangle$. Com esta identificação a composição de funçốes coincide com a multiplicação de matrizes. Assim, o grupo de transformações lineares do $\mathbb{R}^{3}, G L(3, \mathbb{R})$ fica sendo visto como o grupo de matrizes invertíveis $3 \times 3$ reais. E o conjunto de todas as isometrias lineares $\mathbb{L}^{3} \rightarrow \mathbb{L}^{3}$ fica sendo o conjunto $\mathcal{O}_{1}(3)$ de todas as matrizes $T \in G L(3, \mathbb{R})$ que preservam o produto escalar de $\mathbb{L}^{3}$.

Definição 4.2.4. $\mathcal{O}_{1}(3)=\left\{T \in G L(3, \mathbb{R}):\langle T u, T v\rangle=\langle u, v\rangle \forall u, v \in \mathbb{L}^{3}\right\}$ é o grupo de Lorentz.

Lema 4.2.5. São equivalentes:

1. $T \in O_{1}(3)$

2. $T^{t}=\varepsilon T^{-1} \varepsilon$, onde $\varepsilon=\left(\begin{array}{ccc}1 & 0 & 0 \\ 0 & 1 & 0 \\ 0 & 0 & -1\end{array}\right)$ e $T^{t}$ é a matriz transposta de $T$

3. as colunas (linhas) de $T$ formam uma base ortonormal para $\mathbb{L}^{3}$

4. T leva base ortonormal de $\mathbb{L}^{3}$ em base ortonormal

Observação 4.2.6. $\mathcal{O}_{1}(3)$ tem quatro componentes.

Para vermos isso, escrevemos $M \in \mathcal{O}_{1}(3)$ em forma de uma matriz $\left(\begin{array}{cc}M_{S} & P \\ Q & M_{T}\end{array}\right)$ sendo $M_{T}$ de ordem 1 e $M_{S}$ de ordem 2 .

Aqui a parte de tipo tempo $M_{T}: \mathbb{R}_{1}^{1} \rightarrow \mathbb{R}_{1}^{1}$ é a projeção ortogonal de $M$ restrita a $\mathbb{R}_{1}^{1}$ sobre $\mathbb{R}_{1}^{3}$, onde $\mathbb{L}^{3}=\mathbb{R}_{1}^{3}=\mathbb{R}_{1}^{2} \times \mathbb{R}_{1}^{1}$. Analogamente para a parte de tipo espaço $M_{S}$.

Como $M$ é invertível e preserva caráter causal (pois é isometria), segue que $M_{T}$ e $M_{S}$ são invertíveis. 
Definição 4.2.7: Um elemento $M \in \mathcal{O}_{1}(3)$ preserva [inverte] orientação de tipo tempo se $\operatorname{det} M_{T}>0[<0]$ e preserva [inverte] orientação de tipo espaço se $\operatorname{det} M_{S}>$ $0[<0]$.

Assim $O_{1}(3)$ é decomposto em quatro conjuntos disjuntos indexados pelos sinais $\operatorname{de} \operatorname{det} M_{T}$ e $\operatorname{det} M_{S}$ (nessa ordem):

$$
\mathcal{O}_{1}(3)^{++} \quad \mathcal{O}_{1}(3)^{+-} \quad \mathcal{O}_{1}(3)^{-+} \quad \mathcal{O}_{1}(3)^{--}
$$

Observação 4.2.8. Todo elemento de $\mathcal{O}_{1}(3)$ tem determinante igual $a \pm 1$.

De fato, se $T \in \mathcal{O}_{1}(3)$, pelo lema 4.2 .5 segue que $T^{t}=\varepsilon T^{-1} \varepsilon$.

Tomando o determinante nessa igualdade, temos

$$
\operatorname{det}\left(T^{t}\right)=\operatorname{det}\left(T^{-1}\right)
$$

Mas $\operatorname{det}\left(T^{t}\right)=\operatorname{det}(T)$ e $\operatorname{det}\left(T^{-1}\right)=\frac{1}{\operatorname{det}(T)}$. Logo

$$
(\operatorname{det}(T))^{2}=1 \Rightarrow \operatorname{det}(T)= \pm 1 \text {. }
$$

Definição 4.2.9. O grupo especial de Lorentz é o grupo

$$
\mathcal{S O}_{1}(3)=\left\{T \in \mathcal{O}_{1}(3): \operatorname{det} T=1\right\}
$$

Agora podemos falar das superfícies de revolução de tipo espaço no $\mathbb{L}^{3}$.

\subsection{Superfícies de revolução de tipo espaço}

Seja $l$ uma reta contida num plano П. Denotamos por $\mathcal{O}(l)=\left\{T \in \mathcal{S O}_{1}(3)\right.$ : $T(v)=v, \forall v \in l\}$, ou seja, $\mathcal{O}(l)$ é o conjunto das transformações ortogonais com determinante positivo que deixam $l$ fixada.

Definição 4.3.1. Um círculo em $\mathbb{L}^{3}$ é a órbita de um ponto que está fora de uma reta pela ação do grupo $\mathcal{O}(l)$. 
Desta forma, um círculo está contido num plano ortogonal a $l$. Além disto, os círculos em $\mathbb{L}^{3}$ são descritos a partir do caráter causal da reta $l$.

Observação 4.3.2. A definição do caráter causal de uma reta l no $\mathbb{L}^{3}$ segue a linha da definição para vetores vista na seção 1.1 .

Para conhecermos a forma de uma matriz $T \in \mathcal{O}(l)$, devemos estudar três situações:

1. $l$ é de tipo espaço

2. $l$ é de tipo tempo

3. $l$ é de tipo luz

No caso em que $l$ é de tipo tempo, o grupo $\mathcal{O}(l)$ é formado pelas matrizes $R_{\theta}$ da forma

$$
R_{\theta}=\left(\begin{array}{ccc}
\cos \theta & \sin \theta & 0 \\
-\sin \theta & \cos \theta & 0 \\
0 & 0 & 1
\end{array}\right)
$$

com respeito a uma base ortonormal $\left\{e_{1}, e_{2}, e_{3}\right\}$, onde $e_{3}$ gera a reta $l$ e $\theta \in \mathbb{R}$.

Os círculos de $\mathbb{L}^{3}$ correspondentes a este caso são escritos como

$$
\alpha(s)=c+r\left(\cos (s) e_{1}+\sin (s) e_{2}\right)
$$

$\operatorname{com} r \neq 0, c \in l$.

Observemos que este grupo de rotações $O(l)$ no $\mathbb{L}^{3}$ é o grupo de rotações que fixam $l$ no espaço Euclideano $\mathbb{R}^{3}$.

Definição 4.3.3. Uma superfície de revolução de tipo espaço $\Sigma$ no $\mathbb{L}^{3}$ é o conjunto que se obtém da ação do grupo $\mathcal{O}(l)$ sobre uma curva $\Gamma$ contida num plano $\Pi$ do $\mathbb{L}^{3}$, onde $\Gamma \cap l=\emptyset, l \subset \Pi$, quando este é uma superfície de tipo espaço, isto é,

$$
\Sigma=\{T(p): p \in \Gamma \text { e } T \in \mathcal{O}(l)\}
$$

Isso equivale a dizer que $\Sigma$ é invariante pela ação dos grupos de rotação que deixam $l$ fixa ponto a ponto. 


\subsection{O terceiro resultado de unicidade}

O terceiro e último resultado de unicidade que estudamos é apenas uma consequência do teorema 4.1.3. Mas é preciso que façamos algumas observações antes.

Observação 4.4.1. Numa superfície máxima $(H=0)$, a curvatura média não depende de orientação, portanto, a hipótese mergulho no teorema 4.1.3 pode ser enfraquecida para imersão.

Observação 4.4.2. Toda superfície máxima limitada por duas curvas de Jordan convexas contidas em planos de tipo espaço paralelos está necessariamente contida na região determinada pelos planos do bordo.

De fato, denotemos por $\Sigma$ a superfície em questão e suponhamos que existam pontos de $\Sigma$ fora da região determinada pelos planos do bordo. Então, o ponto mais alto (ou o ponto mais baixo) $p$ da superfície com relação aos planos do bordo não seria um ponto de bordo (já que o bordo está contido nessa região e a superfície é limitada pelas curvas do bordo). Considere o plano que passa por $p$ e é paralelo aos planos do bordo. $\Sigma$ é então tangente ao plano que passa por $p$ e tem a mesma curvatura média que ele. Pelo Princípio da Tangência (Lema 3.6.2), a superfície coincide com o plano numa vizinhança deste ponto, o que é uma contradição.

O corolário seguinte é um resultado de unicidade para as superfícies compactas de tipo espaço imersas no $\mathbb{L}^{3}$ com curvatura média zero.

Corolário 4.4.3. Se $\Sigma$ é uma superfície compacta de tipo espaço imersa no $\mathbb{L}^{3}$ máxima, isto é, com $H=0$, limitada por dois círculos concêntricos contidos em planos paralelos, então $\Sigma$ é uma superfície de revolução.

Prova: Note que os planos $\Pi_{1}$ e $\Pi_{2}$, que contêm os círculos, são de tipo espaço, pois essas curvas são fechadas. Tome $P$ um plano contendo a reta $l$ que une os centros dos círculos que constituem o bordo de $\Sigma$. Tomada desta forma, $l$ é perpendicular aos planos $\Pi_{1}$ e $\Pi_{2}$ e, conseqüentemente, $l$ é de tipo tempo. Após uma isometria do ambiente, podemos assumir que $l$ é o eixo $O x_{3}$. Os círculos que limitam a superfície são, então, círculos Euclideanos em planos paralelos ao plano $\left\{x_{3}=0\right\}$. Logo $P$ é um 
plano vertical de simetria do bordo de $\Sigma$. Pelas observações 4.4 .1 e 4.4.2, podemos aplicar o teorema 4.1.3, concluindo que $P$ é um plano de simetria de $\Sigma$. Como $\Sigma$ é simétrica em relação a $P$, e $P$ foi escolhido arbitrariamente, segue que $\Sigma$ é uma superfície de revolução no $\mathbb{R}^{3}$, ou seja, $\Sigma$ é invariante pela ação do grupo $\mathcal{O}(l)$ descrito em (4.1). Portanto $\Sigma$ é uma superfície de revolução no $\mathbb{L}^{3}$. 


\section{Capítulo 5}

\section{A generalização do primeiro resultado de unicidade}

Neste capítulo, dedicamo-nos à generalização do primeiro resultado de unicidade visto no Capítulo 2. Nossa análise agora é feita para hipersuperfícies de tipo espaço.

A abordagem aqui também é baseada em fórmulas integrais: uma fórmula do fluxo e uma desigualdade integral.

\subsection{Hipersuperfícies compactas de tipo espaço}

Seja $\mathbb{L}^{n+1}=\left(\mathbb{R}^{n+1},\langle\rangle,\right)$ o espaço de Lorentz-Minkowski de dimensão $(n+1)$, onde $\langle\rangle=,\left(d x_{1}\right)^{2}+\ldots+\left(d x_{n}\right)^{2}-\left(d x_{n+1}\right)^{2}$ é a métrica de Lorentz.

Definição 5.1.1. Uma hipersuperfície de tipo espaço imersa no $\mathbb{L}^{n+1}$ é uma variedade diferenciável de dimensão $n$ tal que a métrica induzida $\langle$,$\rangle pela métrica de$ Lorentz é Riemanniana.

Lema 5.1.2. Não existem hipersuperfícies de tipo espaço fechadas no $\mathbb{L}^{n+1}$.

Prova: Seja $M$ uma hiperfsuperfície de tipo espaço no $\mathbb{L}^{n+1}$ e consideremos a função altura $h=\langle a, x\rangle$ definida em $M$, com $a \in \mathbb{L}^{n+1}$ um vetor fixado arbitrariamente. Calculemos $\nabla h$. 


$$
\begin{gathered}
\langle\nabla\langle a, x\rangle, X\rangle=X(\langle a, x\rangle)=\left\langle\nabla_{X}^{\circ} a, x\right\rangle+\left\langle a, \nabla_{X}^{\circ} x\right\rangle=\langle a, X\rangle=\left\langle a^{T}, X\right\rangle \forall X \in \mathcal{X}(\Sigma) \\
\therefore \nabla\langle a, x\rangle=a^{T}=a+\langle a, N\rangle N
\end{gathered}
$$

pois $\nabla_{X}^{\circ} x=\frac{d}{d t}(x \circ \gamma)_{\mid t=0}=\frac{d}{d t}(\gamma)_{\mid t=0}=\gamma^{\prime}(0)=X$ onde $\gamma$ é uma curva em $M$ tal que $\gamma(0)=x$ e $\gamma^{\prime}(0)=X$.

$$
|\nabla\langle a, x\rangle|^{2}=\langle a, a\rangle+\langle a, N\rangle^{2} \geq\langle a, a\rangle .
$$

Logo $h$ não tem pontos críticos quando $a$ é de tipo espaço e, portanto, não tem mínimo nem máximo, conseqüentemente $M$ não pode ser fechada.

Portanto, as hipersuperfícies compactas de tipo espaço têm necessariamente bordo não-vazio.

Denotamos por $M$ a hipersuperfície (conexa) e $\partial M$ o bordo de $M$. Podemos definir o bordo de uma hipersuperfície de tipo espaço imersa no $\mathbb{L}^{n+1}$.

Definição 5.1.3. Se $\Sigma$ é uma (n-1)-subvariedade fechada no $\mathbb{L}^{n+1}$, uma hipersuperfície de tipo espaço $x: M \rightarrow \mathbb{L}^{n+1}$ é dita com bordo $\Sigma$ se a restrição de $x$ ao $\partial M$ é um difeomorfismo sobre $\Sigma$.

Definição 5.1 .4 (Orientação de $\partial M$ ). Uma base $v_{1}, \ldots, v_{n-1}$ para $T_{p}(\partial M)$ está orientada positivamente se e somente se $\left\{v_{1}, \ldots, v_{n-1}, u\right\}$ é uma base orientada positivamente para $T_{p} M$ sempre que $u \in T_{p} M$ está apontando para dentro de $M$ ao longo de $\partial M$.

Denotaremos por $\nu$ o vetor unitário co-normal ao longo de $\partial M$ que aponta para dentro de $M$.

Observação 5.1.5. Denotando por $\nabla^{\circ}$ a conexão Levi-Civita do $\mathbb{L}^{n+1}, \nabla$ a conexão Levi-Civita de $M, A$ o operador de Weingarten associado ao campo normal $N$ unitário, de tipo tempo, apontando para o futuro no $\mathbb{L}^{n+1}$, as equações fundamentais para hipersuperfícies são as mesmas apresentadas no Capítulo 1.(1.1, 1.2, 1.3) 


\subsection{Fórmula do Fluxo}

Seja $a \in \mathbb{L}^{n+1}$ fixado arbitrariamente e $H=-\frac{1}{n} \operatorname{traço}(A)$ a função curvatura de $M$. É facil verificar que $\operatorname{div}\left(a^{T}\right)=n H\langle a, N\rangle$ e do teorema da divergência, segue:

$$
n \int_{M} H\langle N, a\rangle d V=-\oint_{\partial M}\langle a, \nu\rangle d S,
$$

onde $d V$ é o elemento de volume $n$-dimensional de $M$ com respeito à métrica induzida e à orientação escolhida e $d S$ é o elemento de área $(n-1)$-dimensional de $\partial M$.

Consideramos agora a $(n-1)$-forma diferencial $\Theta_{a}$ definida em $M$ por

$$
\Theta_{a}\left(X_{1}, \ldots, X_{n-1}\right)=\operatorname{det}\left(x, X_{1}, \ldots, X_{n-1}, a\right)
$$

para $X_{1}, \ldots, X_{n-1} \in \mathcal{X}(M)$, onde det representa o determinante em $\mathbb{R}^{n+1}$.

Então, de 1.2.9, 1.2.10 e (1.1), segue:

$$
\begin{aligned}
\left(\nabla \Theta_{a}\right)\left(X_{1}, \ldots, X_{n-1}, Y\right)= & \left(\nabla_{Y} \Theta_{a}\right)\left(X_{1}, \ldots, X_{n-1}\right)=Y\left(\Theta_{a}\left(X_{1}, \ldots, X_{n-1}\right)\right)+ \\
& -\sum_{i=1}^{n-1} \Theta_{a}\left(X_{1}, \ldots, X_{i-1}, \nabla_{Y} X_{i}, X_{i+1}, \ldots, X_{n-1}\right)= \\
= & Y\left(\operatorname{det}\left(x, X_{1}, \ldots, X_{n-1}, a\right)\right)+ \\
& -\sum_{i=1}^{n-1} \Theta_{a}\left(X_{1}, \ldots, X_{i-1}, \nabla_{Y}^{\circ} X_{i}, X_{i+1}, \ldots, X_{n-1}\right)+ \\
& -\sum_{i=1}^{n-1} \Theta_{a}\left(X_{1}, \ldots, X_{i-1},\left\langle A Y, X_{i}\right\rangle N, X_{i+1}, \ldots, X_{n-1}\right)= \\
= & \left(\nabla_{Y}^{\circ} \operatorname{det}\right)\left(x, X_{1}, \ldots, X_{n-1}, a\right)+\operatorname{det}\left(\nabla_{Y}^{\circ} x, X_{1}, \ldots, X_{n-1}, a\right)+ \\
& +\sum_{i=1}^{n-1} \operatorname{det}\left(x, X_{1}, \ldots, X_{i-1}, \nabla_{Y}^{\circ} X_{i}, X_{i+1}, \ldots, X_{n-1}, a\right)+ \\
& -\sum_{i=1}^{n-1} \operatorname{det}\left(x, X_{1}, \ldots, X_{i-1}, \nabla_{Y}^{\circ} X_{i}, X_{i+1}, \ldots, X_{n-1}, a\right)+
\end{aligned}
$$




$$
\begin{aligned}
& -\sum_{i=1}^{n-1}\left\langle A X_{i}, Y\right\rangle \Theta_{a}\left(X_{1}, \ldots, X_{i-1}, N, X_{i+1}, \ldots, X_{n-1}\right)= \\
= & \operatorname{det}\left(Y, X_{1}, \ldots, X_{n-1}, a\right)+ \\
& -\sum_{i=1}^{n-1}\left\langle A X_{i}, Y\right\rangle \operatorname{det}\left(x, X_{1}, \ldots, X_{i-1}, N, X_{i+1}, \ldots, X_{n-1}, a\right)
\end{aligned}
$$

para $Y \in \mathcal{X}(M)$, pois note que $\nabla_{Y}^{\circ}($ det $)=0$ (derivada de uma $(n+1)$-forma no $\left.\mathbb{R}^{n+1}\right)$ e a terceira e quarta parcelas cancelam-se.

Portanto, a derivada exterior de $\Theta_{a}$ é dada por:

$$
\begin{aligned}
d \Theta_{a}\left(X_{1}, \ldots, X_{n}\right)= & \sum_{i=1}^{n}(-1)^{i-1}\left(\nabla_{X_{i}} \Theta_{a}\right)\left(X_{1}, \ldots, \hat{X}_{i}, \ldots, X_{n}\right)=\left(\nabla_{X_{1}} \Theta_{a}\right)\left(X_{2}, \ldots, X_{n}\right)- \\
& -\left(\nabla_{X_{2}} \Theta_{a}\right)\left(X_{1}, X_{3}, \ldots, X_{n}\right)+\left(\nabla_{X_{3}} \Theta_{a}\right)\left(X_{1}, X_{2}, X_{4}, \ldots, X_{n}\right)+\ldots+ \\
& +(-1)^{n-1}\left(\nabla_{X_{n}} \Theta_{a}\right)\left(X_{1}, X_{2}, \ldots, X_{n-1}\right)= \\
= & \operatorname{det}\left(X_{1}, X_{2}, \ldots, X_{n}, a\right)-\left\langle A X_{2}, X_{1}\right\rangle \operatorname{det}\left(x, N, X_{3}, \ldots, X_{n}, a\right)+ \\
& -\left\langle A X_{3}, X_{1}\right\rangle \operatorname{det}\left(x, X_{2}, N, X_{4}, \ldots, X_{n}, a\right)+\ldots+ \\
& -\left\langle A X_{n}, X_{1}\right\rangle \operatorname{det}\left(x, X_{2}, \ldots, X_{n-1}, N, a\right)-\operatorname{det}\left(X_{2}, X_{1}, X_{3}, \ldots, X_{n}, a\right)+ \\
& +\left\langle A X_{1}, X_{2}\right\rangle \operatorname{det}\left(x, N, X_{3}, \ldots, X_{n}, a\right)+ \\
& +\left\langle A X_{3}, X_{2}\right\rangle \operatorname{det}\left(x, X_{1}, N, X_{4}, \ldots, X_{n}, a\right)+\ldots+ \\
& +\left\langle A X_{n}, X_{2}\right\rangle \operatorname{det}\left(x, X_{1}, X_{3}, \ldots, X_{n-1}, N, a\right)+ \\
& +\operatorname{det}\left(X_{3}, X_{1}, X_{2}, X_{4}, \ldots, X_{n}, a\right)-\left\langle A X_{1}, X_{3}\right\rangle \operatorname{det}\left(x, N, X_{2}, X_{4}, \ldots, X_{n}, a\right)+ \\
& -\left\langle A X_{2}, X_{3}\right\rangle \operatorname{det}\left(x, X_{1}, N, X_{4}, \ldots, X_{n}, a\right)-\ldots- \\
& -\left\langle A X_{n}, X_{3}\right\rangle \operatorname{det}\left(x, X_{1}, X_{2}, X_{4}, \ldots, X_{n-1}, N, a\right)+\ldots+
\end{aligned}
$$




$$
\begin{aligned}
& +(-1)^{n-1}\left[\operatorname{det}\left(X_{n}, X_{1}, \ldots, X_{n-1}, a\right)-\left\langle A X_{1}, X_{n}\right\rangle \operatorname{det}\left(x, N, X_{2}, \ldots, X_{n-1}, a\right)\right. \\
& -\left\langle A X_{2}, X_{n}\right\rangle \operatorname{det}\left(x, X_{1}, N, X_{3}, \ldots, X_{n-1}, a\right)-\ldots- \\
& \left.-\left\langle A X_{n-1}, X_{n}\right\rangle \operatorname{det}\left(x, X_{1}, \ldots, X_{n-2}, N, a\right)\right]= \\
& =n \operatorname{det}\left(X_{1}, X_{2}, \ldots, X_{n}, a\right)^{1}
\end{aligned}
$$

Por outro lado,

$$
\begin{aligned}
\operatorname{det}\left(X_{1}, X_{2}, \ldots, X_{n}, a\right) & =\left\langle X_{1} \wedge X_{2} \wedge \ldots \wedge X_{n}, a\right\rangle= \\
& =-\left\langle d V\left(X_{1}, \ldots, X_{n}\right) N, a\right\rangle= \\
& =-\langle N, a\rangle d V\left(X_{1}, \ldots, X_{n}\right),
\end{aligned}
$$

pois $d V\left(X_{1}, \ldots, X_{n}\right)=\left\langle N, X_{1} \wedge X_{2}, \ldots, X_{n}\right\rangle$.

Ou seja $d \Theta_{a}=-n\langle N, a\rangle d V$.

Se a curvatura média $H$ é constante, a equação acima nos dá a seguinte fórmula do fluxo:

Lema 5.2.1 (Fórmula do fluxo). Seja $x: M \rightarrow \mathbb{L}^{n+1}$ uma imersão de tipo espaço de uma hipersuperfície compacta com bordo $\partial M$. Se a curvatura média $H$ é constante, então para qualquer vetor fixado $a \in \mathbb{L}^{n+1}$ temos

$$
\oint_{\partial M}\langle\nu, a\rangle d S=H \oint_{\partial M} \Theta_{a}
$$

onde $\nu$ é o vetor unitário co-normal apontando para dentro ao longo do bordo e $\Theta_{a}$ é dado por (5.2).

Prova: De (5.1) e (5.3)

$$
\begin{gathered}
\oint_{\partial M}\langle\nu, a\rangle d S=-n \int_{M} H\langle N, a\rangle d V= \\
=H \int_{M}-n\langle N, a\rangle d V=H \int_{M} d \Theta_{a}=H \oint_{\partial M} \Theta_{a}
\end{gathered}
$$

\footnotetext{
${ }^{1}$ observe que as parcelas $\left\langle A X_{i}, X_{j}\right\rangle \operatorname{det}(\ldots)$ cancelam-se com as parcelas $\left\langle A X_{j}, X_{i}\right\rangle \operatorname{det}(\ldots)$.
} 


\subsection{Conseqüências da fórmula do fluxo para bordo hiperplanar}

Assumimos que $\Sigma=x(\partial M)$ está contido num hiperplano fixado $\Pi$ do $\mathbb{L}^{n+1}$.

Lema 5.3.1. O hiperplano $\Pi$ é de tipo espaço.

Prova: Suponhamos que $\Pi$ é hiperplano passando pela origem e seja $a \in \mathbb{L}^{n+1}$ o vetor normal a $\Pi$. Consideremos a função altura $h=\langle a, x\rangle$ definida em $\partial M$. Tal como no lema 5.1.2, é fácil verificar que quando $a$ é de tipo espaço $h$ não tem pontos críticos. Isso implica que $\Sigma$ não é fechada, o que é uma contradição, pois $x$ restrita a $\partial M$ é um difeomorfismo e $\partial M$ é fechado. Portanto, o vetor normal ao plano II é de tipo tempo. Pelo Lema 1.1.5, considerando $\Pi=a^{\perp}$, temos que $\Pi$ é de tipo espaço.

Seja, então, $a \in \mathbb{L}^{n+1}$ um vetor de tipo tempo unitário que aponta para o futuro tal que $\Pi=a^{\perp}$. Uma aplicação da fórmula do fluxo para este caso é dada abaixo:

Proposição 5.3.2. Seja $x: M \rightarrow \mathbb{L}^{n+1}$ uma imersão de tipo espaço de uma hipersuperfície compacta limitada por uma subvariedade (n-1)-dimensional $\Sigma=x(\partial M)$ mergulhada e assuma que $\Sigma$ está contida num hiperplano $\Pi=a^{\perp}$ como acima descrito. Se a curvatura média $H$ de $M$ é constante, então

$$
\oint_{\partial M}\langle\nu, a\rangle d S=n H \operatorname{vol}(\Omega)
$$

onde $\Omega$ é o domínio em $\Pi$ limitado por $\Sigma$.

Prova: Do Lema 5.2.1

$$
\oint_{\partial M}\langle\nu, a\rangle d S=H \oint_{\partial M} \Theta_{a} .
$$

Logo vemos que basta mostrarmos que

$$
\oint_{\partial M} \Theta_{a}=n \operatorname{vol}(\Omega)
$$

A primeira informação que temos é que $\Sigma$ é uma subvariedade fechada e mergulhada no $\mathbb{I}^{n+1}$ contida em $\Pi$. Este é o mesmo papel que uma curva de Jordan plana tem na dimensão 2. Ou seja, vale a seguinte expressão para o volume:

$$
\operatorname{vol}(\Omega)=-\frac{1}{n} \oint_{\partial M}\langle x, \eta\rangle d S
$$


onde $\eta$ é o normal unitário que aponta para dentro de $\Sigma$ em $\Pi$.

Consideramos a orientação natural em $\Pi$ determinada por $a$, isto é, $\left\{e_{1}, \ldots, e_{n-1}, \eta\right\}$ é uma base para $\Pi$, onde $\left\{e_{1}, \ldots, e_{n-1}\right\}$ é um referencial ortonormal (definido localmente) orientado positivamente tangente ao longo de $\partial M$. Então $\left\{e_{1}, \ldots, e_{n-1}, \eta, a\right\}$ está orientado positivamente e $\operatorname{det}\left(e_{1}, \ldots, e_{n-1}, \eta, a\right)=1$. Usando (5.2) isso implica que

$$
\begin{aligned}
\Theta_{a}\left(e_{1}, \ldots, e_{n-1}\right) & =\operatorname{det}\left(x, e_{1}, \ldots, e_{n-1}, a\right)=\left\langle x, e_{1} \wedge \ldots \wedge e_{n-1} \wedge a\right\rangle= \\
= & -\left\langle x, a \wedge e_{1} \wedge \ldots \wedge e_{n-1}\right\rangle=-\langle x, \eta\rangle
\end{aligned}
$$

e de (5.5) e (5.6) segue que

$$
\oint_{\partial M} \Theta_{a}=-\oint_{\partial M}\langle x, \eta\rangle d S=n \operatorname{vol}(\Omega)
$$

\subsection{Uma desigualdade integral}

Queremos calcular agora $\operatorname{div}\left(A\left(a^{T}\right)+H a^{T}\right)$.

Cálculos análogos aos do Capítulo 2 mostram que

$$
\operatorname{div}\left(A\left(a^{T}\right)\right)=-n\langle\nabla H, a\rangle-\langle a, N\rangle \operatorname{traço}\left(A^{2}\right) .
$$

Portanto se $H$ é constante, temos

$$
\operatorname{div}\left(A\left(a^{T}\right)+H a^{T}\right)=-\langle a, N\rangle\left(n^{2} H^{2}+n K-n H^{2}\right) .
$$

Aplicando o teorema da divergência, obtemos o seguinte resultado:

Proposição 5.4.1. Seja $x: M \rightarrow \mathbb{L}^{n+1}$ uma imersão de tipo espaço de uma hipersuperfície compacta limitada por uma (n-1)-subvariedade mergulhada $\Sigma=x(\partial M) e$ assuma que $\Sigma$ está contida no hiperplano $\Pi=a^{\perp}$, com a um vetor unitário de tipo tempo apontando para o futuro no $\mathbb{L}^{n+1}$.

Se a curvatura média $H$ de $M$ é constante, então

$$
\oint_{\partial M} H_{\Sigma}\langle\nu, a\rangle^{2} d S \leq n H^{2} \operatorname{vol}(\Omega)
$$


onde $\Omega$ é o domínio em $\Pi$ limitado por $\Sigma$ e $H_{\Sigma}$ a curvatura média de $\Sigma$ em $\Pi$ com respeito ao normal unitário $\eta$ que aponta para dentro de $\Sigma$.

Além disto, a igualdade vale se e somente se a hipersuperfície é totalmente umbílica.

Prova: Integrando (5.7) sobre $M$ e usando o teorema da divergência

$$
\begin{gathered}
\int_{M} \operatorname{div}\left(A\left(a^{T}\right)+H a^{T}\right) d V=-\int_{M}\langle a, N\rangle\left(n^{2} H^{2}+n K-n H^{2}\right) d V= \\
=-\oint_{\partial M}\left\langle A\left(a^{T}\right), \nu\right\rangle d S-H \oint_{\partial M}\left\langle a^{T}, \nu\right\rangle d S=-\oint_{\partial M}(H\langle\nu, a\rangle+\langle A(\nu), a\rangle) d S
\end{gathered}
$$

Sabemos que $\langle a, N\rangle \leq-1$, pois $N$ e a pertencem ao mesmo cone de tipo tempo e que $n^{2} H^{2}+n K-n H^{2}=\operatorname{traço}\left(A^{2}\right)-\frac{1}{n}(\operatorname{traço}(A))^{2} \geq 0$.

Portanto

$$
\oint_{\partial M}(H\langle\nu, a\rangle+\langle A(\nu), a\rangle) d S \leq 0,
$$

e a igualdade vale se e somente se a hipersuperfície é totalmente umbílica.

Vamos calcular $\langle A(\nu), a\rangle$.

Escrevemos $a$ na base $\left\{e_{1}, \ldots, e_{n-1}, \nu, N\right\}$, onde $\left\{e_{1}, \ldots, e_{n-1}\right\}$ é um referencial local ortonormal tangente orientado positivamente ao longo de $\partial M$. Uma vez que $\left\langle a, e_{i}\right\rangle=$ 0 , ficamos com

$$
a=\langle a, \nu\rangle \nu-\langle a, N\rangle N .
$$

Logo, $\langle A(\nu), a\rangle=\langle a, \nu\rangle\langle A(\nu), \nu\rangle$.

Por outro lado, $\left\{e_{1}, \ldots, e_{n-1}, \nu\right\}$ é um referencial local ortonormal para $T_{p} M$, portanto

$$
-n H=\sum_{i=1}^{n-1}\left\langle A\left(e_{i}\right), e_{i}\right\rangle+\langle A(\nu), \nu\rangle
$$

onde $\operatorname{traço}(A)=-n H$. De forma que

$$
\langle A(\nu), a\rangle=\langle a, \nu\rangle\left(-n H-\sum_{i=1}^{n-1}\left\langle A\left(e_{i}\right), e_{i}\right\rangle\right) .
$$

Precisamos encontrar $\left\langle A\left(e_{i}\right), e_{i}\right\rangle$. Seja $A_{\Sigma}$ o operador de Weingarten associado a $\eta$. Vamos escrever $\nabla_{e_{i}}^{\circ} e_{i}$ nas bases do $\mathbb{L}^{n+1}:\left\{e_{1}, \ldots, e_{n-1}, \nu, N\right\}$ e $\left\{e_{1}, \ldots, e_{n-1}, \eta, a\right\}$ 
$\operatorname{De}\left\langle e_{i}, e_{i}\right\rangle=1$ temos $\left\langle\nabla_{e_{i}}^{\circ} e_{i}, e_{i}\right\rangle=0$ e segue

$$
\begin{aligned}
\nabla_{e_{i}}^{\circ} e_{i} & =\sum_{i \neq j}\left\langle\nabla_{e_{i}}^{\circ} e_{i}, e_{j}\right\rangle e_{j}+\left\langle\nabla_{e_{i}}^{\circ} e_{i}, \nu\right\rangle \nu-\left\langle\nabla_{e_{i}}^{\circ} e_{i}, N\right\rangle N \\
& =\sum_{i \neq j}\left\langle\nabla_{e_{i}}^{\circ} e_{i}, e_{j}\right\rangle e_{j}+\left\langle\nabla_{e_{i}}^{\circ} e_{i}, \nu\right\rangle \nu-\left\langle A\left(e_{i}\right), e_{i}\right\rangle N
\end{aligned}
$$

$\operatorname{De}\left\langle e_{i}, a\right\rangle=0$ temos $\left\langle\nabla_{e_{i}}^{\circ} e_{i}, a\right\rangle=0$ e segue

$$
\nabla_{e_{i}}^{\circ} e_{i}=\sum_{i \neq j}\left\langle\nabla_{e_{i}}^{\circ} e_{i}, e_{j}\right\rangle e_{j}+\left\langle\nabla_{e_{i}}^{\circ} e_{i}, \eta\right\rangle \eta=\sum_{i \neq j}\left\langle\nabla_{e_{i}}^{\circ} e_{i}, e_{j}\right\rangle e_{j}+\left\langle A_{\Sigma}\left(e_{i}\right), e_{i}\right\rangle \eta .
$$

Essas igualdades implicam que

$$
\begin{gathered}
\left\langle\nabla_{e_{i}}^{\circ} e_{i}, \nu\right\rangle \nu-\left\langle A\left(e_{i}\right), e_{i}\right\rangle N=\left\langle A_{\Sigma}\left(e_{i}\right), e_{i}\right\rangle \eta \\
\therefore-\left\langle A\left(e_{i}\right), e_{i}\right\rangle N=\left\langle A_{\Sigma}\left(e_{i}\right), e_{i}\right\rangle \eta-\left\langle\nabla_{e_{i}}^{\circ} e_{i}, \nu\right\rangle \nu \\
\therefore\left\langle A\left(e_{i}\right), e_{i}\right\rangle=\left\langle A_{\Sigma}\left(e_{i}\right), e_{i}\right\rangle\langle\eta, N\rangle \\
\therefore\left\langle A\left(e_{i}\right), e_{i}\right\rangle=-\left\langle A_{\Sigma}\left(e_{i}\right), e_{i}\right\rangle\langle\nu, a\rangle
\end{gathered}
$$

pois $\langle\eta ; N\rangle=\left\langle a \wedge e_{1} \wedge \ldots \wedge e_{n-1}, N\right\rangle=-\left\langle a, N \wedge e_{1} \wedge \ldots \wedge e_{n-1}\right\rangle=-\langle a, \nu\rangle$

Portanto

$$
\langle A(\nu), a\rangle=\langle a, \nu\rangle\left(-n H+\sum_{i=1}^{n-1}\left\langle A_{\Sigma}\left(e_{i}\right), e_{i}\right\rangle\langle\nu, a\rangle\right) .
$$

Reescrevendo a desigualdade (5.10) temos

$$
\begin{aligned}
& \oint_{\partial M}\langle\nu, a\rangle\left(H-n H+\sum_{i=1}^{n-1}\left\langle A_{\Sigma}\left(e_{i}\right), e_{i}\right\rangle\langle\nu, a\rangle\right) d S= \\
= & \left.\oint_{\partial M}\langle\nu, a\rangle((1-n) H)+\langle\nu, a\rangle(n-1) H_{\Sigma}\right) d S \leq 0
\end{aligned}
$$

onde $(n-1) H_{\Sigma}=\operatorname{traço}\left(A_{\Sigma}\right)$.

$$
\therefore \oint_{\partial M} H_{\Sigma}\langle\nu, a\rangle d S^{2} \leq \oint_{\partial M} H\langle\nu, a\rangle d S=n H^{2} \operatorname{vol}(\Omega)
$$

o que prova (5.8).

Além disto, a igualdade vale em (5.8) se e somente se a hipersuperfície é totalmente umbílica, de acordo com (5.10). 
Observamos que, analogamente à Proposição 2.7.1, as hipersuperfícies de tipo espaço conexas totalmente umbílicas são os hiperplanos e os hiperbolóides.

Temos, agora, tudo o que é necessário para provarmos o teorema de unicidade.

Teorema 5.4.2. As únicas hipersuperfícies compactas de tipo espaço imersas no $\mathbb{L}^{n+1}$ com curvatura média constante $H$ e bordo esférico são as bolas hiperplanares $(H=0)$ e as calotas hiperbólicas $(H \neq 0)$.

Prova: Se o bordo $\Sigma=x(\partial M)$ é uma esfera $S^{n-1}(r)$ de raio $r>0$, a proposição 5.4.1 e o fato de $H_{\Sigma}=\frac{1}{r}$ implicam que

$$
\oint_{\partial M}\langle\nu, a\rangle^{2} d S \leq n H^{2} \operatorname{vol}\left(B^{n}(r)\right) \cdot r=H^{2} r^{2} \text { área }\left(S^{n-1}(r)\right)
$$

com a igualdade valendo se e somente se $M$ é totalmente umbílica.

Além disto, a proposição 5.3.2 afirma que

$$
\oint_{\partial M}\langle\nu, a\rangle d S=n H \operatorname{vol}\left(B^{n}(r)\right)=H r \text { área }\left(S^{n-1}(r)\right)
$$

e da conseqüência da desigualdade de Hölder obtemos

$$
\begin{gathered}
\oint_{\partial M}\langle\nu, a\rangle^{2} d S \cdot \oint_{\partial M} 1 d S \geq\left(\oint_{\partial M}\langle\nu, a\rangle d S\right)^{2} \\
\therefore \oint_{\partial M}\langle\nu, a\rangle^{2} d S \geq \frac{H^{2} r^{2}\left(\operatorname{área}\left(S^{n-1}(r)\right)\right)^{2}}{\operatorname{ar} r e a\left(S^{n-1}(r)\right)}=H^{2} r^{2} \text { área }\left(S^{n-1}(r)\right) .
\end{gathered}
$$

Logo vale a igualdade, então as hipersuperfícies são totalmente umbílicas. Para ter bordo esférico, elas devem ser discos hiperplanares quando $H=0$ e calotas hiperbólicas quando $H \neq 0$. 


\section{Referências Bibliográficas}

[1] K. Akutagawa, S.Nishikawa, The Gauss map and spacelike surfaces with prescribed mean curvature in Minkowski 3-space, Tôhoku Math. J.42 (1990), 67-82.

[2] A.D. Alexandrov, A characteristic property of spheres, Ann.Mat. Pura Appl.58(1962), 303-315.

[3] A.D. Alexandrov, Uniqueness theorems for surfaces in the large V, AMS Transl, 21 (1962), 412-415.

[4] L.J. Alías, R. López, J.A. Pastor, Compact spacelike surfaces with constant mean curvature in the Lorentz-Minkowski 3-space, Tôhoku Math.J.50(1998) 491-501.

[5] L.J. Alías, J. A. Pastor, Constant mean curvature spacelike hypersurfaces with spherical boundary in the Lorentz-Minkowski space, J. of Geometry and Physics 28(1998), 85-93.

[6] J.L.M. Barbosa, R.S. Earp, Geometric methods and non-linear analysis in Hyperbolic Space, X Escola de Geometria Diferencial, UFMG(1998)

[7] J.L.M. Barbosa, Constant mean curvature surfaces bounded by a plane curve, Matemática Contemporânea, vol.1 (1991), 3-15, SBM.

[8] J.K. Beem, P.E. Ehrlich, K.L. Easley, Global lorentzian geometry, NY, 1996.

[9] F. Brito, R.S. Earp, W.H. Meeks III, H. Rosenberg, Stuctures theorems for constant mean curvature surfaces bounded by a planar curve, Indiana Univ. Math J.40 (1991), 333-343. 
[10] M.P. Carmo, Differential geometry of curves and surfaces, Prentice-Hall, New Jersey, 1976.

[11] P. M. Carrillo, Geometria de las superficies maximales en el espacio de LorentzMinkowski, Tesina de Licenciatura, Universidade de Murcia, Espanha (2000).

[12] D. Gilbarg, N.S. Trudinger, Elliptic partial differential equations of second order, Springer-Verlag, Berlin, 1983.

[13] J. I. Hano, Surfaces of revolution with constant mean curvature in Lorentz Minkowski space, Tôhoku Math J. 36 (1984), 427-437.

[14] C.C. Hsiung, Almost complex and complex structures, Series in Pure Math-V.20, World Scientific(1995).

[15] K. Kodaira, J. Morrow, Complex manifolds, NY, Holt, Rinehart and Winston(1971).

[16] M. Koiso, Symmetry of hypersurfaces of constante mean curvature with symmetric boundary, Math.Z., 191 (1986), 567-754.

[17] K.R.F. Leão, O princípio da tangência e suas aplicações, Tese de Mestrado, IMPA-R.J. 1983, Brasil.

[18] Maria L. Leite, The tangency principle for hypersurfaces with a null intermediate curvature, XI Escola de Geometria Diferencial, Univ. Federal Fluminense, Niterói(2000).

[19] R. López, S. Montiel, Constant mean curvature discs with bounded area, Proc. Amer. Math. Soc. 123 (1995), 1555-1558.

[20] R. López, S. Montiel, Constant mean curvature surfaces with planar boundary, Duke Math, J.85 (1996), 583-604.

[21] R. López, Constant mean curvature surfaces foliated by circles in LorentzMinkowski spaces, Geometriae Dedicata 76 (1999), 81-95.

[22] J. R. Munkres, Analysis on manifolds, Redwood City, Addison-Wesley, 1991. 
[23] B. O'Neill, Semi-riemannian geometry with applications to relativity, NY Academic Press(1983).

[24] T.Y. Wan, Constant mean curvature surfaces, harmonic maps, and universal Teichmüller space, J. Differential Geom. 35 (1992), 643-657. 